\title{
On the structure of framed vertex operator algebras and their pointwise frame stabilizers
}

\author{
Ching Hung Lam* \\ Department of Mathematics, National Cheng Kung University \\ Tainan, Taiwan 701 \\ Hiroshi Yamauchi ${ }^{\dagger}$ \\ Graduate School of Mathematical ciences, the University of Tokyo \\ 3-8-1 Komaba, Meguro-ku, Tokyo 153-8914, Japan \\ 2000 Mathematics Subject Classification. Primary 17B69; Secondary 20B25. \\ Dedicated to Professor Koichiro Harada on his 65th birthday
}

\begin{abstract}
In this paper, we study the structure of a general framed vertex operator algebra (VOA). We show that the structure codes $(C, D)$ of a framed VOA $V$ satisfy certain duality conditions. As a consequence, we prove that every framed VOA is a simple current extension of the associated binary code VOA $V_{C}$. This result suggests the feasibility of classifying framed vertex operator algebras, at least if the central charge is small. In addition, the pointwise frame stabilizer of $V$ is studied. We completely determine all automorphisms in the pointwise stabilizer, which are of order 1, 2 or 4 . The 4A-twisted sector and the 4A-twisted orbifold theory of the famous moonshine VOA $V^{\natural}$ are also constructed explicitly. We verify that the top module of this twisted sector is of dimension 1 and of weight $3 / 4$ and the VOA obtained by 4A-twisted orbifold construction of $V^{\natural}$ is isomorphic to $V^{\natural}$ itself.
\end{abstract}

\footnotetext{
*Partially supported by NSC grant 94-2115-M-006-001 of Taiwan, R.O.C.

†Supported by JSPS Research Fellowships for Young Scientists.
} 


\section{Contents}

1 Introduction

2 Preliminaries on simple current extensions $\quad 7$

2.1 Fusion algebras . . . . . . . . . . . . . . . . . . . . . 7

2.2 Simple current extensions . . . . . . . . . . . . . . . . . . . . . 9

3 Ising frame and framed VOA $\quad 12$

3.1 Miyamoto involutions . . . . . . . . . . . . . . . . 12

3.2 Framed VOAs and their structure codes . . . . . . . . . . . . . 13

4 Representation of code VOAs $\quad 14$

4.1 Central extension of codes . . . . . . . . . . . . . . . . . . 14

4.2 Structure of modules . . . . . . . . . . . . . . . . . . . 16

4.3 Dual module . . . . . . . . . . . . . . . . . . . 21

4.4 Fusion rules . . . . . . . . . . . . . . . . . . . . . . . 23

5 Structure of framed VOAs $\quad 25$

5.1 Simple current structure . . . . . . . . . . . . . . . . . 26

5.2 Construction of a framed VOA . . . . . . . . . . . . . 32

6 Frame stabilizers and order four symmetries

7 4A-twisted orbifold construction $\quad 45$ 


\section{Introduction}

A framed vertex operator algebra $V$ is a simple vertex operator algebra (VOA) which contains a sub VOA $F$ called a Virasoro frame isomorphic to a tensor product of $n$ copies of the simple Virasoro VOA $L(1 / 2,0)$ such that the conformal element of $F$ is the same as the conformal element of $V$. There are many important examples such as the moonshine VOA $V^{\natural}$ and the Leech lattice VOA. In [DGH], a basic theory of framed VOAs was established. A general structure theory about the automorphism group and the frame stabilizer, the subgroup which stabilizes $F$ setwise, was also included. Moreover, Miyamoto M3 showed that if $V=\oplus_{n \in \mathbb{Z}} V_{n}$ is a framed VOA over $\mathbb{R}$ such that $V$ has a positive definite invariant bilinear form and $V_{1}=0$, then the full automorphism group $\operatorname{Aut}(V)$ is finite (see also [M1, M2]). Hence, the theory of framed VOA is very useful in studying certain finite groups such as the Monster.

It is well-known (cf. [DMZ, DGH, [M3]) that for any framed VOA $V$ with a frame $F$, one can associate two binary codes $C$ and $D$ to $V$ as follows.

Since $F \simeq L(1 / 2,0)^{\otimes n}$ is a rational vertex operator algebra, $V$ is completely reducible as an $F$-module. That is,

$$
V=\bigoplus_{h_{i} \in\{0,1 / 2,1 / 16\}} m_{h_{1}, \ldots, h_{n}} L\left(1 / 2, h_{1}\right) \otimes \cdots \otimes L\left(1 / 2, h_{n}\right),
$$

where $m_{h_{1}, \ldots, h_{r}}$ is the multiplicity of the $F$-module $L\left(1 / 2, h_{1}\right) \otimes \cdots \otimes L\left(1 / 2, h_{n}\right)$ in $V$. In particular, all the multiplicities are finite and $m_{h_{1}, \ldots, h_{r}}$ is at most 1 if all $h_{i}$ are different from $1 / 16$.

Let $M=L\left(1 / 2, h_{1}\right) \otimes \cdots \otimes L\left(1 / 2, h_{n}\right)$ be an irreducible module over $F$. The $1 / 16$-word (or $\tau$-word) $\tau(M)$ of $M$ is a binary codeword $\beta=\left(\beta_{1}, \ldots, \beta_{n}\right) \in \mathbb{Z}_{2}^{n}$ such that

$$
\beta_{i}= \begin{cases}0 & \text { if } h_{i}=0 \text { or } 1 / 2 \\ 1 & \text { if } h_{i}=1 / 16\end{cases}
$$

For any $\alpha \in \mathbb{Z}_{2}^{n}$, define $V^{\alpha}$ as the sum of all irreducible submodules $M$ of $V$ such that $\tau(M)=\alpha$. Denote $D:=\left\{\alpha \in \mathbb{Z}_{2}^{n} \mid V^{\alpha} \neq 0\right\}$. Then $D$ is an even linear subcode of $\mathbb{Z}_{2}^{n}$ and we obtain a $D$-graded structure on $V=\oplus_{\alpha \in D} V^{\alpha}$ such that $V^{\alpha} \cdot V^{\beta}=V^{\alpha+\beta}$. In particular, $V^{0}$ itself is a subalgebra and $V$ can be viewed as a $D$-graded extension of $V^{0}$.

For any $\gamma=\left(\gamma_{1}, \ldots, \gamma_{n}\right) \in \mathbb{Z}_{2}^{n}$, denote $V(\gamma):=L\left(1 / 2, h_{1}\right) \otimes \cdots \otimes L\left(1 / 2, h_{n}\right)$ where $h_{i}=$ $1 / 2$ if $\gamma_{i}=1$ and $h_{i}=0$ elsewhere. Set

$$
C:=\left\{\gamma \in \mathbb{Z}_{2}^{n} \mid m_{\gamma_{1} / 2, \ldots, \gamma_{n} / 2} \neq 0\right\}
$$

Then $V(0)=F$ and $V^{0}=\oplus_{\gamma \in C} V(\gamma)$. The sub VOA $V^{0}$ forms a $C$-graded simple current extension of $F$ which has a unique simple VOA structure [M2]. A VOA of the form $V^{0}=\oplus_{\gamma \in C} V(\gamma)$ is often referred to as a code $V O A$ associated to $C$. 
The codes $C$ and $D$ are very important parameters for $V$ and we shall call them the structures codes of $V$ with respect to the frame $F$. One of the main purposes of this paper is to study the precise relations between the structure codes $C$ and $D$. As our main result, we shall show in Theorem 5.5 that for any $\alpha \in D$, the subcode $C_{\alpha}:=\{\beta \in$ $C \mid \operatorname{supp}(\beta) \subset \operatorname{supp}(\alpha)\}$ contains a doubly even subcode which is self-dual with respect to $\alpha$. From this we can prove that every framed VOA forms a $D$-graded simple current extension of a code VOA associated to $C$ in Theorem 5.6. This shows that one can obtain any framed VOA by performing simple current extensions in two steps: first extend $F$ to a code VOA $V_{C}$ associated to $C$, then form a $D$-graded simple current extension of $V_{C}$ by adjoining suitable irreducible $V_{C}$-modules. The structure and representation theory of simple current extensions is well-developed by many authors [DM1, M2, L3, Y1, Y2]. It is known that a simple current extension has a unique structure of a simple vertex operator algebra. Since $F$ is rational, this implies there exist only finitely many inequivalent framed VOAs with a given central charge. Therefore, together with the conditions on $(C, D)$ in Theorem 5.5, our results provide a method for determining all framed VOAs with a fixed central charge, at least if the central charge is small. It is well-known that the structure codes $(C, D)$ of a holomorphic framed VOA must satisfy $C=D^{\perp}$ (cf. [DGH, M3]). In this case, we shall describe some necessary and sufficient conditions which $C$ has to satisfy. Namely, we shall show in Theorem 5.17 that there exists a holomorphic framed VOA with structure codes $\left(C, C^{\perp}\right)$ if and only if $C$ satisfies the following.

(1) The length of $C$ is divisible by 16 .

(2) $C$ is even, every codeword of $C^{\perp}$ has a weight divisible by 8 , and $C^{\perp} \subset C$.

(3) For any $\alpha \in C^{\perp}$, the subcode $C_{\alpha}$ of $C$ contains a doubly even subcode which is self-dual with respect to $\alpha$.

We shall call such a code an F-admissible code.

Since the conditions above provide quite strong restrictions on a code $C$, it is possible to classify all the codes satisfying these conditions if the length is small. Once the classification of the $F$-admissible codes of a fixed length is done, one can consider the classification of holomorphic framed VOAs with the corresponding central charge since a holomorphic framed VOA is always a simple current extension of a code VOA. Based on the results of the present paper, one can also characterize the moonshine vertex operator algebra as the unique holomorphic framed vertex operator algebra of central charge 24 with trivial weight one subspace (cf. [LY], see also Remark [5.18). It is a special case of the famous uniqueness conjecture of Frenkel-Lepowsky-Meurman [FLM].

In our argument, doubly even self-dual codes play an important role in prescribing 
structures of framed VOAs, and it is also revealed that if we omit the doubly even property, then we lose the self-duality of certain summands $V^{\alpha}$ of $V$ which will give rise to an involutive symmetry analogous to the lift of the $(-1)$-isometry on a lattice VOA $V_{L}$. By the standard notation as in [FLM], a lattice VOA has a form

$$
V_{L}=\bigoplus_{\alpha \in L} M_{\mathfrak{h}}(\alpha)
$$

where $M_{\mathfrak{h}}(\alpha)$ denotes the irreducible highest weight representation over the free bosonic vertex operator algebra $M_{\mathfrak{h}}(0)$ associated to the vector space $\mathfrak{h}=\mathbb{C} \otimes_{\mathbb{Z}} L$ with highest weight $\alpha \in \mathfrak{h}^{*}=\mathfrak{h}$. Since the fusion algebra associated to $M_{\mathfrak{h}}(0)$ is canonically isomorphic to the group algebra $\mathbb{C}[\mathfrak{h}]$, one has a duality relation $M_{\mathfrak{h}}(\alpha)^{*} \simeq M_{\mathfrak{h}}(-\alpha)$. This shows that there exists an order two symmetry inside the decomposition (1.2), namely, we can define an involution $\theta \in \operatorname{Aut}\left(V_{L}\right)$ such that $\theta M_{\mathfrak{h}}(\alpha)=M_{\mathfrak{h}}(-\alpha)$ which is an extension of an involution on $M_{\mathfrak{h}}(0)$. However, since a framed VOA $V$ has a decomposition $V=\oplus_{\alpha \in D} V^{\alpha}$ graded by an elementary abelian 2-group $D$, one cannot see the analogous symmetry directly from the decomposition. We shall show that by breaking the doubly even property in $(C, D)$, we can find a pair of structure subcodes $\left(C^{0}, D^{0}\right)$ with $\left[C: C^{0}\right]=\left[D: D^{0}\right]=2$ such that one can obtain a decomposition

$$
V=\left(\bigoplus_{\alpha \in D^{0}} V^{\alpha+} \oplus V^{\alpha-}\right) \bigoplus\left(\bigoplus_{\alpha \in D^{1}} V^{\alpha+} \oplus V^{\alpha-}\right)
$$

which forms a $\left(D^{0} \oplus \mathbb{Z}_{4}\right)$-graded simple current extension of a code VOA associated to $C^{0}$, where $D^{1}$ is the complement of $D^{0}$ in $D$. Actually, the main motivation of the present work is to obtain the decomposition above. In the study of McKay's $E_{8}$-observation on the Monster simple group [LYY1, LYY2], the authors found that McKay's $E_{8}$-observation is related the conjectural $\mathbb{Z}_{p}$-orbifold construction of the moonshine VOA from the Leech lattice VOA for $p>2$, where the case $p=2$ is solved in [FLM, Y3]. Based on the decomposition (1.3), we can perform a $\mathbb{Z}_{4}$-twisted orbifold construction on $V^{\natural}$.

The order four symmetry defined by the decomposition in (1.3) can be found as an automorphism fixing $F$ pointwise. The group of automorphisms which fixes $F$ pointwise is referred to as the pointwise frame stabilizer of $V$. We shall show that the pointwise frame stabilizer only has elements of order 1, 2 or 4 and it is completely determined by the structure codes $(C, D)$. As an example, we compute the pointwise stabilizer of the Moonshine VOA $V^{\natural}$ associated with a frame given in [DGH, M3. A 4A-element of the Monster is described as an element of the pointwise frame stabilizer and the associated McKay-Thompson series is computed in the proof of Theorem [7.5. In addition, the 4Atwisted sector and the 4A-twisted orbifold theory of $V^{\natural}$ are constructed. We shall verify that the lowest degree subspace of this twisted sector is of dimension 1 and of weight $3 / 4$, 
and the VOA obtained by the 4A-twisted orbifold construction of $V^{\natural}$ is isomorphic to $V^{\natural}$ itself.

Acknowledgment The authors thank Masahiko Miyamoto for discussions and valuable comments on the proof of Theorem 5.5. They also thank Masaaki Kitazume and Hiroki Shimakura for discussions on binary codes. The second-named author wishes to thank Markus Rosellen for information on the associativity and the locality of vertex operators. Part of the work was done when the second author was visiting the National Center for Theoretical Sciences, Taiwan on February 2006. He thanks the staff of the center for their hospitality.

Notation and Terminology In this article, $\mathbb{N}, \mathbb{Z}$ and $\mathbb{C}$ denote the set of non-negative integers, integers, and the complex numbers, respectively. For disjoint subsets $A$ and $B$ of a set $X$, we use $A \sqcup B$ to denote the disjoint union. Every vertex operator algebra is defined over the complex number field $\mathbb{C}$ unless otherwise stated. A VOA $V$ is called of CFT-type if it has the grading $V=\oplus_{n \geq 0} V_{n}$ with $V_{0}=\mathbb{C} \mathbb{1}$. For a VOA structure $(V, Y(\cdot, z), \mathbb{1}, \omega)$ on $V$, the vector $\omega$ is called the conformal vecton of $V$. For simplicity, we often use $(V, \omega)$ to denote the structure $(V, Y(\cdot, z), \mathbb{1}, \omega)$. The vertex operator $Y(a, z)$ of $a \in V$ is expanded as $Y(a, z)=\sum_{n \in \mathbb{Z}} a_{(n)} z^{-n-1}$. For subsets $A \subset V$ and $B \subset M$ of a $V$-module $M$, we set

$$
A \cdot B:=\operatorname{Span}_{\mathbb{C}}\left\{a_{(n)} v \mid a \in A, v \in B, n \in \mathbb{Z}\right\}
$$

If $M$ has an $L(0)$-weight space decomposition $M=\oplus_{n=0}^{\infty} M_{n+h}$ with $M_{h} \neq 0$, we call $M_{h}$ the top level or top module of $M$ and $h$ the top weight of $M$. The top level and top weight of a twisted module can be defined similarly.

For $c, h \in \mathbb{C}$, let $L(c, h)$ be the irreducible highest weight module over the Virasoro algebra with central charge $c$ and highest weight $h$. It is well-known that $L(c, 0)$ has a simple VOA structure. An element $e \in V$ is referred to as a Virasoro vector with central charge $c_{e} \in \mathbb{C}$ if $e \in V_{2}$ and it satisfies $e_{(1)} e=2 e$ and $e_{(3)} e=(1 / 2) c_{e} \mathbb{1}$. It is well-known that by setting $L^{e}(n):=e_{(n+1)}, n \in \mathbb{Z}$, we obtain a representation of the Virasoro algebra on $V$ (cf. [M1]), i.e.,

$$
\left[L^{e}(m), L^{e}(n)\right]=(m-n) L^{e}(m+n)+\delta_{m+n, 0} \frac{m^{3}-m}{12} c_{e}
$$

Therefore, a Virasoro vector together with the vacuum element generates a Virasoro VOA inside $V$. We shall denote this subalgebra by $\operatorname{Vir}(e)$.

\footnotetext{
${ }^{1}$ We have changed the definition of the conformal vector and the Virasoro vector. In our past works, their definitions are opposite.
} 
In this paper, we define a sub VOA of $V$ to be a pair $(U, e)$ such that $U$ is a subalgebra of $V$ containing the vacuum element $\mathbb{1}$ and $e$ is the conformal vector of $U$. Note that $(U, e)$ inherits the grading of $V$, that is, $U=\oplus_{n \geq 0} U_{n}$ with $U_{n}=V_{n} \cap U$, but $e$ may not be the conformal vector of $V$. In the case that $e$ is also the conformal vector of $V$, we shall call the sub $\operatorname{VOA}(U, e)$ a full sub $\operatorname{VOA} 2$.

For a positive definite even lattice $L$, we shall denote the lattice VOA associated to $L$ by $V_{L}$ (cf. [FLM]). We adopt the standard notation for $V_{L}$ as in [FLM]. In particular, $V_{L}^{+}$denotes the fixed point subalgebra of $V_{L}$ by a lift of $(-1)$-isometry on $L$. The letter $\Lambda$ always denotes the Leech lattice, the unique even unimodular lattice of rank 24 without roots.

Given an automorphism group $G$ of $V$, we denote by $V^{G}$ the fixed point subalgebra of $G$ in $V$. The subalgebra $V^{G}$ is called the $G$-orbifold of $V$ in the literature. For a $V$-module $\left(M, Y_{M}(\cdot, z)\right)$ and $\sigma \in \operatorname{Aut}(V)$, we set $Y_{M}^{\sigma}(a, z):=Y_{M}(\sigma a, z)$ for $a \in V$. Then the $\sigma$-conjugate module $M^{\sigma}$ of $M$ is defined to be the module $\left(M, Y_{M}^{\sigma}(\cdot, z)\right)$.

We denote the ring $\mathbb{Z} / p \mathbb{Z}$ by $\mathbb{Z}_{p}$ with $p \in \mathbb{Z}$ and often identify the integers $0,1, \ldots, p-1$ with their images in $\mathbb{Z}_{p}$. An additive subgroup $C$ of $\mathbb{Z}_{2}^{n}$ together with the standard $\mathbb{Z}_{2^{-}}$ bilinear form is called a linear code. For a codeword $\alpha=\left(\alpha_{1}, \ldots, \alpha_{n}\right) \in C$, we define the support of $\alpha$ by $\operatorname{supp}(\alpha):=\left\{i \mid \alpha_{i}=1\right\}$ and the weight by $\operatorname{wt}(\alpha):=|\operatorname{supp}(\alpha)|$. For a subset $A$ of $C$, we define $\operatorname{supp}(A):=\cup_{\alpha \in A} \operatorname{supp}(\alpha)$. For a binary codeword $\gamma \in \mathbb{Z}_{2}^{n}$ and for any linear code $C \subset \mathbb{Z}_{2}^{n}$, we denote $C_{\gamma}:=\{\alpha \in C \mid \operatorname{supp}(\alpha) \subset \operatorname{supp}(\gamma)\}$ and $C^{\perp_{\gamma}}:=\left\{\beta \in C^{\perp} \mid \operatorname{supp}(\beta) \subset \operatorname{supp}(\gamma)\right\}$, where $C^{\perp}=\left\{\delta \in \mathbb{Z}_{2}^{n} \mid\langle\alpha, \delta\rangle=0\right.$ for all $\left.\alpha \in \mathbb{C}\right\}$. A subcode $H$ of $C$ is called self-dual with respect to $\beta \in C$ if $\operatorname{supp}(H)=\operatorname{supp}(\beta)$ and $H^{\perp_{\beta}}=H$ (see also Definition 4.16). The all-one vector is a codeword $(11 \ldots 1) \in \mathbb{Z}_{2}^{n}$. For $\alpha=\left(\alpha_{1}, \ldots, \alpha_{n}\right)$ and $\beta \in\left(\beta_{1}, \ldots, \beta_{n}\right) \in \mathbb{Z}_{2}^{n}$, we define

$$
\alpha \cdot \beta:=\left(\alpha_{1} \beta_{1}, \ldots, \alpha_{n} \beta_{n}\right) \in \mathbb{Z}_{2}^{n}
$$

That is, the product $\alpha \cdot \beta$ is taken in the ring $\mathbb{Z}_{2}^{n}$. Note that $\alpha \cdot \beta \in\left(\mathbb{Z}_{2}^{n}\right)_{\alpha} \cap\left(\mathbb{Z}_{2}^{n}\right)_{\beta}$.

\section{Preliminaries on simple current extensions}

We shall present some basic facts on simple current extensions of a rational $C_{2}$-cofinite vertex operator algebra of CFT-type.

\subsection{Fusion algebras}

We recall the notion of the fusion algebra associated to a rational VOA $V$. It is known that a rational VOA $V$ has finitely many inequivalent irreducible modules (cf. [DLM2]).

\footnotetext{
${ }^{2}$ It is also called a conformal sub VOA in the literature.
} 
Let $\operatorname{Irr}(V)=\left\{X^{i} \mid 1 \leq i \leq r\right\}$ be the set of inequivalent irreducible $V$-modules. It is shown in [HL] that the fusion product $X^{i} \otimes_{V} X^{j}$ exists for a rational VOA $V$. The irreducible decomposition of $X^{i} \bigotimes_{V} X^{j}$ is referred to as the fusion rule

$$
X^{i} \underset{V}{\otimes} X^{j}=\bigoplus_{k=1}^{r} N_{i j}^{k} X^{k},
$$

where the integer $N_{i j}^{k} \in \mathbb{Z}$ denotes the multiplicity of $X^{k}$ in the fusion product, and is called the fusion coefficient which is also the dimension of the space of all $V$-intertwining operators of type $X^{i} \times X^{j} \rightarrow X^{k}$. We shall denote by $\left(\begin{array}{c}X^{k} \\ X^{i} X^{j}\end{array}\right)_{V}$ the space of $V$-intertwining operators of type $X^{i} \times X^{j} \rightarrow X^{k}$. We define the fusion algebra (or the Verlinde algebra) associated to $V$ by the linear space $\mathcal{V}(V)=\oplus_{i=1}^{r} \mathbb{C} X^{i}$ spanned by a formal basis $\left\{X^{i} \mid\right.$ $1 \leq i \leq r\}$ equipped with a product defined by the fusion rule (2.1). By the symmetry of fusion coefficients, the fusion algebra $\mathcal{V}(V)$ is commutative (cf. [FHL]). Moreover, it is shown in [H3] that if $V$ is rational, $C_{2}$-cofinite and of CFT-type, then $\mathcal{V}(V)$ is associative. In this subsection, we assume that $V$ is rational, $C_{2}$-cofinite and of CFT-type.

A $V$-module $M$ is called a simple current if for any irreducible $V$-module $X$, the fusion product $M \otimes_{V} X$ is also irreducible. In other words, a simple current $V$-module $M$ induces a permutation on $\operatorname{Irr}(V)$ via $X \mapsto M \otimes_{V} X$ for $X \in \operatorname{Irr}(V)$. Note that $V$ itself is a simple current $V$-module.

Next we shall recall the notion of the dual module. For a graded $V$-module $M=$ $\oplus_{n \in \mathbb{N}} M_{n+h}$ such that $\operatorname{dim} M_{n+h}<\infty$, define its restricted dual by $M^{*}=\oplus_{n \in \mathbb{N}} M_{n+h}^{*}$, where $M_{n+h}^{*}:=\operatorname{Hom}_{\mathbb{C}}\left(M_{n+h}, \mathbb{C}\right)$ is the dual space of $M_{h+n}$. Let $Y_{M}(\cdot, z)$ be the vertex operator on $M$. We can introduce the contragredient vertex operator $Y_{M}^{*}(\cdot, z)$ on $M^{*}$ defined by

$$
\left\langle Y_{M}^{*}(a, z) x, v\right\rangle:=\left\langle x, Y_{M}\left(e^{z L(1)}\left(-z^{-2}\right)^{L(0)} a, z^{-1}\right) v\right\rangle
$$

for $a \in V, x \in M^{*}$ and $v \in M$ (cf. [FHL]). The module $\left(M^{*}, Y_{M}^{*}(\cdot, z)\right)$ is called the dual (or contragredient) module of $M$.

Note that if the dual module $M^{*}$ of $M$ is isomorphic to $N$, there exists a $V$-isomorphism $f \in \operatorname{Hom}_{V}\left(N, M^{*}\right)$. Then $f$ induces a $V$-intertwining operator of type $V \times N \rightarrow M^{*}$. This implies that $\left(\begin{array}{c}V^{*} \\ M\end{array}\right)_{V} \neq 0$ or equivalently $M \bigotimes_{V} N \supset V^{*}$. A $V$-module $M$ is called self-dual if $M^{*} \simeq M$. It is obvious that the space of $V$-invariant bilinear forms on an irreducible self-dual $V$-module is one-dimensional.

Lemma 2.1. ([YQ]) Let $U, W$ be $V$-modules such that $U \otimes_{V} W=V$ in the fusion algebra. Then both $U$ and $W$ are simple current $V$-modules.

Proof: First, we show that $U \bigotimes_{V} M \neq 0$ for any $V$-module $M$. We may assume that $M$ is irreducible as $V$ is rational. Since the fusion product is commutative and associative, we have $\left(U \bigotimes_{V} M\right) \bigotimes_{V} W=\left(U \bigotimes_{V} W\right) \bigotimes_{V} M=V \bigotimes_{V} M=M$. This shows that $U \bigotimes_{V} M \neq$ 
0. Similarly, $W \bigotimes_{V} M \neq 0$. Now assume that $U \bigotimes_{V} M=M^{1} \oplus M^{2}$ for $V$-submodules $M^{1}$ and $M^{2}$. Then $W \bigotimes_{V}\left(U \bigotimes_{V} M\right)=\left(W \bigotimes_{V} M^{1}\right) \oplus\left(W \bigotimes_{V} M^{2}\right)$. On the other hand,

$$
W \underset{V}{\underset{V}{\otimes}}(U \underset{V}{\otimes} M)=(W \underset{V}{\underset{V}{\otimes}} U) \underset{V}{\underset{\nabla}{\otimes}} M=V \underset{V}{\otimes} M=M
$$

Therefore, $\left(W \bigotimes_{V} M^{1}\right) \oplus\left(W \bigotimes_{V} M^{2}\right)=M$. Since $W \bigotimes_{V} M^{i} \neq 0$ if $M^{i} \neq 0$, we see that $U \otimes_{V} M$ is irreducible if $M$ is. This shows that $U$, and also $W$, are simple current $V$-modules.

Corollary 2.2. Assume that $V$ is simple, rational, $C_{2}$-cofinite, of CFT-type and self-dual. Then the following hold.

(1) Every simple current $V$-module is irreducible.

(2) A $V$-module $U$ is simple current if and only if $U \nabla_{V} U^{*}=V$.

(3) The set of simple current $V$-modules forms a multiplicative abelian group in $\mathcal{V}(V)$ under the fusion product.

Proof: Let $U$ be a simple current $V$-module. Then $U=V \bigotimes_{V} U$ is irreducible as $V$ is simple. By the symmetry of fusion rules $\left(\begin{array}{c}U \\ V U\end{array}\right)_{V} \simeq\left(\begin{array}{c}U \\ U V\end{array}\right)_{V} \simeq\left(\begin{array}{c}V^{*} \\ U U^{*}\end{array}\right)_{V}$ (cf. [FHL]) and the assumption $V^{*} \simeq V$, we have $U \bigotimes_{V} U^{*} \supset V$. Since $U$ and $U^{*}$ are irreducible, we have $U \bigotimes_{V} U^{*}=V$. This shows (1) and (2). Now let $\mathcal{A}$ be the subset of $\mathcal{V}(V)$ consisting of all the (inequivalent) simple current $V$-modules. Since a fusion product of simple current modules is again a simple current, $\mathcal{A}$ is closed under the fusion product. Clearly $V \in \mathcal{A}$ so that $\mathcal{A}$ contains a unit element. Finally, if $U \in \mathcal{A}$, then $U \otimes_{V} U^{*}=V$ so that the inverse $U^{*} \in \mathcal{A}$ by (2). This completes the proof.

\subsection{Simple current extensions}

We review some basic results about simple current extensions from [L3, Y1]. Let $V^{0}$ be a simple rational $C_{2}$-cofinite VOA of CFT type and let $\left\{V^{\alpha} \mid \alpha \in D\right\}$ be a set of inequivalent irreducible $V^{0}$-modules indexed by an abelian group $D$. A simple VOA $V_{D}=\oplus_{\alpha \in D} V^{\alpha}$ is called a D-graded extension of $V^{0}$ if $V^{0}$ is a full sub VOA of $V_{D}$ and $V_{D}$ carries a $D$-grading, i.e., $V^{\alpha} \cdot V^{\beta} \subset V^{\alpha+\beta}$ for $\alpha, \beta \in D$. In this case, the dual group $D^{*}$ of $D$ acts naturally and faithfully on $V_{D}$. If all $V^{\alpha}, \alpha \in D$, are simple current $V^{0}$-modules, then $V_{D}$ is referred to as a D-graded simple current extension of $V^{0}$. The abelian group $D$ is automatically finite since $V^{0}$ is rational (cf. [DLM2]).

Proposition 2.3. ([ABD, DM2, [L3, $Y Y 1])$ Let $V^{0}$ be a simple rational $C_{2}$-cofinite VOA of CFT type. Let $V_{D}=\oplus_{\alpha \in D} V^{\alpha}$ be a D-graded simple current extension of $V^{0}$. Then

(1) $V_{D}$ is rational and $C_{2}$-cofinite.

(2) If $\tilde{V}_{D}=\oplus_{\alpha \in D} \tilde{V}^{\alpha}$ is another D-graded simple current extension of $V^{0}$ such that $\tilde{V}^{\alpha} \simeq$ $V^{\alpha}$ as $V^{0}$-modules for all $\alpha \in D$, then $V_{D}$ and $\tilde{V}_{D}$ are isomorphic VOAs over $\mathbb{C}$. 
(3) For any subgroup $E$ of $D$, a subalgebra $V_{E}:=\oplus_{\alpha \in E} V^{\alpha}$ is an E-graded simple current extension of $V^{0}$. Moreover, $V_{D}$ is a $D /$ E-graded simple current extension of $V_{E}$.

A representation theory of simple current extensions is developed in [L3, Y1]. It is shown that each irreducible module over a simple current extension corresponds to an irreducible module over a finite dimensional semisimple associative algebra. Moreover, it is also proved that any $V^{0}$-module can be extended to certain twisted modules over $V_{D}$.

Let $M$ be an irreducible $V_{D}$-module. Since $V^{0}$ is rational, we can take an irreducible $V^{0}$-submodule $W$ of $M$. Define $D_{W}:=\left\{\alpha \in D \mid V^{\alpha} \bigotimes_{V^{0}} W \simeq_{V^{0}} W\right\}$. Then $D_{W}$ is a subgroup of $D$. Note that the subgroup $D_{W}$ is independent of the choice of the irreducible $V^{0}$-module $W$ in $M$. In other words, $D_{W}=D_{W^{\prime}}$ for any irreducible $V^{0}$-submodules $W$ and $W^{\prime}$ of $M$. We call $M \quad D$-stable if $D_{W}=0$. In this case, $V^{\alpha} \otimes_{V^{0}} W \simeq_{V^{0}} V^{\beta} \bigotimes_{V^{0}} W$ if and only if $\alpha=\beta$ and by setting $M^{\alpha}:=V^{\alpha} \bigotimes_{V^{0}} W$, we have a $D$-graded isotypical decomposition $M=\oplus_{\alpha \in D} M^{\alpha}\left(\simeq V \bigotimes_{V^{0}} W\right)$ as a $V^{0}$-module.

Theorem 2.4. ([L3, YY1] ) Let $W$ be an irreducible $V^{0}$-module. Then there exists a unique $\chi_{W} \in D^{*} \subset \operatorname{Aut}\left(V_{D}\right)$ such that $W$ can be extended to an irreducible $\chi_{W}$-twisted $V_{D}$-module. If $D_{W}=0$, then the extension of $W$ to an irreducible $\chi_{W}$-twisted $V_{D}$-module is unique and D-stable. Moreover, the extension of $W$ is given by $V_{D} \bigotimes_{V^{0}} W$ as a $V^{0}$-module.

One can easily compute fusion rules among irreducible $D$-stable modules.

Proposition 2.5. ([SY,,$Y 1]$ ) Let $V_{D}$ be a D-graded simple current extension of a simple rational $C_{2}$-cofinite VOA $V^{0}$ of CFT-type. Let $M^{i}, i=1,2,3$ be irreducible $D$-stable $V_{D^{-}}$ modules. Denote by $M^{i}=\oplus_{\alpha \in D}\left(M^{i}\right)^{\alpha}$ a D-graded isotypical decomposition of $M^{i}$. Then the following linear isomorphism holds:

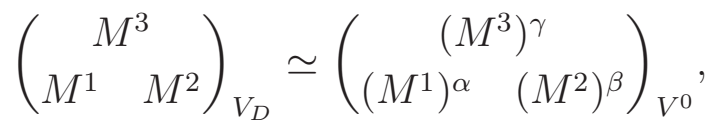

where $\alpha, \beta, \gamma \in D$ are arbitrary.

We shall need the following result on $\mathbb{Z}_{2}$-graded simple current extensions.

Proposition 2.6. Let $V^{0}$ be a simple rational $C_{2}$-cofinite self-dual VOA of CFT-type. Let $V^{1}$ be a simple current $V^{0}$-module not isomorphic to $V^{0}$ such that $V^{1} \bigotimes_{V^{0}} V^{1}=V^{0}$. Assume that $V^{1}$ has an integral top weight and the invariant bilinear form on $V^{1}$ is symmetric. Then there exists a unique simple $V O A$ structure on $V=V^{0} \oplus V^{1}$ as a $\mathbb{Z}_{2}$-graded simple current extension of $V^{0}$.

Proof: For $a, b \in V^{0}$ and $u, v \in V^{1}$, define a vertex operator $Y(\cdot, z)$ as follows:

$$
Y(a, z) b:=Y_{V^{0}}(a, z) b, Y(a, z) u:=Y_{V^{1}}(a, z) u, Y(u, z) a:=e^{z L(-1)} Y(a,-z) u
$$


and $Y(u, z) v$ is defined by means of the matrix coefficients

$$
\langle Y(u, z) v, a\rangle_{V^{0}}=\left\langle v, Y\left(e^{z L(1)}\left(-z^{-2}\right)^{L(0)} u, z^{-1}\right) a\right\rangle_{V^{1}}
$$

where $\langle\cdot, \cdot\rangle_{V^{i}}$ denotes the invariant bilinear form on $V^{i}, i=0,1$. Since the invariant bilinear form on $V^{1}$ is symmetric, we have the skew-symmetry $Y(u, z) v=e^{z L(-1)} Y(v,-z) u$ for any $u, v \in V^{1}$ by Proposition 5.6.1 of [FHL]. It is also shown in [FHL, Li2] that $\left(V^{0} \oplus V^{1}, Y(\cdot, z)\right)$ forms a $\mathbb{Z}_{2}$-graded simple vertex operator algebra if and only if we have a locality for any three elements in $V^{1}$, that is, for any $u, v \in V^{1}$, there exists $N \in \mathbb{N}$ such that for any $w \in V^{1}$ we have

$$
\left(z_{1}-z_{2}\right)^{N} Y\left(u, z_{1}\right) Y\left(v, z_{2}\right) w=\left(z_{1}-z_{2}\right)^{N} Y\left(v, z_{2}\right) Y\left(u, z_{1}\right) w .
$$

By Huang [H3, Theorem 3.5] (see also Theorem 3.2 and 3.5 of [H1]), it is shown that there exists $\lambda \in \mathbb{C}^{*}$ such that for any $u, v, w \in V^{1}$ and sufficiently large $k \in \mathbb{Z}$, we have

$$
\left(z_{0}+z_{2}\right)^{k} Y\left(u, z_{0}+z_{2}\right) Y\left(v, z_{2}\right) w=\lambda\left(z_{2}+z_{0}\right)^{k} Y\left(Y\left(u, z_{0}\right) v, z_{2}\right) w .
$$

We shall show that the associativity above leads to the locality. The idea of the following argument comes from $[\mathrm{R}]$. Let $N \in \mathbb{Z}$ such that $z^{N} Y(u, z) v \in V^{0} \llbracket z \rrbracket$. Take sufficiently large $s, t \in \mathbb{Z}$ such that $z^{s} Y(v, z) w \in V^{0} \llbracket z \rrbracket$ and (2.3) holds for $(u, v, w)$ and $(v, u, w)$ with $k=t, s$. Then

$$
\begin{aligned}
& z_{1}^{t} z_{2}^{s}\left(z_{1}-z_{2}\right)^{N} Y\left(u, z_{1}\right) Y\left(v, z_{2}\right) w \\
& =e^{-z_{2} \partial_{z_{1}}}\left(\left(z_{1}+z_{2}\right)^{t} z_{2}^{s} z_{1}^{N} Y\left(u, z_{1}+z_{2}\right) Y\left(v, z_{2}\right) w\right) \\
& =\lambda e^{-z_{2} \partial_{z_{1}}}\left(\left(z_{2}+z_{1}\right)^{t} z_{2}^{s} z_{1}^{N} Y\left(Y\left(u, z_{1}\right) v, z_{2}\right) w\right) \\
& =\lambda e^{-z_{2} \partial_{z_{1}}}\left(\left(z_{2}+z_{1}\right)^{t} z_{2}^{s} z_{1}^{N} Y\left(e^{z_{1} L(-1)} Y\left(v,-z_{1}\right) u, z_{2}\right) w\right) \\
& =\lambda e^{-z_{2} \partial_{z_{1}}} e^{z_{1} \partial_{z_{2}}}\left(z_{2}^{t}\left(z_{2}-z_{1}\right)^{s} z_{1}^{N} Y\left(Y\left(v,-z_{1}\right) u, z_{2}\right) w\right) .
\end{aligned}
$$

Define $p\left(z_{1}, z_{2}\right):=z_{2}^{t}\left(z_{2}-z_{1}\right)^{s} z_{1}^{N} Y\left(Y\left(v,-z_{1}\right) u, z_{2}\right) w$. The equations above show that $p(z, w) \in V^{1} \llbracket z_{1}, z_{2} \rrbracket$. On the other hand,

$$
\begin{aligned}
& z_{1}^{t} z_{2}^{s}\left(-z_{2}+z_{1}\right)^{N} Y\left(v, z_{2}\right) Y\left(u, z_{1}\right) w \\
& =e^{-z_{1} \partial_{z_{2}}}\left(z_{1}^{t}\left(z_{2}+z_{1}\right)^{s}\left(-z_{2}\right)^{N} Y\left(v, z_{2}+z_{1}\right) Y\left(u, z_{1}\right) w\right) \\
& =\lambda e^{-z_{1} \partial_{z_{2}}}\left(z_{1}^{t}\left(z_{1}+z_{2}\right)^{s}\left(-z_{2}\right)^{N} Y\left(Y\left(v, z_{2}\right) u, z_{1}\right) w\right) \\
& =\lambda e^{-z_{1} \partial_{z_{2}}} p\left(-z_{2}, z_{1}\right) .
\end{aligned}
$$

Thus the locality follows from

$$
e^{-w \partial_{z}} e^{z \partial_{w}} p(z, w)=e^{-w \partial_{z}} p(z, w+z)=e^{-w \partial_{z}} p(z, z+w)=p(z-w, z)
$$

and $e^{-z \partial_{w}} p(-w, z)=p(-w+z, z)=p(z-w, z)$.

The uniqueness has already been shown in DM2] in a general fashion.

Later, we shall consider a construction of framed VOAs. The following extension property will be used frequently. 
Theorem 2.7. (Extension property [Y2, Theorem 4.6.1]) Let $V^{(0,0)}$ be a simple rational $C_{2}$-cofinite VOA of CFT-type, and let $D_{1}, D_{2}$ be finite abelian groups. Assume that we have a set of inequivalent irreducible simple current $V^{(0,0)}$-modules $\left\{V^{(\alpha, \beta)} \mid(\alpha, \beta) \in\right.$ $\left.D_{1} \oplus D_{2}\right\}$ with $D_{1} \oplus D_{2}$-graded fusion rules $V^{\left(\alpha_{1}, \beta_{1}\right)} \bigotimes_{V^{(0,0)}} V^{\left(\alpha_{2}, \beta_{2}\right)}=V^{\left(\alpha_{1}+\alpha_{2}, \beta_{1}+\beta_{2}\right)}$ for any $\left(\alpha_{1}, \beta_{1}\right),\left(\alpha_{2}, \beta_{2}\right) \in D_{1} \oplus D_{2}$. Further assume that all $V^{(\alpha, \beta)},(\alpha, \beta) \in D_{1} \oplus D_{2}$, have integral top weights and we have $D_{1}$ - and $D_{2}$-graded simple current extensions $V_{D_{1}}=\oplus_{\alpha \in D_{1}} V^{(\alpha, 0)}$

and $V_{D_{2}}=\oplus_{\beta \in D_{2}} V^{(0, \beta)}$. Then $V_{D_{1} \oplus D_{2}}:=\oplus_{(\alpha, \beta) \in D_{1} \oplus D_{2}} V^{(\alpha, \beta)}$ possesses a unique structure of a simple vertex operator algebra as a $\left(D_{1} \oplus D_{2}\right)$-graded simple current extension of $V^{(0,0)}$.

\section{Ising frame and framed VOA}

We shall review the notion of an Ising frame and a framed vertex operator algebra.

\subsection{Miyamoto involutions}

We begin by the definition of an Ising vector.

Definition 3.1. A Virasoro vector $e$ is called an Ising vector if $\operatorname{Vir}(e) \simeq L(1 / 2,0)$. Two Virasoro vectors $u, v \in V$ are called orthogonal if $\left[Y\left(u, z_{1}\right), Y\left(v, z_{2}\right)\right]=0$. A decomposition $\omega=e^{1}+\cdots+e^{n}$ of the conformal vector $\omega$ of $V$ is called orthogonal if $e^{i}$ are mutually orthogonal Virasoro vectors.

Let $e \in V$ be an Ising vector. By definition, $\operatorname{Vir}(e) \simeq L(1 / 2,0)$. It is well-known that $L(1 / 2,0)$ is rational, $C_{2}$-cofinite and has three irreducible modules $L(1 / 2,0), L(1 / 2,1 / 2)$ and $L(1 / 2,1 / 16)$. The fusion rules of $L(1 / 2,0)$-modules are computed in [DMZ]:

$$
\begin{aligned}
& L(1 / 2,1 / 2) \otimes L(1 / 2,1 / 2)=L(1 / 2,0), \quad L(1 / 2,1 / 2) \otimes L(1 / 2,1 / 16)=L(1 / 2,1 / 16), \\
& L(1 / 2,1 / 16) \otimes L(1 / 2,1 / 16)=L(1 / 2,0) \oplus L(1 / 2,1 / 2) .
\end{aligned}
$$

By (3.1), one can define some involutions in the following way. Let $V_{e}(h)$ be the sum of all irreducible $\operatorname{Vir}(e)$-submodules of $V$ isomorphic to $L(1 / 2, h)$ for $h=0,1 / 2,1 / 16$. Then one has the isotypical decomposition

$$
V=V_{e}(0) \oplus V_{e}(1 / 2) \oplus V_{e}(1 / 16)
$$

Define a linear automorphism $\tau_{e}$ on $V$ by

$$
\tau_{e}=\left\{\begin{array}{rll}
1 & \text { on } \quad V_{e}(0) \oplus V_{e}(1 / 2) \\
-1 & \text { on } \quad V_{e}(1 / 16)
\end{array}\right.
$$


Then by the fusion rules in (3.1), $\tau_{e}$ defines an automorphism on the VOA $V$ (cf. [M1]). On the fixed point subalgebra $V^{\left\langle\tau_{e}\right\rangle}=V_{e}(0) \oplus V_{e}(1 / 2)$, one can define another linear automorphism $\sigma_{e}$ by

$$
\sigma_{e}=\left\{\begin{array}{rll}
1 & \text { on } & V_{e}(0) \\
-1 & \text { on } & V_{e}(1 / 2)
\end{array}\right.
$$

Then $\sigma_{e}$ also defines an automorphism on $V^{\left\langle\tau_{e}\right\rangle}$ (cf. [M1]). The automorphisms $\tau_{e} \in$ $\operatorname{Aut}(V)$ and $\sigma_{e} \in \operatorname{Aut}\left(V^{\left\langle\tau_{e}\right\rangle}\right)$ are often called Miyamoto involutions.

\subsection{Framed VOAs and their structure codes}

Let us define the notion of a framed VOA.

Definition 3.2. ([DGH, $\mathrm{M} 3])$ A simple vertex operator algebra $(V, \omega)$ is called framed if there exists a set $\left\{e^{1}, \ldots, e^{n}\right\}$ of Ising vectors of $V$ such that $\omega=e^{1}+\cdots+e^{n}$ is an orthogonal decomposition. The full sub VOA $F$ generated by $e^{1}, \ldots, e^{n}$ is called an Ising frame or simply a frame of $V$. By abuse of notation, we sometimes call the set of Ising vectors $\left\{e^{1}, \ldots, e^{n}\right\}$ a frame, also.

Let $(V, \omega)$ be a framed VOA with an Ising frame $F$. Then

$$
F \simeq \operatorname{Vir}\left(e^{1}\right) \otimes \cdots \otimes \operatorname{Vir}\left(e^{i}\right) \simeq L(1 / 2,0)^{\otimes n}
$$

and $V$ is a direct sum of irreducible $F$-submodules $\otimes_{i=1}^{n} L\left(1 / 2, h_{i}\right)$ with $h_{i} \in\{0,1 / 2,1 / 16\}$. For each irreducible $F$-module $W=\otimes_{i=1}^{n} L\left(1 / 2, h_{i}\right)$, we define its binary 1/16-word (or $\tau$-word $) \tau(W)=\left(\alpha_{1}, \cdots, \alpha_{n}\right) \in \mathbb{Z}_{2}^{n}$ by $\alpha_{i}=1$ if and only if $h_{i}=1 / 16$. For $\alpha \in \mathbb{Z}_{2}^{n}$, denote by $V^{\alpha}$ the sum of all irreducible $F$-submodules of $V$ whose $1 / 16$-words are equal to $\alpha$. Define a linear code $D \subset \mathbb{Z}_{2}^{n}$ by $D=\left\{\alpha \in \mathbb{Z}_{2}^{n} \mid V^{\alpha} \neq 0\right\}$. Then we have the $1 / 16$-word decomposition $V=\oplus_{\alpha \in D} V^{\alpha}$. By the fusion rules of $L(1 / 2,0)$-modules, it is easy to see that $V^{\alpha} \cdot V^{\beta} \subset V^{\alpha+\beta}$. Hence, the dual group $D^{*}$ of $D$ acts on $V$. In fact, the action of $D^{*}$ coincides with the action of the elementary abelian 2-group generated by Miyamoto involutions $\left\{\tau_{e^{i}} \mid 1 \leq i \leq n\right\}$. Therefore, all $V^{\alpha}, \alpha \in D$, are irreducible $V^{0}$-modules by DM1. Since there is no $L(1 / 2,1 / 16)$-component in $V^{0}$, the fixed point subalgebra $V^{D^{*}}=V^{0}$ has the following shape:

$$
V^{0}=\bigoplus_{h_{i} \in\{0,1 / 2\}} m_{h_{1}, \ldots, h_{n}} L\left(1 / 2, h_{1}\right) \otimes \cdots \otimes L\left(1 / 2, h_{n}\right)
$$

where $m_{h_{1}, \ldots, h_{n}} \in \mathbb{N}$ denotes the multiplicity. On $V^{0}$ we can define Miyamoto involutions $\sigma_{e^{i}}$ for $i=1, \ldots, n$. Denote by $Q$ the elementary abelian 2-subgroup of $\operatorname{Aut}\left(V^{0}\right)$ generated by $\left\{\sigma_{e^{i}} \mid 1 \leq i \leq n\right\}$. Then the fixed point subalgebra $\left(V^{0}\right)^{Q}=F$ and each $m_{h_{1}, \cdots, h_{n}} L\left(1 / 2, h_{1}\right) \otimes \cdots \otimes L\left(1 / 2, h_{n}\right)$ is an irreducible $F$-submodule again by [DM1]. Thus 
$m_{h_{1}, \cdots, h_{n}} \in\{0,1\}$ and we obtain an even linear code $C:=\left\{\left(2 h_{1}, \cdots, 2 h_{n}\right) \in \mathbb{Z}_{2}^{n} \mid h_{i} \in\right.$ $\left.\{0,1 / 2\}, m_{h_{1}, \cdots, h_{n}} \neq 0\right\}$, namely,

$$
V^{0}=\bigoplus_{\alpha=\left(\alpha_{1}, \cdots, \alpha_{n}\right) \in C} L\left(1 / 2, \alpha_{1} / 2\right) \otimes \cdots \otimes L\left(1 / 2, \alpha_{n} / 2\right) .
$$

Since $L(1 / 2,0)$ and $L(1 / 2,1 / 2)$ are simple current $L(1 / 2,0)$-modules, $V^{0}$ is a $C$-graded simple current extension of $F$. By Proposition 2.3, the simple VOA structure on $V^{0}$ is unique. The simple VOA $V^{0}$ of the form (3.2) is called the code VOA associated to $C$ and denoted by $V_{C}$. It is clear that $V_{C}$ is simple, rational, $C_{2}$-cofinite and of CFT-type. Since $L(1)\left(V_{C}\right)_{1}=0$ by its $F$-module structure, $V_{C}$ has a non-zero invariant form and thus is self-dual as a $V_{C}$-module by [Li1]. Similarly, we also have $L(1) V_{1}=0$ and $V$ is self-dual as a $V$-module.

Summarizing, there exists a pair $(C, D)$ of even linear codes such that $V$ is an $D$-graded extension of a code VOA $V_{C}$ associated to $C$. We call the pair $(C, D)$ the structure codes of a framed VOA $V$ associated with the frame $F$. Since the powers of $z$ in an $L(1 / 2,0)$-intertwining operator of type $L(1 / 2,1 / 2) \times L(1 / 2,1 / 2) \rightarrow L(1 / 2,1 / 16)$ are half-integral, the structure codes $(C, D)$ satisfy $C \subset D^{\perp}$.

Notation Let $V$ be a framed VOA with the structure codes $(C, D)$, where $C, D \subset \mathbb{Z}_{2}^{n}$. For a binary codeword $\beta \in \mathbb{Z}_{2}^{n}$, we define:

$$
\sigma_{\beta}:=\prod_{i \in \operatorname{supp}(\beta)} \sigma_{e^{i}} \in \operatorname{Aut}\left(V^{0}\right) \quad \text { and } \quad \tau_{\beta}:=\prod_{i \in \operatorname{supp}(\beta)} \tau_{e^{i}} \in \operatorname{Aut}(V) .
$$

Namely, by associating Miyamoto involutions to a codeword of $\mathbb{Z}_{2}^{n}, \sigma: \mathbb{Z}_{2}^{n} \rightarrow \operatorname{Aut}\left(V^{0}\right)$ and $\tau: \mathbb{Z}_{2}^{n} \rightarrow \operatorname{Aut}(V)$ define group homomorphisms. It is also clear that $\operatorname{ker} \sigma=C^{\perp}$ and $\operatorname{ker} \tau=D^{\perp}$.

\section{Representation of code VOAs}

Since every framed VOA is an extension of its code sub VOA, it is quite natural to study a framed VOA as a module over its code sub VOA. Let us first review a structure theory for the irreducible modules over a code VOA.

\subsection{Central extension of codes}

Let $\nu^{1}=(10 \ldots 0), \nu^{2}=(010 \ldots 0), \ldots, \nu^{n}=(0 \ldots 01) \in \mathbb{Z}_{2}^{n}$. Define $\varepsilon: \mathbb{Z}_{2}^{n} \times \mathbb{Z}_{2}^{n} \rightarrow \mathbb{C}^{*}$ by

$$
\varepsilon\left(\nu^{i}, \nu^{j}\right):=-1 \quad \text { if } \quad i>j \quad \text { and } 1 \text { otherwise, }
$$

and extend to $\mathbb{Z}_{2}^{n}$ linearly. Then $\varepsilon$ defines a 2-cocycle in $Z^{2}\left(\mathbb{Z}_{2}^{n}, \mathbb{C}^{*}\right)$. By definition,

$$
\varepsilon(\alpha, \beta) \varepsilon(\beta, \alpha)=(-1)^{\langle\alpha, \beta\rangle+\operatorname{wt}(\alpha) \operatorname{wt}(\beta)} \text { and } \varepsilon(\alpha, \alpha)=(-1)^{\operatorname{wt}(\alpha)(\operatorname{wt}(\alpha)-1) / 2}
$$


for all $\alpha, \beta \in \mathbb{Z}_{2}^{n}$. In particular, $\varepsilon(\alpha, \alpha)=(-1)^{\operatorname{wt}(\alpha) / 2}$ and $\varepsilon(\alpha, \beta) \varepsilon(\beta, \alpha)=(-1)^{\langle\alpha, \beta\rangle}$ if $\alpha, \beta \in \mathbb{Z}_{2}^{n}$ are even.

Let $G$ be the central extension of $\mathbb{Z}_{2}^{n}$ by $\mathbb{C}^{*}$ with associated 2-cocycle $\varepsilon$. Recall that $G=\mathbb{Z}_{2}^{n} \times \mathbb{C}^{*}$ as a set, but the group operation is given by

$$
(\alpha, u)(\beta, v)=(\alpha \beta, \varepsilon(\alpha, \beta) u v)
$$

for all $\alpha, \beta \in \mathbb{Z}_{2}^{n}$ and $u, v \in \mathbb{C}^{*}$. Let $C$ be a binary even linear code of $\mathbb{Z}_{2}^{n}$. Since $\varepsilon$ takes values in $\{ \pm 1\}$, we can take a subgroup $\tilde{C}=\{(\alpha, u) \in G \mid \alpha \in C, u \in\{ \pm 1\}\}$ of $G$ so that $\tilde{C}$ forms a central extension of $C$ by $\{ \pm 1\}$ :

$$
1 \longrightarrow\{ \pm 1\} \longrightarrow \tilde{C} \stackrel{\pi}{\longrightarrow} C \longrightarrow 1
$$

We shall note that the radical of the standard bilinear form $\langle\cdot, \cdot\rangle$ on $C$ is given by $R=$ $C \cap C^{\perp}$ and thus by (4.2), the preimage $\tilde{R}=\pi^{-1}\left(C \cap C^{\perp}\right)$ is the center of $\tilde{C}$. Take a subgroup $D$ of $C$ such that $C=R \oplus D$. Then the form $\langle\cdot, \cdot\rangle$ is non-degenerate on $D$. It follows from (4.2) that the preimage $\tilde{D}:=\pi^{-1}(D)$ is an extra-special 2-subgroup of $\tilde{C}$. The central extension $\tilde{C}$ is then isomorphid 3 to the central product of $\tilde{D}$ and $\tilde{R}$ over $\{ \pm 1\} \subset \mathbb{C}^{*}$ which we shall denote by $\tilde{D} *_{\{ \pm 1\}} \tilde{R}$.

We identify the multiplicative group $\mathbb{C}^{*}$ with the central subgroup $\left(0, \mathbb{C}^{*}\right)=\{(0, u) \in$ $\left.G \mid u \in \mathbb{C}^{*}\right\}$ of $G$, and let $\mathbb{C}^{*} \tilde{C}=\left\{(\alpha, u) \in G \mid \alpha \in C, u \in \mathbb{C}^{*}\right\}$ be the subgroup of $G$ generated by $\mathbb{C}^{*}=\left(0, \mathbb{C}^{*}\right)$ and $\tilde{C}$. Then we have the exact sequence:

$$
1 \longrightarrow \mathbb{C}^{*} \longrightarrow \mathbb{C}^{*} \tilde{C} \stackrel{\pi_{\mathbb{C}^{*}}}{\longrightarrow} C \longrightarrow 1
$$

Since $\mathbb{C}^{*}$ is injective in the category of abelian groups, the preimage of $C \cap C^{\perp}$ in $\mathbb{C}^{*} \tilde{C}$ splits and one has an isomorphism

$$
\mathbb{C}^{*} \tilde{C} \simeq\left(C \cap C^{\perp}\right) \times\left(\mathbb{C}^{*} *_{\{ \pm 1\}} \tilde{D}\right)
$$

Now let $\psi: C \rightarrow \operatorname{End}(V)$ be a $\varepsilon$-projective representation of $C$ on $V$, that is, $\psi(\alpha) \psi(\beta)=\varepsilon(\alpha, \beta) \psi(\alpha+\beta)$ for $\alpha, \beta \in C$. Then one defines a linear representation $\tilde{\psi}$ of $\mathbb{C}^{*} \tilde{C}$ via $\tilde{\psi}(\alpha, u):=u \psi(\alpha) \in \operatorname{End}(V)$ for $\alpha \in C$ and $u \in \mathbb{C}^{*}$. Since $\mathbb{C}^{*} \tilde{C}$ is isomorphic to a direct product of $R=C \cap C^{\perp}$ and $\mathbb{C}^{*} *_{\{ \pm 1\}} \tilde{D}, \tilde{\psi}$ is a tensor product of a linear character of $R$ and an irreducible non-linear character of $\tilde{D}$ if $\tilde{\psi}$ is irreducible. Since $\tilde{D}$ is an

\footnotetext{
${ }^{3}$ Note that the isomorphism type of $\tilde{D}$ is determined by the dimension of maximal isotropic subspaces of $D$ with respect to the quadratic form $q(\alpha)=\varepsilon(\alpha, \alpha)$ (cf. [Go] and [FLM], Section 5.3]), which depends on the choice of the complement $D$ if $R$ is not doubly even. For example, we can take $C=\operatorname{Span}_{\mathbb{Z}_{2}}\{(11000),(00110),(00101)\}$. Then the radical $R=\{(00000),(11000)\}$. Set $D=\{(00000),(00110),(00101),(00011)\}$ and $D^{\prime}=\{(00000),(11110),(00101),(11011)\}$. Then both $D$ and $D^{\prime}$ are complement of $R$ in $C$ but $\tilde{D} \nsucc \tilde{D}^{\prime}$, for $\tilde{D}$ is a quaternion group whereas $\tilde{D}^{\prime}$ is a dihedral group of order 8 . Nevertheless, the central product $\tilde{D} *_{\{ \pm 1\}} \tilde{R}$ is still uniquely determined by $C$ up to isomorphisms.
} 
extra-special 2 group, $D$ has only one non-linear irreducible character up to isomorphisms (cf. [Go] and [FLM, Theorem 5.5.1]). Therefore, the number of inequivalent irreducible $\varepsilon$-projective representation of $C$ is equal to the order of $R=C \cap C^{\perp}$.

Let us review the structure of the irreducible non-linear character of $\tilde{D}$ in more detail. Let $H$ be a maximal self-orthogonal subcode of $D$. Then by (4.2) the preimage $\pi_{\mathbb{C}^{*}}^{-1}(H)$ of $H$ in $\mathbb{C}^{*} \tilde{C}$ splits. Hence, there exists a map $\iota: H \rightarrow \mathbb{C}^{*}$ such that $\varepsilon(\alpha, \beta)=(\partial \iota)(\alpha, \beta)=\iota(\alpha) \iota(\beta) / \iota(\alpha+\beta)$ for all $\alpha, \beta \in H$. Since $\varepsilon(\alpha, \beta) \in\{ \pm 1\}$, one also has $\varepsilon(\alpha, \beta)=\varepsilon(\alpha, \beta)^{-1}=\iota(\alpha+\beta) / \iota(\alpha) \iota(\beta)$. Then the section map $H \ni \alpha \mapsto(\alpha, \iota(\alpha)) \in$ $\pi_{\mathbb{C}^{*}}^{-1}(H)$ is a group homomorphism. Let $\chi$ be a linear character of $H$ and define a linear character $\tilde{\chi}$ of $\pi_{\mathbb{C}^{*}}^{-1}(H)$ by $\tilde{\chi}(\alpha, \iota(\alpha) u)=u \chi(\alpha)$ for $\alpha \in H$ and $u \in \mathbb{C}^{*}$. Since the preimage $\tilde{H}:=\pi^{-1}(H)$ is a subgroup of $\pi_{\mathbb{C}^{*}}^{-1}(H)$, we may view $\tilde{\chi}$ as a linear character of $\tilde{H}$. Then the irreducible non-linear character of $\tilde{D}$ is realized by the induced module $\operatorname{Ind}_{\tilde{H}}^{\tilde{D}} \tilde{\chi}(\operatorname{cf}$. Theorem 5.5.1 of [FLM] ). Summarizing, we have:

Proposition 4.1. (Theorem 5.5.1 of $[F L M])$ Let $\psi$ be an irreducible $\varepsilon$-projective representation of $C$. Then the associated linear representation $\tilde{\psi}$ of $\mathbb{C}^{*} \tilde{C}$ is of the form $\lambda \otimes_{\mathbb{C}} \operatorname{Ind}_{\tilde{H}}^{\tilde{D}} \tilde{\chi}$, where $\lambda$ is a linear character of $C \cap C^{\perp}, \tilde{H}$ is the preimage of a maximal self-orthogonal subcode $H$ of $D$ in $\tilde{C}$, and $\tilde{\chi}$ is a linear character of $\tilde{H}$ such that $\tilde{\chi}(0,-1)=-1$. In particular, $\tilde{\psi}$ is induced from a linear character of a maximal abelian subgroup of $\tilde{C}$.

\subsection{Structure of modules}

Let $C$ be an even linear code of $\mathbb{Z}_{2}^{n}$. For a codeword $\alpha=\left(\alpha_{1}, \ldots, \alpha_{n}\right) \in C$, we set

$$
V(\alpha):=L\left(1 / 2, \alpha_{1} / 2\right) \otimes \cdots \otimes L\left(1 / 2, \alpha_{n} / 2\right)
$$

Let $V_{C}=\oplus_{\alpha \in C} V(\alpha)$ be the code VOA associated to $C$. Since $V(0)=L(1 / 2,0)^{\otimes n}$ is a rational full sub VOA of $V_{C}$, every $V_{C}$-module is completely reducible as a $V(0)$-module. We shall review the structure theory of irreducible $V_{C}$-modules from [M2, L3, Y1, Y2].

Let $M$ be an irreducible $V_{C}$-module. Take an irreducible $V(0)$-submodule $W$ of $M$, which is possible as $V(0)$ is rational. Let $\tau(W) \in \mathbb{Z}_{2}^{n}$ be the binary $1 / 16$-word of $W$ as defined in (1.1) (see also Section [3.2). Then it follows from the fusion rules of $L(1 / 2,0)$ modules that $\tau(W) \in C^{\perp}$ and $\tau(W)=\tau\left(W^{\prime}\right)$ for any irreducible $V(0)$-submodule $W^{\prime}$ of $M$. Set $C_{W}:=\left\{\alpha \in C \mid V(\alpha) \bigotimes_{V(0)} W \simeq W\right\}$. Then $C_{W}=\{\alpha \in C \mid \operatorname{supp}(\alpha) \subset$ $\operatorname{supp}(\tau(W))\}$ and $C_{W^{\prime}}=C_{W}$ for any irreducible $V(0)$-submodule $W^{\prime}$ of $M$. Let $\left\{\alpha_{i} \mid\right.$ $1 \leq i \leq r\}$ be the coset representatives for $C_{W}$ in $C$. By the definition of $C_{W}$, it follows $V\left(\alpha_{i}\right) \bigotimes_{V(0)} W \not V\left(\alpha_{j}\right) \bigotimes_{V(0)} W$ if $i \neq j$, because if $V(\beta) \bigotimes_{V(0)} W=V(\gamma) \bigotimes_{V(0)} W$ in the fusion algebra then $W=V(\beta) \bigotimes_{V(0)} V(\gamma) \bigotimes_{V(0)} W=V(\beta+\gamma) \bigotimes_{V(0)} W$ for $\beta, \gamma \in C$. Note that the fusion algebra associated to $V(0)$ is associative and $V(\beta) \bigotimes_{V(0)} V(\gamma)=$ $V(\beta+\gamma)$. For simplicity, we set $W^{i}:=V\left(\alpha_{i}\right) \bigotimes_{V(0)} W$. Then we have the following 
isotypical decomposition:

$$
M=\bigoplus_{i=1}^{r} W^{i} \otimes \operatorname{Hom}_{V(0)}\left(W^{i}, M\right) .
$$

In the decomposition above, each homogeneous component

$$
W^{i} \otimes \operatorname{Hom}_{V(0)}\left(W^{i}, M\right)
$$

of $M$ forms an irreducible $V_{C_{W}}$-submodule, where $V_{C_{W}}$ is the code VOA associated to $C_{W}$. Let $U:=\operatorname{Hom}_{V(0)}(W, M)$. It is shown in [M2, L3, Y2] that $U$ is an irreducible $\varepsilon$ projective representation of $C_{W}$ so that $U$ is also an irreducible $\mathbb{C}^{*} \tilde{C}_{W^{-}}$-module. Moreover, the $V_{C}$-module structure on $M$ is uniquely determined by the $\mathbb{C}^{*} \tilde{C}_{W}$-module structure on $U$.

Theorem 4.2. ([M2, L3, [Y2] $]$ ) Let $C$ be an even linear code and $V_{C}=\oplus_{\alpha \in C} V(\alpha)$ the associated code VOA. Let $W$ be an irreducible $V(0)$-module such that $\tau(W) \in C^{\perp}$. Then there is a one to one correspondence between the isomorphism classes of irreducible $\varepsilon$ projective representations of $C_{W}$ and the isomorphism classes of irreducible $V_{C}$-modules containing $W$ as a $V^{0}$-submodule.

In the following, we shall give an explicit construction of irreducible $V_{C}$-modules from irreducible $\varepsilon$-projective $C_{W}$-modules.

An explicit construction Let $W$ be an irreducible $V(0)$-module such that the $1 / 16$ word $\tau(W) \in C^{\perp}$. Let $H$ be a maximal self-orthogonal subcode of $C_{W}=\{\alpha \in C \mid$ $\operatorname{supp}(\alpha) \subset \operatorname{supp}(\tau(W))\}$. Since the preimage $\pi_{\mathbb{C}^{*}}^{-1}(H)$ of $H$ in (4.4) splits, there is a map $\iota: H \rightarrow \mathbb{C}^{*}$ such that $(\alpha, \iota(\alpha))(\beta, \iota(\beta))=(\alpha+\beta, \iota(\alpha+\beta))$ for all $\alpha, \beta \in H$. Let $\chi$ be a linear character of $H$. Then we can define a linear character $\tilde{\chi}_{\iota}$ of $\pi_{\mathbb{C}^{*}}^{-1}(H)$ by

$$
\tilde{\chi}_{\iota}(\alpha, \iota(\alpha) u)=u \chi(\alpha) \quad \text { for } \alpha \in H, u \in \mathbb{C}^{*} .
$$

In this case, $\tilde{\chi}_{\iota}$ is also a linear character on the preimage $\tilde{H}=\pi^{-1}(H)$ of $H$ in (4.3). Let $\mathbb{C}^{\varepsilon}[C]$ be the twisted group algebra associated to the 2-cocycle $\varepsilon \in Z^{2}\left(C, \mathbb{C}^{*}\right)$ defined in (4.1). That means $\mathbb{C}^{\varepsilon}[C]=\operatorname{Span}_{\mathbb{C}}\left\{e^{\alpha} \mid \alpha \in C\right\}$ as a linear space and $e^{\alpha} e^{\beta}=\varepsilon(\alpha, \beta) e^{\alpha+\beta}$. By (4.2), we have

$$
e^{\alpha} e^{\beta}=(-1)^{\langle\alpha, \beta\rangle} e^{\beta} e^{\alpha} .
$$

It is clear that $\mathbb{C}^{\varepsilon}\left[C_{W}\right]=\oplus_{\alpha \in C_{W}} \mathbb{C} e^{\alpha}$ and $\mathbb{C}^{\varepsilon}[H]=\oplus_{\beta \in H} \mathbb{C} e^{\beta}$ are subalgebras of $\mathbb{C}^{\varepsilon}[C]$. Moreover, $\mathbb{C}^{\varepsilon}[H] \simeq \mathbb{C}[H]$ as $\mathbb{C}$-algebras. Let $\left\{\alpha_{1}, \ldots, \alpha_{r}\right\}$ be a set of coset representatives for $C_{W}$ in $C$ and let $\left\{\beta_{1}, \ldots, \beta_{s}\right\}$ be a set of coset representatives for $H$ in $C_{W}$. Consider an induced module $\operatorname{Ind}_{\tilde{H}}^{\tilde{C}} \tilde{\chi}_{\iota}$. As a linear space, it is defined by

$$
\operatorname{Ind}_{\tilde{H}}^{\tilde{C}} \tilde{\chi}_{\iota}=\bigoplus_{i=1}^{r} \bigoplus_{j=1}^{s} \mathbb{C} e^{\alpha_{i}+\beta_{j}} \underset{\mathbb{C}^{\varepsilon}[\tilde{H}]}{\otimes} v_{\tilde{\chi}_{\iota}},
$$


where $\mathbb{C} v_{\tilde{\chi}_{\iota}}$ is a $\mathbb{C}^{\varepsilon}[H]$-module affording the character $\tilde{\chi}_{\iota}$, that is, $\iota(\alpha) e^{\alpha} \cdot v_{\tilde{\chi}_{\iota}}=\chi(\alpha) v_{\tilde{\chi}_{\iota}}$ for all $\alpha \in H$. Note also that the components

$$
U^{i}:=\bigoplus_{j=1}^{s} \mathbb{C} e^{\alpha_{i}+\beta_{j}} \underset{\mathbb{C}^{\varepsilon}[\tilde{H}]}{\otimes} v_{\tilde{\chi}_{\iota}}, \quad 1 \leq i \leq r,
$$

are irreducible $\mathbb{C}^{\varepsilon}\left[C_{W}\right]$-modules. Set $W^{i}:=V\left(\alpha_{i}\right) \bigotimes_{V(0)} W$ for $1 \leq i \leq r$. Let $I^{\alpha, i}(\cdot, z)$ be a $V(0)$-intertwining operator of type $V(\alpha) \times W^{i} \rightarrow V(\alpha) \bigotimes_{V(0)} W^{i}$. Since all $V(\alpha)$, $\alpha \in C$, are simple current $V(0)$-modules, $I^{\alpha, i}(\cdot, z)$ are unique up to scalars. It is possible to choose these intertwining operators such that

$$
\begin{aligned}
& \left(z_{0}+z_{2}\right)^{m} I^{\alpha, j^{\prime}}\left(x^{\alpha}, z_{0}+z_{2}\right) I^{\beta, j}\left(x^{\beta}, z_{2}\right) w^{j} \\
& =\varepsilon(\alpha, \beta)\left(z_{2}+z_{0}\right)^{m} I^{\alpha+\beta, j}\left(Y_{V_{C}}\left(x^{\alpha}, z_{0}\right) x^{\beta}, z_{2}\right) w^{j}
\end{aligned}
$$

for $x^{\alpha} \in V^{\alpha}, x^{\beta} \in V^{\beta}, w^{j} \in W^{j}, \alpha_{j^{\prime}}+C_{W}=\beta+\alpha_{j}+C_{W}$ and $m \gg 0$ (cf. [M2, [Y2]). We can also choose $I^{0, i}(\cdot, z)$ so that $I^{0, i}(\mathbb{1}, z)=\operatorname{id}_{W^{i}}$. Now put

$$
M=\operatorname{Ind}_{V_{H}}^{V_{C}}\left(W, \tilde{\chi}_{\iota}\right):=\bigoplus_{i=1}^{r} W^{i} \underset{\mathbb{C}}{\otimes} U^{i}
$$

and define a vertex operator $Y(\cdot, z): V_{C} \times M \rightarrow M((z))$ by

$$
Y\left(x^{\alpha}, z\right) w^{i} \underset{\mathbb{C}}{\otimes} u^{i}:=I^{\alpha, i}\left(x^{\alpha}, z\right) w^{i} \underset{\mathbb{C}}{\otimes}\left(e^{\alpha} \cdot u^{i}\right)
$$

for $x^{\alpha} \in V^{\alpha}, w^{i} \in W^{i}$ and $u^{i} \in U^{i}$.

Theorem 4.3. ([M2, L3, [Y1] ) The induced module $\operatorname{Ind}_{V^{0}}^{V_{C}}\left(W, \tilde{\chi}_{\iota}\right)$ equipped with the vertex operator defined above is an irreducible $V_{C}$-module. Moreover, every irreducible $V_{C}$-module is isomorphic to an induced module.

Remark 4.4. Even if $\tau(W) \notin C^{\perp}$, one can still define an irreducible $\mathbb{Z}_{2}$-twisted $V_{C^{-}}$ module structure on $\operatorname{Ind}_{V_{H}}^{V_{C}}(W, \tilde{\chi})(c f .[L 1,[Y 1])$.

Parameterization by a pair of binary codewords The irreducible $V_{C}$-modules can also be parameterized by a pair of binary codewords. For given $\beta \in C^{\perp}$ and $\gamma \in \mathbb{Z}_{2}^{n}$, we define a weight vector $h_{\beta, \gamma}=\left(h_{\beta, \gamma}^{1}, \ldots, h_{\beta, \gamma}^{n}\right), h_{\beta, \gamma}^{i} \in\{0,1 / 2,1 / 16\}$ by

$$
h_{\beta, \gamma}^{i}:= \begin{cases}\frac{1}{16} & \text { if } \beta_{i}=1 \\ \frac{\gamma_{i}}{2} & \text { if } \beta_{i}=0 .\end{cases}
$$

Let

$$
L\left(h_{\beta, \gamma}\right):=L\left(1 / 2, h_{\beta, \gamma}^{1}\right) \otimes \cdots \otimes L\left(1 / 2, h_{\beta, \gamma}^{n}\right)
$$


be the irreducible $L(1 / 2,0)^{\otimes n}$-module with the weight $h_{\beta, \gamma}$. Set $C_{\beta}:=\{\alpha \in C \mid \operatorname{supp}(\alpha) \subset$ $\operatorname{supp}(\beta)\}$ and let $R^{\beta}=C_{\beta} \cap\left(C_{\beta}\right)^{\perp}$ be the radical of $C_{\beta}$. Fix a map $\iota: R^{\beta} \rightarrow \mathbb{C}^{*}$ such that the section map $R^{\beta} \ni \alpha \mapsto(\alpha, \iota(\alpha)) \in \pi_{\mathbb{C}^{*}}^{-1}\left(R^{\beta}\right)$ is a group homomorphism. Take a maximal self-orthogonal subcode $H$ of $C_{\beta}$. Then $R^{\beta} \subset H$ and we can extend $\iota$ to $H$ such that the section map $H \ni \alpha \mapsto(\alpha, \iota(\alpha)) \in \pi_{\mathbb{C}^{*}}^{-1}(H)$ is a group homomorphism. For, there exists a map $\jmath: H \rightarrow \mathbb{C}^{*}$ such that $H \ni \alpha \mapsto(\alpha, \jmath(\alpha)) \in \pi_{\mathbb{C}^{*}}^{-1}(H)$ is a group homomorphism. Then $\mu=\iota / \jmath$ restricted on $R^{\beta}$ is a character since $\partial \mu=\partial \iota / \partial \jmath=$ $\varepsilon / \varepsilon=1$ on $R^{\beta}$. Take a complement $K$ such that $H=R^{\beta} \oplus K$ and extend $\mu$ to $H$ by letting $\mu(K)=1$. Then $\mu$ j coincides with $\iota$ on $R^{\beta}$ as desired. Define a character $\chi_{\gamma}$ of $H$ by $\chi_{\gamma}(\alpha):=(-1)^{\langle\gamma, \alpha\rangle}$ for $\alpha \in H$ and extend to a character $\tilde{\chi}_{\gamma ; \iota}$ of $\pi_{\mathbb{C}^{*}}^{-1}(H)$ by $\tilde{\chi}_{\gamma ; \iota}(\alpha, \iota(\alpha) u):=\chi_{\gamma}(\alpha) u$ for $\alpha \in H$ and $u \in \mathbb{C}^{*}$. Note that $\tilde{\chi}_{\gamma ; \iota}$ also defines a linear character on $\tilde{H}$. Moreover, every character $\varphi$ of $\tilde{H}$ such that $\varphi(0,-1)=-1$ is of the form $\tilde{\chi}_{\gamma ; \iota}$ for some $\gamma \in \mathbb{Z}_{2}^{n}$. Then by Theorem 4.3 , the pair $(\beta, \gamma)$ determines an irreducible $V_{C^{-}}$module

$$
M_{C}(\beta, \gamma ; \iota):=\operatorname{Ind}_{V_{H}}^{V_{C}}\left(L\left(h_{\beta, \gamma}\right), \tilde{\chi}_{\gamma ; \iota}\right) .
$$

Note that if $C$ is self-orthogonal and $\operatorname{supp}(C) \subset \operatorname{supp}(\beta)$, then $M_{C}(\beta, \gamma ; \iota) \simeq L\left(h_{\beta, \gamma}\right)$ as a $V(0)$-module. If $\beta=0$, then $H=0$ and $\iota$ is trivial. We shall simply denote $M_{C}(0, \gamma ; \iota)$ by $M_{C}(0, \gamma)$. It is also obvious that

$$
M_{C}(0, \gamma)=\bigoplus_{\alpha=\left(\alpha_{1}, \ldots, \alpha_{n}\right) \in C+\gamma} L\left(1 / 2, \alpha_{1} / 2\right) \otimes \cdots \otimes L\left(1 / 2, \alpha_{n} / 2\right)
$$

This module is called a coset module in $\mathrm{M}^{4}$. We sometimes denote $M_{C}(0, \gamma)$ by $V_{C+\gamma}$, also.

We shall review some basic properties of $M_{C}(\beta, \gamma ; \iota)$.

Lemma 4.5. ([DGL]) The module structure of $M_{C}(\beta, \gamma ; \iota)$ is independent of the choice of the maximal self-orthogonal subcode $H$ of $C_{\beta}$ and the choice of the extension of $\iota$ from $R^{\beta}$ to $H$.

Proof: Let $H^{\prime}$ be a maximal self-orthogonal subcode of $C_{\beta}$ and $\iota^{\prime}: H^{\prime} \rightarrow \mathbb{C}^{*}$ an extension of $\iota$ to $H^{\prime}$ such that the section map $H^{\prime} \ni \alpha \mapsto\left(\alpha, \iota^{\prime}(\alpha)\right) \in \pi_{\mathbb{C}^{*}}^{-1}\left(H^{\prime}\right)$ is a group homomorphism. Define a linear character $\tilde{\chi}_{\gamma ; \iota^{\prime}}^{\prime}$ of $\pi_{\mathbb{C}^{*}}^{-1}\left(H^{\prime}\right)$ by $\tilde{\chi}_{\gamma ; \iota^{\prime}}^{\prime}\left(\alpha, \iota^{\prime}(\alpha) u\right):=(-1)^{\langle\gamma, \alpha\rangle} u$ for $\alpha \in H^{\prime}$ and $u \in \mathbb{C}^{*}$. Then $\tilde{\chi}_{\gamma ; \iota^{\prime}}^{\prime}$ is also a linear character of the preimage $\tilde{H}^{\prime}$ of $H^{\prime}$ in (4.3). We shall show that

$$
\operatorname{Ind}_{V_{H^{\prime}}}^{V_{C}}\left(L\left(h_{\beta, \gamma}\right), \tilde{\chi}_{\gamma ; \iota^{\prime}}\right) \simeq \operatorname{Ind}_{V_{H}}^{V_{C}}\left(L\left(h_{\beta, \gamma}\right), \tilde{\chi}_{\gamma, \iota}\right) .
$$

\footnotetext{
${ }^{4}$ This name has nothing to do with so-called the coset construction (cf. [FZ, GKO]) of a commutant subalgebra.
} 
For this, it suffices to show that $\operatorname{Ind}_{\tilde{H}}^{\tilde{C}_{\beta}} \tilde{\chi}_{\gamma ; \iota} \simeq \operatorname{Ind}_{\tilde{H}^{\prime}}^{\tilde{C}_{\beta}} \tilde{\chi}_{\gamma ; \iota^{\prime}}^{\prime}$ by Theorem 4.2 and the construction of induced modules. By definition, it is clear that $\left.\tilde{\chi}_{\gamma ; \iota}\right|_{R^{\beta}}=\left.\tilde{\chi}_{\gamma ; \iota^{\prime}}^{\prime}\right|_{R^{\beta}}$. For simplicity, we denote it by $\lambda$.

Let $K$ be a compliment of $R^{\beta}$ in $H+H^{\prime}$ and take $D$ be a complement of $R^{\beta}$ in $C_{\beta}$ such that $K \subset D$. Then $H=R^{\beta} \oplus(H \cap K), H^{\prime}=R^{\beta} \oplus\left(H^{\prime} \cap K\right)$ and $\tilde{C}_{\beta} \simeq \tilde{R}^{\beta} *_{\{ \pm 1\}} \tilde{D}$. It is obvious that both $H_{1}=H \cap K$ and $H_{2}=H^{\prime} \cap K$ are maximal self-orthogonal subcodes of $D$. Therefore, by Proposition 4.1, we have $\left.\operatorname{Ind}_{\tilde{H}}^{\tilde{C}_{\beta}} \tilde{\chi}_{\gamma ; \iota} \simeq \lambda \otimes_{\mathbb{C}} \operatorname{Ind}_{\tilde{H}_{1}}^{\tilde{D}} \tilde{\chi}_{\gamma ; \iota}\right|_{\tilde{H}_{1}}$ and $\left.\operatorname{Ind}_{\tilde{H}^{\prime}}^{\tilde{C}_{\beta}} \tilde{\chi}_{\gamma ; l^{\prime}}^{\prime} \simeq \lambda \otimes_{\mathbb{C}} \operatorname{Ind}_{\tilde{H}_{2}}^{\tilde{D}} \tilde{\chi}_{\gamma ; \iota^{\prime}}^{\prime}\right|_{\tilde{H}_{2}}$. Since there is only one linear representation of $\tilde{D}$ such that $(0,-1)$ acts non-trivially, we have $\operatorname{Ind}_{\tilde{H}_{1}}^{\tilde{D}} \tilde{\chi}_{\gamma ; \iota} \simeq \operatorname{Ind}_{\tilde{H}_{2}}^{\tilde{D}} \tilde{\chi}_{\gamma ; \iota^{\prime}}^{\prime}$ and $\operatorname{Ind}_{\tilde{H}_{\beta}}^{\tilde{C}_{\beta}} \tilde{\chi}_{\gamma ; \iota} \simeq \operatorname{Ind}_{\tilde{H}^{\prime}}^{\tilde{C}_{\beta}} \tilde{\chi}_{\gamma ; \iota}^{\prime}$ as desired

Remark 4.6. If we choose another map $\iota^{\prime}: R^{\beta} \rightarrow \mathbb{C}^{*}$ such that the section map $R^{\beta} \ni$ $\alpha \mapsto\left(\alpha, \iota^{\prime}(\alpha)\right) \in \pi_{\mathbb{C}^{*}}^{-1}\left(R^{\beta}\right)$ is a group homomorphism, then $\iota / \iota^{\prime}$ is a linear character of $R^{\beta}$. Thus, there exists $\xi \in\left(\mathbb{Z}_{2}^{n}\right)_{\beta}$ such that $\iota(\alpha) / \iota^{\prime}(\alpha)=(-1)^{\langle\alpha, \xi\rangle}$ for $\alpha \in R^{\beta}$. Hence, for any $\alpha \in R^{\beta}$ and $u \in \mathbb{C}^{*}$, we have

$$
\begin{aligned}
\tilde{\chi}_{\gamma ; \iota^{\prime}}(\alpha, \iota(\alpha) u) & =\tilde{\chi}_{\gamma ; \iota^{\prime}}\left(\alpha,(-1)^{\langle\alpha, \xi\rangle} \iota^{\prime}(\alpha) u\right)=(-1)^{\langle\alpha, \xi\rangle} \cdot(-1)^{\langle\alpha, \gamma\rangle} u \\
& =(-1)^{\langle\alpha, \gamma+\xi\rangle} u=\tilde{\chi}_{\gamma+\xi ; \iota}(\alpha, \iota(\alpha) u) .
\end{aligned}
$$

Hence, $\tilde{\chi}_{\gamma ; \iota^{\prime}}=\tilde{\chi}_{\gamma+\xi ; \iota}$ on $\tilde{R}^{\beta}$ and we have $M_{C}\left(\beta, \gamma ; \iota^{\prime}\right) \simeq M_{C}(\beta, \gamma+\xi ; \iota)$.

Similarly, one can show the following by considering linear characters of $\tilde{H}$.

Lemma 4.7. ([DGL]) Let $\beta, \beta^{\prime} \in C^{\perp}$ and $\gamma, \gamma^{\prime} \in \mathbb{Z}_{2}^{n}$. Then the irreducible $V_{C}$-modules $M_{C}(\beta, \gamma ; \iota)$ and $M_{C}\left(\beta^{\prime}, \gamma^{\prime} ; \iota\right)$ are isomorphic if and only if

$$
\beta=\beta^{\prime} \quad \text { and } \quad \gamma+\gamma^{\prime} \in C+H^{\perp_{\beta}}
$$

where $H$ is a maximal self-orthogonal subcode of $C_{\beta}$ and $H^{\perp_{\beta}}=\left\{\alpha \in \mathbb{Z}_{2}^{n} \mid \operatorname{supp}(\alpha) \subset\right.$ $\operatorname{supp}(\beta)$ and $\langle\alpha, \delta\rangle=0$ for all $\delta \in H\}$.

Proof: Assume that $M_{C}(\beta, \gamma ; \iota) \simeq M_{C}\left(\beta^{\prime}, \gamma^{\prime} ; \iota\right)$. Then clearly $\beta=\beta^{\prime}$ by $1 / 16$-word decompositions. It is also obvious from the definition of $M_{C}(\beta, \gamma ; \iota)$ that $M_{C}(\beta, \gamma ; \iota) \simeq$ $M_{C}(\beta, \gamma+\delta ; \iota)$ for any $\delta \in H^{\perp_{\beta}}$. Let $\left\{\alpha_{1}, \ldots, \alpha_{r}\right\}$ and $\left\{\delta_{1}, \ldots, \delta_{s}\right\}$ be transversals for $C_{\beta}$ in $C$ and $H$ in $C_{\beta}$, respectively. Then by definition we have a decomposition

$$
M_{C}(\beta, \gamma ; \iota)=\bigoplus_{i=1}^{r} \bigoplus_{j=1}^{s}\left(V\left(\alpha_{i}\right) \underset{V(0)}{\otimes} L\left(h_{\beta, \gamma}\right)\right) \underset{\mathbb{C}}{\otimes}\left(e^{\alpha_{i}+\delta_{j}} \underset{\mathbb{C}^{\varepsilon}[H]}{\otimes} \tilde{\chi}_{\gamma ; \iota}\right) .
$$

It follows from (4.6) that

$$
\left(V\left(\alpha_{i}\right) \underset{V(0)}{\otimes} L\left(h_{\beta, \gamma}\right)\right) \underset{\mathbb{C}}{\otimes}\left(e^{\alpha_{i}+\delta_{j}} \underset{\mathbb{C}^{\varepsilon}[H]}{\otimes} \tilde{\chi}_{\gamma ; \iota}\right) \simeq M_{H}\left(\beta, \gamma+\alpha_{i}+\delta_{j} ; \iota\right)
$$


as a $V_{H}$-submodule. Therefore, we have the following decompositions:

$$
\begin{aligned}
& M_{C}(\beta, \gamma ; \iota)=\bigoplus_{\delta+H \in C / H} M_{H}(\beta, \gamma+\delta ; \iota), \\
& M_{C}\left(\beta, \gamma^{\prime} ; \iota\right)=\bigoplus_{\delta+H \in C / H} M_{H}\left(\beta, \gamma^{\prime}+\delta ; \iota\right) .
\end{aligned}
$$

Since $H=C_{\beta} \cap H^{\perp_{\beta}}$ by the maximality of $H$, all $M_{H}(\beta, \gamma+\delta ; \iota), \delta \in C / H$, are inequivalent

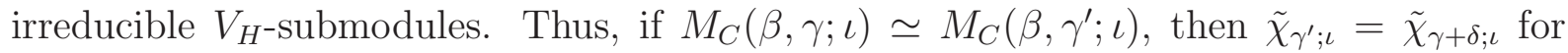
some $\delta \in C$. This is possible if and only if $\gamma+\gamma^{\prime} \in C+H^{\perp_{\beta}}$. Conversely, if $\gamma+\gamma^{\prime} \in C+H^{\perp_{\beta}}$,

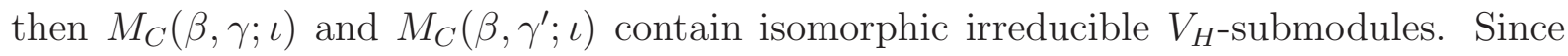
$V_{C}$-module structures on $M_{C}(\beta, \gamma ; \iota)$ and $M_{C}\left(\beta, \gamma^{\prime} ; \iota\right)$ are uniquely determined by their $V_{H}$-module structures, they are isomorphic.

In the proof above, we have shown the following useful fact.

Corollary 4.8. Let $M_{C}(\beta, \gamma ; \iota)$ be an irreducible $V_{C}$-module. Let $H$ be a maximal selforthogonal subcode of $C_{\beta}$. Then as a $V_{H}$-module,

$$
M_{C}(\beta, \gamma ; \iota)=\bigoplus_{\delta+H \in C / H} M_{H}(\beta, \gamma+\delta ; \iota) .
$$

In particular, every irreducible $V_{H}$-submodule of $M_{C}(\beta, \gamma ; \iota)$ is multiplicity-free.

Lemma 4.9. Let $R$ be the radical of $C_{\beta}$ with respect to the standard bilinear form and $H$ a maximal self-orthogonal subcode of $C_{\beta}$. Then $C_{\beta}+H^{\perp_{\beta}}=R^{\perp_{\beta}}$ and hence the code $C+H^{\perp_{\beta}}=C+R^{\perp_{\beta}}$ is again independent of the choice of the maximal self-orthogonal subcode $H$.

Proof: Since $R \subset H$, we have $H^{\perp_{\beta}} \subset R^{\perp_{\beta}}$. By definition, it is also clear that $C_{\beta} \subset R^{\perp_{\beta}}$ and we have $C_{\beta}+H^{\perp_{\beta}} \subset R^{\perp_{\beta}}$. Since the bilinear form $\langle\cdot, \cdot\rangle$ restricted on the quotient space $C_{\beta} / R$ is non-degenerate and $H / R$ is a maximal self-orthogonal subspace, we have $\operatorname{dim} C_{\beta} / R=2 \operatorname{dim} H / R$ and hence $\operatorname{dim} C_{\beta}=2 \operatorname{dim} H-\operatorname{dim} R$. On the other hand,

$$
\begin{aligned}
\operatorname{dim}\left(C_{\beta}+H^{\perp_{\beta}}\right) & =\operatorname{dim} C_{\beta}+\operatorname{dim} H^{\perp_{\beta}}-\operatorname{dim}\left(C_{\beta} \cap H^{\perp_{\beta}}\right) \\
& =\operatorname{dim} C_{\beta}+\operatorname{wt} \beta-2 \operatorname{dim} H=\operatorname{wt} \beta-\operatorname{dim} R=\operatorname{dim} R^{\perp_{\beta}} .
\end{aligned}
$$

Thus, we have $C_{\beta}+H^{\perp_{\beta}}=R^{\perp_{\beta}}$

\subsection{Dual module}

We shall determine the structure of the dual module of a $V_{C}$-module $M_{C}(\beta, \gamma ; \iota)$. Recall that the dual module of a $V$-module $M=\oplus_{n \in \mathbb{N}} M_{n+h}$ is defined to be its restricted dual $M^{*}=\oplus_{n \in \mathbb{N}} M_{n+h}^{*}$ equipped with a vertex operator $Y_{M}^{*}(\cdot, z)$ defined by (2.2). 
First, we consider the case when the code is self-orthogonal. Let $H$ be a self-orthogonal code of $\mathbb{Z}_{2}^{n}$. In this case, one can define a character $\varphi$ of $H$ by $\varphi(\alpha)=(-1)^{\mathrm{wt}(\alpha) / 2}$ for $\alpha \in H$. So there exists a codeword $\kappa \in \mathbb{Z}_{2}^{n}$ such that $\varphi(\alpha)=(-1)^{\langle\kappa, \alpha\rangle}$ for all $\alpha \in H$.

Lemma 4.10. Let $H \subset \mathbb{Z}_{2}^{n}$ be a self-orthogonal code. For any $\gamma \in \mathbb{Z}_{2}^{n}$, the dual module of $M_{H}\left(\left(1^{n}\right), \gamma ; \iota\right)$ is isomorphic to $M_{H}\left(\left(1^{n}\right), \gamma+\kappa ; \iota\right)$, where $\kappa \in \mathbb{Z}_{2}^{n}$ is given by $(-1)^{\langle\kappa, \alpha\rangle}=$ $(-1)^{\mathrm{wt}(\alpha) / 2}$ for all $\alpha \in H$.

Proof: By assumption, $M_{H}\left(\left(1^{n}\right), \gamma ; \iota\right)=L(1 / 2,1 / 16)^{\otimes n} \otimes \tilde{\chi}_{\gamma ; \iota}$ is an irreducible $V(0)=$ $L(1 / 2,0)^{\otimes n}$-module. Therefore,

$$
M_{H}\left(\left(1^{n}\right), \gamma ; \iota\right)^{*} \simeq M_{H}\left(\left(1^{n}\right), \gamma^{\prime} ; \iota\right) \quad \text { for some } \gamma^{\prime} \in \mathbb{Z}_{2}^{n}
$$

Since $L(1 / 2,1 / 16)^{\otimes n}$ is a self-dual $V(0)$-module, we have a $V(0)$ - isomorphism $f: M \rightarrow M^{*}$. Let $Y(\cdot, z)$ and $Y^{*}(\cdot, z)$ be the vertex operators on $M$ and $M^{*}$, respectively. For $\alpha \in H$, let $x^{\alpha} \in V(\alpha)$ be a non-zero highest weight vector of weight $\operatorname{wt}(\alpha) / 2$. Then $Y^{*}\left(x^{\alpha}, z\right) f$ and $f Y\left(x^{\alpha}, z\right)$ are $V(0)$-intertwining operators of type

$$
V(\alpha) \times M_{H}\left(\left(1^{n}\right), \gamma ; \iota\right) \rightarrow M_{H}\left(\left(1^{n}\right), \gamma ; \iota\right)^{*} .
$$

Since the space of $V(0)$-intertwining operators of this type is one-dimensional, there exists a scalar $\lambda_{\alpha} \in \mathbb{C}^{*}$ such that $Y^{*}\left(x^{\alpha}, z\right) f=\lambda_{\alpha} f Y\left(x^{\alpha}, z\right)$. Let $v$ be a non-zero highest weight vector of $M_{H}\left(\left(1^{n}\right), \gamma ; \iota\right)$. Then by (2.2), one has

$$
\begin{aligned}
\left\langle Y^{*}\left(x^{\alpha}, z\right) f v, v\right\rangle & =(-1)^{\mathrm{wt}(\alpha) / 2} z^{-\operatorname{wt}(\alpha)}\left\langle f v, Y\left(x^{\alpha}, z^{-1}\right) v\right\rangle \\
& =(-1)^{\operatorname{wt}(\alpha) / 2} z^{-\operatorname{wt}(\alpha) / 2}\left\langle f v, x_{(\operatorname{wt}(\alpha) / 2-1)}^{\alpha} v\right\rangle .
\end{aligned}
$$

On the other hand,

$$
\begin{aligned}
\left\langle Y^{*}\left(x^{\alpha}, z\right) f v, v\right\rangle & =\lambda_{\alpha}\left\langle f Y\left(x^{\alpha}, z\right) v, v\right\rangle \\
& =\lambda_{\alpha} z^{-\operatorname{wt}(\alpha) / 2}\left\langle f x_{(\operatorname{wt}(\alpha) / 2-1)}^{\alpha} v, v\right\rangle .
\end{aligned}
$$

Since $x_{(\mathrm{wt}(\alpha) / 2-1)}^{\alpha} v=t v$ for some $t \in \mathbb{C}^{*}$ and $\langle f v, v\rangle \neq 0$, we have $\lambda_{\alpha}=(-1)^{\mathrm{wt}(\alpha) / 2}=$ $(-1)^{\langle\kappa, \alpha\rangle}$. Therefore, by considering the linear character associated to $M_{H}\left(\left(1^{n}\right), \gamma ; \iota\right)^{*}$, we see that $M_{H}\left(\left(1^{n}\right), \gamma ; \iota\right)^{*} \simeq M_{H}\left(\left(1^{n}\right), \gamma+\kappa ; \iota\right)$.

Proposition 4.11. Let $C$ be an even linear code, $\beta \in C^{\perp}$ and $\gamma \in \mathbb{Z}_{2}^{n}$. Let $H$ be a maximal self-orthogonal subcode of $C_{\beta}$. Then the dual module $M_{C}(\beta, \gamma ; \iota)^{*}$ is isomorphic to $M_{C}\left(\beta, \gamma+\kappa_{H} ; \iota\right)$ where $\kappa_{H} \in \mathbb{Z}_{2}^{n}$ is such that $\operatorname{supp}\left(\kappa_{H}\right) \subset \operatorname{supp}(\beta)$ and $(-1)^{\left\langle\kappa_{H}, \alpha\right\rangle}=$ $(-1)^{\mathrm{wt}(\alpha) / 2}$ for all $\alpha \in H$.

Proof: By Corollary 4.8, $M_{C}(\beta, \gamma ; \iota)$ contains a $V_{H^{-}}$-submodule

$$
M_{H}(\beta, \gamma ; \iota) \simeq L\left(h_{\beta, \gamma}\right) \otimes \tilde{\chi}_{\gamma ; \iota}
$$


By the previous lemma, the dual module $M_{C}(\beta, \gamma ; \iota)^{*}$ contains a $V_{H^{-}}$-submodule isomorphic to

$$
M_{H}(\beta, \gamma ; \iota)^{*} \simeq M_{H}\left(\beta, \gamma+\kappa_{H} ; \iota\right)=L\left(h_{\beta, \gamma}\right) \otimes \tilde{\chi}_{\gamma+\kappa_{H} ; \iota}
$$

Therefore, by the structure of irreducible $V_{C}$-modules,

$$
M_{C}(\beta, \gamma ; \iota)^{*} \simeq \operatorname{Ind}_{V_{H}}^{V_{C}}\left(L\left(h_{\beta, \gamma}\right), \tilde{\chi}_{\gamma+\kappa_{H} ; \iota}\right)
$$

and hence $M_{C}(\beta, \gamma ; \iota)^{*} \simeq M_{C}\left(\beta, \gamma+\kappa_{H} ; \iota\right)$.

As an immediate corollary, we have:

Corollary 4.12. With reference to the proposition above, $M_{C}(\beta, \gamma ; \iota)$ is self-dual if and only if $\kappa_{H} \in C$. In particular, $M_{C}(0, \gamma)$ is self-dual for all $\gamma \in \mathbb{Z}_{2}^{n}$.

\subsection{Fusion rules}

We shall compute the fusion rules among some irreducible $V_{C}$-modules. First, we recall a result from [M2] which is a direct consequence of Proposition 2.5 .

Lemma 4.13. ([M2]) For $\alpha, \beta \in \mathbb{Z}_{2}^{n}, M_{C}(0, \alpha) \bigotimes_{V_{C}} M_{C}(0, \beta)=M_{C}(0, \alpha+\beta)$.

By the lemma above, we see that $M_{C}(0, \alpha) \bigotimes_{V_{C}} M_{C}(0, \alpha)=M_{C}(0,0) \simeq V_{C}$. Therefore, all $M_{C}(0, \alpha), \alpha \in \mathbb{Z}_{2}^{n}$, are simple current $V_{C}$-modules by Corollary 2.2. It also follows that $M_{C}(0, \alpha) \bigotimes_{V_{C}} M_{C}(\beta, \gamma ; \iota)$ is an irreducible $V_{C}$-module with the $1 / 16$-word $\beta$. The corresponding fusion rules are also computed by Miyamoto [M3] in the case $\operatorname{supp}(\alpha) \subset$ $\operatorname{supp}(\beta)$.

Lemma 4.14. ([M3] $)$ Let $\alpha, \beta, \gamma \in \mathbb{Z}_{2}^{n}$ with $\beta \in C^{\perp}$. Then

$$
M_{C}(0, \alpha) \underset{V_{C}}{\bigotimes} M_{C}(\beta, \gamma ; \iota)=M_{C}(\beta, \alpha+\gamma ; \iota)
$$

Moreover, the difference of the top weight of $M_{C}(\beta, \gamma ; \iota)$ and the top weight of $M_{C}(\beta, \alpha+$ $\gamma ; \iota)$ is congruent to $\langle\alpha, \alpha+\beta\rangle / 2 \operatorname{modulo} \mathbb{Z}$.

Proof: The assertion is proved in Lemma 3.27 of [M3] in the case $\operatorname{supp}(\alpha) \subset \operatorname{supp}(\beta)$. We generalize his argument to obtain the desired fusion rule. Since $M_{C}(0, \alpha)$ is a simple current $V_{C}$-module, we know that there exists $\gamma^{\prime} \in \mathbb{Z}_{2}^{n}$ such that

$$
M_{C}(0, \alpha) \underset{V_{C}}{\bigotimes} M_{C}(\beta, \gamma ; \iota) \simeq M_{C}\left(\beta, \gamma^{\prime} ; \iota\right)
$$

Therefore, if we can construct a non-zero $V_{C}$-intertwining operator of type $M_{C}(0, \alpha) \times$ $M_{C}(\beta, \gamma ; \iota) \rightarrow M_{C}(\beta, \alpha+\gamma ; \iota)$, then we are done. To do this, we have to extend $V_{C}$ to a larger algebra. The case $\alpha \in C$ is trivial so that we assume that $\alpha \notin C$. Set 
$C^{\prime}=C \sqcup(C+\alpha)$. We can define a simple vertex operator (super)algebra structure on the space $V_{C^{\prime}}=V_{C} \oplus V_{C+\alpha}=M_{C}(0,0) \oplus M_{C}(0, \alpha)$. This is a VOA if $\alpha$ is even, and an SVOA if $\alpha$ is odd.

Set $H^{\prime}:=C_{\beta}^{\prime} \cap H^{\perp}$, which is the unique maximal subcode of $C_{\beta}^{\prime}$ containing $H$ such that its preimage $\tilde{H}^{\prime}$ is a maximal abelian subgroup of $\tilde{C}_{\beta}^{\prime}$ in the extension (4.3). We can take $\jmath: H^{\prime} \rightarrow \mathbb{C}^{*}$ such that $\left.\jmath\right|_{H}=\iota$ and the section map $H^{\prime} \ni \delta \mapsto(\delta, \jmath(\delta)) \in \pi_{\mathbb{C}^{*}}^{-1}\left(H^{\prime}\right)$ defines a group homomorphism. In the definition of the induced module $M_{C}(\beta, \gamma ; \iota)=$ $\operatorname{Ind}_{V_{H}}^{V_{C}} L\left(h_{\beta, \gamma}, \tilde{\chi}_{\gamma ; \iota}\right)$, if we use $\mathbb{C}^{\varepsilon}\left[C^{\prime}\right]$ instead of $\mathbb{C}^{\varepsilon}[C]$ and replace $\iota$ by $\jmath$, then we obtain an irreducible $V_{C^{\prime}}$-module

$$
M_{C^{\prime}}(\beta, \gamma ; \jmath):=\operatorname{Ind}_{V_{H^{\prime}}}^{V_{C^{\prime}}}\left(L\left(h_{\beta, \gamma}, \tilde{\chi}_{\gamma, \jmath}\right)\right.
$$

which contains $M_{C}(\beta, \gamma ; \iota)=\operatorname{Ind}_{V_{H}}^{V_{C}}\left(L\left(h_{\beta, \gamma}, \tilde{\chi}_{\gamma, \jmath}\right)\right.$ as a $V_{C}$-submodule. The induced module $M_{C^{\prime}}(\beta, \gamma ; \jmath)$ is an untwisted $V_{C^{\prime}}$-module if $\langle\alpha, \beta\rangle=0$ and otherwise it is a $\mathbb{Z}_{2}$-twisted $V_{C^{\prime}}$-module (cf. [Y2]). Nevertheless, the subspace $M=V_{C+\alpha} \cdot M_{C}(\beta, \gamma ; \iota)$ of $M_{C^{\prime}}(\beta, \gamma ; \jmath)$ is an irreducible $V_{C}$-submodule. It follows from (4.6) and (4.7) that there exists an irreducible $V_{H}$-submodule of $M$ isomorphic to $M_{H}\left(\beta, \alpha+\gamma ;\left.\jmath\right|_{H}\right)$. Then $M \simeq M_{C}\left(\beta, \alpha+\gamma ;\left.\jmath\right|_{H}\right)$ by the structure of an irreducible $V_{C}$-module. Since $M \simeq M_{C}(0, \alpha) \bigotimes_{V_{C}} M_{C}(\beta, \gamma ; \iota)$, we obtain the desired fusion rule.

Since the $L(1 / 2,0)$-intertwining operators of types $L(1 / 2, h) \times L(1 / 2,1 / 16) \rightarrow L(1 / 2,1 / 16), h \in$ $\{0,1 / 2\}$, keep the top weights but the $L(1 / 2,0)$-intertwining operators of type $L(1 / 2,1 / 2) \times$ $L(1 / 2,0) \rightarrow L(1 / 2,1 / 2)$ and $L(1 / 2,1 / 2) \times L(1 / 2,1 / 2) \rightarrow L(1 / 2,0)$ change the top weights by $1 / 2$ or $-1 / 2$, the difference of top weights is as in the assertion.

By this lemma, we can compute the following fusion rule.

Proposition 4.15. Let $\beta \in C^{\perp}$ and $\gamma \in \mathbb{Z}_{2}^{n}$. Let $H$ be a maximal self-orthogonal subcode of $C_{\beta}$. Then

$$
M_{C}(\beta, \gamma ; \iota) \underset{V_{C}}{\bigotimes} M_{C}(\beta, \gamma ; \iota)^{*}=\sum_{\delta+C \in C+H^{\perp_{\beta}}} M_{C}(0, \delta),
$$

where $\delta \in \mathbb{Z}_{2}^{n}$ runs over a transversal for $C$ in $C+H^{\perp_{\beta}}$.

Proof: It follows from the 1/16-word decomposition that the fusion product

$$
M_{C}(\beta, \gamma ; \iota) \underset{V_{C}}{\bigotimes} M_{C}(\beta, \gamma ; \iota)^{*}
$$

contains only modules of type $M_{C}(0, \delta)$. Now assume that $\left(\begin{array}{c}M_{C}(0, \delta) \\ \left.M_{C}(\beta, \gamma ; \iota) \stackrel{M_{C}(\beta, \gamma ; \iota)^{*}}{ }\right)_{V_{C}}\end{array} \neq 0\right.$. Then by the symmetry of fusion rules, we have

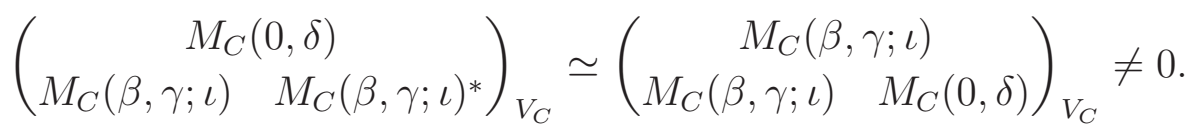

Since $M_{C}(\beta, \gamma ; \iota) \bigotimes_{V_{C}} M_{C}(0, \delta)=M_{C}(\beta, \gamma+\delta ; \iota)$ by the previous lemma, this is possible if and only if $\delta \in C+H^{\perp_{\beta}}$ by Lemma 4.7. Therefore, we have the fusion rule as stated. 
By the lemma above, we introduce the following definition.

Definition 4.16. Let $\beta \in \mathbb{Z}_{2}^{n}$ and $H$ a subcode with $\operatorname{supp}(H) \subset \operatorname{supp}(\beta) . H$ is said to be self-dual with respect to $\beta$ if $H=H^{\perp_{\beta}}$.

Remark 4.17. Note that if $H$ is a self-dual subcode of $C_{\beta}$ w.r.t. $\beta$ then $C+H^{\perp_{\beta}}=C$.

By Corollary 2.2 and Proposition 4.15, we have

Corollary 4.18. $M_{C}(\beta, \gamma ; \iota)$ is a simple current module if and only if $C_{\beta}$ contains a self-dual subcode w.r.t. $\beta$.

Remark 4.19. Now suppose $M_{C}(\beta, 0 ; \iota) \bigotimes_{V_{C}} M_{C}(\beta, 0 ; \iota)^{*}=\sum_{i=1}^{p} M_{C}\left(0, \delta_{i}\right)$. Let $H$ be a maximal self-orthogonal subcode of $C_{\beta}$, and let $\kappa_{H} \in\left(\mathbb{Z}_{2}^{n}\right)_{\beta}$ such that $\left\langle\kappa_{H}, \alpha\right\rangle=\langle\alpha, \alpha\rangle / 2$ $\bmod 2$ for all $\alpha \in H$ as in Proposition 4.11. Then

$$
M_{C}(\beta, 0 ; \iota)^{*}=M_{C}\left(\beta, \kappa_{H} ; \iota\right)=M_{C}\left(0, \kappa_{H}\right) \underset{V_{C}}{\bigotimes} M_{C}(\beta, 0 ; \iota)
$$

and thus $M_{C}(\beta, 0 ; \iota)=M_{C}\left(0, \kappa_{H}\right) \bigotimes_{V_{C}} M_{C}(\beta, 0 ; \iota)^{*}$. Using this, we can compute the following fusion rule:

$$
\begin{aligned}
& M_{C}\left(\beta, \gamma_{1} ; \iota\right) \underset{V_{C}}{\otimes} M_{C}\left(\beta, \gamma_{2} ; \iota\right) \\
& =\left\{M_{C}\left(0, \gamma_{1}\right) \underset{V_{C}}{\otimes} M_{C}(\beta, 0 ; \iota)\right\} \underset{V_{C}}{\otimes}\left\{M_{C}\left(0, \gamma_{2}\right) \underset{V_{C}}{\otimes} M_{C}(\beta, 0 ; \iota)\right\} \\
& =M_{C}\left(0, \gamma_{1}+\gamma_{2}\right) \underset{V_{C}}{\otimes} M_{C}(\beta, 0 ; \iota) \underset{V_{C}}{\otimes} M_{C}(\beta, 0 ; \iota) \\
& =M_{C}\left(0, \gamma_{1}+\gamma_{2}\right) \underset{V_{C}}{\otimes} M_{C}(\beta, 0 ; \iota) \underset{V_{C}}{\otimes}\left\{M_{C}\left(0, \kappa_{H}\right) \underset{V_{C}}{\otimes} M_{C}(\beta, 0 ; \iota)^{*}\right\} \\
& =M_{C}\left(0, \gamma_{1}+\gamma_{2}+\kappa_{H}\right) \underset{V_{C}}{\otimes} M_{C}(\beta, 0 ; \iota) \underset{V_{C}}{\otimes} M_{C}(\beta, 0 ; \iota)^{*} \\
& =M_{C}\left(0, \gamma_{1}+\gamma_{2}+\kappa_{H}\right) \underset{V_{C}}{\otimes}\left\{\sum_{i=1}^{p} M_{C}\left(0, \delta_{i}\right)\right\} \\
& =\sum_{i=1}^{p} M_{C}\left(0, \gamma_{1}+\gamma_{2}+\kappa_{H}+\delta_{i}\right) .
\end{aligned}
$$

\section{Structure of framed VOAs}

We shall prove that every framed VOA is a simple current extension of a code VOA. This result has many fruitful consequences. For example, the irreducible representations of a framed VOA can be determined by a notion of induced modules. Another interesting result is the conditions on possible structure codes of holomorphic framed VOAs, namely we obtain a necessary and sufficient condition for a pair of codes $(C, D)$ to be the structure codes of some holomorphic framed VOAs in Theorem 5.17. 


\subsection{Simple current structure}

In this subsection we discuss how a code VOA can be extended to a framed VOA. First, we give a construction of a non-trivial simple current extension.

Lemma 5.1. Let $C$ be an even linear subcode of $\mathbb{Z}_{2}^{n}$ and $\beta \in C^{\perp}$ a non-zero codeword. Let $\gamma \in \mathbb{Z}_{2}^{n}$ be a binary codeword such that the irreducible $V_{C}$-module $M_{C}(\beta, \gamma ; \iota)$ has an integral top weight. If $C_{\beta}$ contains a doubly even self-dual subcode w.r.t. $\beta$, then there exists a unique structure of a framed VOA on $V_{C} \oplus M_{C}(\beta, \gamma ; \iota)$ which forms a $\mathbb{Z}_{2}$-graded simple current extension of $V_{C}$.

Proof: Let $H$ be a doubly even self-dual subcode of $C_{\beta}$ w.r.t. $\beta$. By Proposition 4.11, $M_{C}(\beta, \gamma ; \iota)$ is self-dual, and by Corollary $4.18, M_{C}(\beta, \gamma ; \iota)$ is a simple current $V_{C}$-module. By Corollary $4.8, M_{C}(\beta, \gamma ; \iota)$ has a $V_{H}$-module structure

$$
M_{C}(\beta, \gamma ; \iota)=\bigoplus_{\delta+H \in C / H} M_{H}(\beta, \gamma+\delta ; \iota)
$$

where all irreducible $V_{H}$-submodules $M_{H}(\beta, \gamma+\delta ; \iota)$ are self-dual by Proposition 4.11, It is clear that a $V_{C}$-invariant bilinear form on $M_{C}(\beta, \gamma ; \iota)$ induces a non-degenerate $V_{H}$-invariant bilinear form on $M_{H}(\beta, \gamma+\delta ; \iota)$. It is shown in Li1 that a $V_{C}$-invariant bilinear form on $M_{C}(\beta, \gamma ; \iota)$ is either symmetric or skew-symmetric. Since the top level of $M_{H}(\beta, \gamma+\delta ; \iota)$ is one-dimensional, the $V_{C}$-invariant bilinear form on $M_{C}(\beta, \gamma ; \iota)$ must be symmetric. Therefore, $V_{C} \oplus M_{C}(\beta, \gamma ; \iota)$ forms a $\mathbb{Z}_{2}$-graded simple current extension of $V_{C}$ by Proposition 2.6.

Lemma 5.2. Let $\beta \in C^{\perp}$ and $\gamma \in \mathbb{Z}_{2}^{n}$ with $\beta \neq 0$. Assume that $V=V_{C} \oplus M_{C}(\beta, \gamma ; \iota)$ forms a framed VOA. Then there exists a maximal self-orthogonal subcode $K$ of $C_{\beta}$ which is doubly even.

Proof: Let $H$ be a maximal self-orthogonal subcode of $C_{\beta}$. If $H$ is doubly even, then we are done. So we assume that $H$ contains a codeword whose weight is congruent to 2 modulo 4. Since $V=V_{C} \oplus M_{C}(\beta, \gamma ; \iota)$ forms a simple VOA, $M_{C}(\beta, \gamma ; \iota)$ is a self-dual $V_{C}$-module. Therefore, by Corollary 4.12 , there exists a codeword $\kappa_{H} \in C_{\beta}$ such that $(-1)^{\left\langle\kappa_{H}, \alpha\right\rangle}=(-1)^{\mathrm{wt}(\alpha) / 2}$ for all $\alpha \in H$. By Corollary 4.8, $M_{C}(\beta, \gamma ; \iota)$ has the following decomposition as a $V_{H}$-module:

$$
M_{C}(\beta, \gamma ; \iota)=\bigoplus_{\delta+H \in C / H} M_{H}(\beta, \gamma+\delta ; \iota)
$$

By our choice of $H, \kappa_{H} \notin H^{\perp_{\beta}}$ so that $M_{H}(\beta, \gamma ; \iota)$ and its dual $M_{H}\left(\beta, \gamma+\kappa_{H} ; \iota\right)$ are inequivalent irreducible $V_{H}$-submodules of $V$. We consider a sub VOA $U$ generated by 
$M_{H}(\beta, \gamma ; \iota) \oplus M_{H}\left(\beta, \gamma+\kappa_{H} ; \iota\right)$. By the fusion rule given in Proposition 4.15, $U$ has the following shape as a $V_{H}$-module:

$$
U=M_{H}(0,0) \oplus M_{H}\left(0, \kappa_{H}\right) \oplus M_{H}(\beta, \gamma ; \iota) \oplus M_{H}\left(\beta, \gamma+\kappa_{H} ; \iota\right)
$$

Note that $H=C \cap H^{\perp_{\beta}}$ by the maximality of $H$. Set $H^{\prime}:=H \sqcup\left(H+\kappa_{H}\right), H_{0}:=H \cap\left\langle\kappa_{H}\right\rangle^{\perp}$ and take any $\alpha^{\prime} \in H \backslash H_{0}$. Then

$$
H^{\prime}=H_{0} \sqcup\left(H_{0}+\alpha^{\prime}\right) \sqcup\left(H_{0}+\kappa_{H}\right) \sqcup\left(H_{0}+\alpha^{\prime}+\kappa_{H}\right) .
$$

We set $K:=H_{0} \sqcup\left(H_{0}+\kappa_{H}\right)$. It is clear that $U$ also possesses a symmetric invariant bilinear form which we shall denote by $\langle\cdot, \cdot\rangle_{U}$. Since $M_{H}(\beta, \gamma ; \iota)$ and $M_{H}\left(\beta, \gamma+\kappa_{H} ; \iota\right)$ are dual to each other, we have

$$
\left\langle M_{H}(\beta, \gamma ; \iota), M_{H}(\beta, \gamma ; \iota)\right\rangle_{U}=\left\langle M_{H}\left(\beta, \gamma+\kappa_{H} ; \iota\right), M_{H}\left(\beta, \gamma+\kappa_{H} ; \iota\right)\right\rangle_{U}=0
$$

By Lemma 4.7, $M_{H}(\beta, \gamma ; \iota)$ and $M_{H}\left(\beta, \gamma+\kappa_{H} ; \iota\right)$ are isomorphic irreducible $V_{H_{0}}$-modules and there exists a $V_{H_{0}}$-isomorphism $\varphi: M_{H}(\beta, \gamma ; \iota) \rightarrow M_{H}\left(\beta, \gamma+\kappa_{H} ; \iota\right)$. Let $u$ be a non-zero highest weight vector of $M_{H}(\beta, \gamma ; \iota)$. Since the top level of $M_{H}(\beta, \gamma ; \iota)$ is onedimensional, we may assume that $\langle u, \varphi(u)\rangle_{U}=1$. Now consider the decomposition (5.1) of $U$ with respect to a series of sub VOAs $V_{H_{0}} \subset V_{K} \subset V_{H^{\prime}}$ of $U$. It is clear that $M_{H}(0,0) \oplus M_{H}\left(0, \kappa_{H}\right)=M_{K}(0,0) \oplus M_{K}\left(0, \alpha^{\prime}\right)$. Therefore, there exists a decomposition of $U$ as a $V_{K}$-module

$$
U=M_{K}(0,0) \oplus M_{K}\left(0, \alpha^{\prime}\right) \oplus W
$$

with $W=M_{H}(\beta, \gamma ; \iota) \oplus M_{H}\left(\beta, \gamma+\kappa_{H} ; \iota\right)$. Let $W^{0}$ be an irreducible $V_{K^{-}}$submodule of $W$. Since $K$ is a self-orthogonal subcode of $C_{\beta}$, the top level of $W^{0}$ is one-dimensional. Let $v \in W^{0}$ be a non-zero highest weight vector. As we mentioned, $M_{H}(\beta, \gamma ; \iota)$ and $M_{H}\left(\beta, \gamma+\kappa_{H} ; \iota\right)$ are isomorphic $V_{H_{0}}$-submodules. But $M_{H}(\beta, \gamma ; \iota)$ and $M_{H}\left(\beta, \gamma+\kappa_{H} ; \iota\right)$ cannot form $V_{K}$-submodules by the fusion rule of $V_{H}$-modules. Therefore, we can write $v=c_{1} u+c_{2} \varphi(u)$ with $c_{1}, c_{2} \neq 0$. This shows that $\langle v, v\rangle_{U}=2 c_{1} c_{2} \neq 0$ so that $W^{0}$ is a self-dual $V_{K}$-submodule. Then $K$ is a doubly even code by Corollary 4.12. Since $|K|=|H|=2\left|H_{0}\right|, K$ is a maximal self-orthogonal subcode of $C_{\beta}$. Therefore, $C_{\beta}$ contains the desired subcode $K$.

We recall the following fact from the coding theory.

Theorem 5.3. ([McST]) Let $n$ be divisible by 8 and $H$ a doubly even code of $\mathbb{Z}_{2}^{n}$ containing the all-one vector $(11 \ldots 1) \in \mathbb{Z}_{2}^{n}$. Then there exists a doubly even self-dual code $H^{\prime}$ such that $H \subset H^{\prime}$.

Now we begin to prove that every framed VOA is a simple current extension of a code VOA. For this, it suffices to show the following proposition. 
Proposition 5.4. Let $\beta \in C^{\perp}$ and $\gamma \in \mathbb{Z}_{2}^{n}$. Assume that $V=V_{C} \oplus M_{C}(\beta, \gamma ; \iota)$ forms a framed VOA. Then $C_{\beta}$ contains a doubly even self-dual subcode w.r.t. $\beta$.

Proof: By Lemma 5.2, $C_{\beta}$ contains a maximal self-orthogonal subcode $H$ which is doubly even. By Corollary 4.8, $V$ has a decomposition

$$
V=\bigoplus_{\delta+H \in C / H} M_{H}(0, \delta) \oplus M_{H}(\beta, \gamma+\delta ; \iota)
$$

as a $V_{H}$-module. By the fusion rule in Proposition 4.15, the subspace

$$
U:=V_{H} \oplus M_{H}(\beta, \gamma ; \iota)
$$

forms a sub VOA of $V$, since $H=C_{\beta} \cap H^{\perp_{\beta}}$. If $H$ is not self-dual, then there exists a doubly even self-dual subcode $H^{\prime}$ of $\mathbb{Z}_{2}^{n}$ w.r.t. $\beta$ such that $H \cup(H+\beta) \subset H^{\prime}$ by Theorem 5.3. Note that the weight of $\beta$ is divisible by 8 since $M_{C}(\beta, \gamma ; \iota)$ has an integral top weight. Let us consider the code VOA $V_{H^{\prime}}$ associated to $H^{\prime}$. Since $H \subset H^{\prime}$, it is clear that $V_{H^{\prime}}$ contains $V_{H}$ as a sub VOA. We can also take a map $\jmath: H^{\prime} \rightarrow \mathbb{C}^{*}$ such that $\left.\jmath\right|_{H}=\iota$ and the section map $H^{\prime} \ni \alpha \mapsto(\alpha, \jmath(\alpha)) \in \pi_{\mathbb{C}^{*}}^{-1}\left(H^{\prime}\right)$ is a group homomorphism. By the structure theory in Theorem 4.3, we can define an irreducible $V_{H^{\prime}}$-module $M_{H^{\prime}}(\beta, \gamma ; \jmath)$ such that $\left.M_{H^{\prime}}(\beta, \gamma ; \jmath)\right|_{V_{H}} \simeq M_{H}(\beta, \gamma ; \iota)$ as a $V_{H^{-}}$-module. For simplicity, we shall denote $M_{H^{\prime}}(\beta, \gamma ; \jmath)$ by $W$. Since the top level of $W$ is one-dimensional, the $V_{H^{\prime}}$-invariant bilinear form on $W$ is symmetric. Therefore, by Proposition 2.5, we can define a framed VOA structure on

$$
U^{\prime}:=V_{H^{\prime}} \oplus W
$$

We denote the vertex operator on $U^{\prime}$ by $Y^{\prime}(\cdot, z)$. Now suppose $H$ is a proper subcode of $H^{\prime}$. Then

$$
V_{H^{\prime}}=\bigoplus_{\delta+H \in H^{\prime} / H} V_{\delta+H}, \quad V_{\delta+H}=M_{H}(0, \delta),
$$

as a $V_{H^{-}}$-module. Let $\pi_{\delta+H}: V_{H^{\prime}} \rightarrow V_{\delta+H}$ be the projection map. Then for $u, v \in W$, we have

$$
Y^{\prime}(u, z) v=\sum_{\delta+H \in H^{\prime} / H} \pi_{\delta+H} Y^{\prime}(u, z) v .
$$

Since the simple VOA structure is unique on $V_{H^{\prime}} \oplus W$, we may assume that $\pi_{H} Y^{\prime}(u, z) v=$ $Y_{V}(u, z) v$. Take any $\alpha \in H^{\prime} \backslash H$ and set $K:=H \sqcup(H+\alpha)$. We shall show the following claim:

Claim For $u, v \in W$, there exists $N=N(u, v) \in \mathbb{N}$ such that

$$
\left(z_{1}-z_{2}\right)^{N} Y^{\prime}\left(u, z_{1}\right) \pi_{H+\alpha} Y^{\prime}\left(v, z_{2}\right) w=\left(z_{1}-z_{2}\right)^{N} Y^{\prime}\left(v, z_{2}\right) \pi_{H+\alpha} Y^{\prime}\left(u, z_{1}\right) w
$$


for any $w \in W$.

Take any $a \in V_{H+\alpha}$. Since $U=V_{H} \oplus M_{H}(\beta, \gamma ; \iota)$ forms a framed VOA by assumption, there exists $N=N(u, v) \in \mathbb{N}$ such that

$$
\left(z_{1}-z_{2}\right)^{N} Y^{\prime}\left(u, z_{1}\right) \pi_{H} Y^{\prime}\left(v, z_{2}\right) w=\left(z_{1}-z_{2}\right)^{N} Y^{\prime}\left(v, z_{2}\right) \pi_{H} Y^{\prime}\left(u, z_{1}\right) w .
$$

Take a sufficiently large $k \in \mathbb{N}$. Then one has

$$
\begin{aligned}
& \left(z_{1}-z_{2}\right)^{N}\left(z_{0}-z_{1}\right)^{k}\left(z_{0}-z_{2}\right)^{k} Y^{\prime}\left(u, z_{1}\right) \pi_{H+\alpha} Y^{\prime}\left(v, z_{2}\right) Y^{\prime}\left(a, z_{0}\right) w \\
& =\left(z_{1}-z_{2}\right)^{N}\left(z_{0}-z_{1}\right)^{k}\left(z_{0}-z_{2}\right)^{k} Y^{\prime}\left(a, z_{0}\right) Y^{\prime}\left(u, z_{1}\right) \pi_{H} Y^{\prime}\left(v, z_{2}\right) w \\
& =\left(z_{1}-z_{2}\right)^{N}\left(z_{0}-z_{1}\right)^{k}\left(z_{0}-z_{2}\right)^{k} Y^{\prime}\left(a, z_{0}\right) Y^{\prime}\left(v, z_{2}\right) \pi_{H} Y^{\prime}\left(u, z_{1}\right) w \\
& =\left(z_{1}-z_{2}\right)^{N}\left(z_{0}-z_{1}\right)^{k}\left(z_{0}-z_{2}\right)^{k} Y^{\prime}\left(v, z_{2}\right) \pi_{H+\alpha} Y^{\prime}\left(u, z_{1}\right) Y^{\prime}\left(a, z_{0}\right) w .
\end{aligned}
$$

Since the expansions of both sides of the equations have only finitely many negative powers of $z_{0}$, we get

$$
\left(z_{1}-z_{2}\right)^{N} Y^{\prime}\left(u, z_{1}\right) \pi_{H+\alpha} Y^{\prime}\left(v, z_{2}\right) w=\left(z_{1}-z_{2}\right)^{N} Y^{\prime}\left(v, z_{2}\right) \pi_{H+\alpha} Y^{\prime}\left(u, z_{1}\right) w .
$$

Note that $Y^{\prime}(a, z) \pi_{H}=\pi_{H+\alpha} Y^{\prime}(a, z)$ on $V_{H^{\prime}}$ and $W=V_{H+\alpha} \cdot W$.

By the Claim above, we can introduce a framed VOA structure on

$$
X:=V_{K} \oplus M_{K}\left(\beta, \gamma ;\left.\jmath\right|_{K}\right)
$$

as follows. Since $W$ as a $V_{K}$-module is isomorphic to $M_{K}\left(\beta, \gamma ;\left.\jmath\right|_{K}\right)$, we can identify these structures. For $a, b \in V_{K}$ and $u, v \in M_{K}\left(\beta, \gamma ;\left.\jmath\right|_{K}\right)$, we define the vertex operator map $Y_{X}(\cdot, z)$ by

$$
Y_{X}(a, z) b:=Y^{\prime}(a, z) b, \quad Y_{X}(a, z) u:=Y^{\prime}(a, z) u, \quad Y_{X}(u, z) a:=Y^{\prime}(u, z) a,
$$

and

$$
Y_{X}(u, z) v:=\pi_{H} Y^{\prime}(u, z) v+\pi_{H+\alpha} Y^{\prime}(u, z) v .
$$

Let $\langle\cdot, \cdot\rangle_{U^{\prime}}$ be an non-zero invariant bilinear form on $U^{\prime}$, which is unique up to scalar multiples. Since $V_{K}$ is a subalgebra of $U^{\prime}$, we can define an invariant bilinear form $\langle\cdot, \cdot\rangle_{V_{K}}$ on $V_{K}$ by $\langle a, b\rangle_{V_{K}}:=\langle a, b\rangle_{U^{\prime}}$. In addition, since $W$ as a $V_{K}$-module is isomorphic to $M_{K}\left(\beta, \gamma ;\left.\jmath\right|_{K}\right)$, we may view $\langle\cdot, \cdot\rangle_{U^{\prime}}$ restricted on $W$ as a $V_{K}$-invariant bilinear form on $M_{K}\left(\beta, \gamma ;\left.\jmath\right|_{K}\right)$. Then

$$
\begin{aligned}
\left\langle a, Y_{X}(u, z) v\right\rangle_{V_{K}} & =\left\langle a, \pi_{H} Y^{\prime}(u, z) v\right\rangle_{V_{K}}+\left\langle a, \pi_{H+\alpha} Y^{\prime}(u, z) v\right\rangle_{V_{K}} \\
& =\left\langle a, \pi_{H} Y^{\prime}(u, z) v\right\rangle_{U^{\prime}}+\left\langle a, \pi_{H+\alpha} Y^{\prime}(u, z) v\right\rangle_{U^{\prime}} \\
& =\left\langle a, Y^{\prime}(u, z) v\right\rangle_{U^{\prime}} \\
& =\left\langle Y^{\prime}\left(e^{z L(1)}\left(-z^{-2}\right)^{L(0)} u, z^{-1}\right) a, v\right\rangle_{U^{\prime}} .
\end{aligned}
$$


By the equality above, it follows from Section 5.6 of [FHL] and [Li2] that $Y_{X}(\cdot, z)$ satisfies the Jacobi identity if and only if we have a locality for any three elements in $M_{K}\left(\beta, \gamma ;\left.\jmath\right|_{K}\right)$, which follows from (5.2) and (5.3). Therefore, $\left(X, Y_{X}(\cdot, z)\right)$ is also a framed VOA. In fact, one can define a framed VOA structure on $V_{E} \oplus M_{E}\left(\beta, \gamma ;\left.\jmath\right|_{E}\right)$ for any subcode $E$ of $H^{\prime}$ containing $H$ in a similar way. We shall deduce a contradiction from this observation.

Let $W^{1}$ and $W^{2}$ be $V_{H}$-modules isomorphic to $M_{H}(\beta, \gamma ; \iota)$. Since $W^{i}$ and $M_{K}\left(\beta, \gamma ;\left.\jmath\right|_{K}\right)$ are isomorphic $V_{H}$-modules, we have $V_{H}$-isomorphisms $\varphi_{i}: W^{i} \rightarrow M_{K}\left(\beta, \gamma ;\left.\jmath\right|_{K}\right) \subset X$ for $i=1,2$. Set

$$
X^{\prime}:=V_{H} \oplus V_{H+\alpha} \oplus W^{1} \oplus W^{2} .
$$

We shall define a vertex operator $Y_{X^{\prime}}$ on $X^{\prime}$ as follows.

For $a^{0}, b^{0} \in V_{H}, a^{1}, b^{1} \in V_{H+\alpha}, u^{1}, v^{1} \in W^{1}$ and $u^{2}, v^{2} \in W^{2}$, define

$$
\begin{aligned}
Y_{X^{\prime}}\left(a^{0}, z\right):= & {\left[\begin{array}{cccc}
Y_{X}\left(a^{0}, z\right) & 0 & 0 & 0 \\
0 & Y_{X}\left(a^{0}, z\right) & 0 & 0 \\
0 & 0 & \varphi_{1}^{-1} Y_{X}\left(a^{0}, z\right) \varphi_{1} & 0 \\
0 & 0 & 0 & \varphi_{2}^{-1} Y_{X}\left(a^{0}, z\right) \varphi_{2}
\end{array}\right], } \\
Y_{X^{\prime}}\left(a^{1}, z\right):= & {\left[\begin{array}{cccc}
0 & Y_{X}\left(a^{1}, z\right) & 0 & 0 \\
Y_{X}\left(a^{1}, z\right) & 0 & 0 & 0 \\
0 & 0 & 0 & \varphi_{1}^{-1} Y_{X}\left(a^{1}, z\right) \varphi_{2} \\
0 & 0 & \varphi_{2}^{-1} Y_{X}\left(a^{1}, z\right) \varphi_{1} & 0
\end{array}\right], }
\end{aligned}
$$

$$
\begin{aligned}
& Y_{X^{\prime}}\left(u^{1}, z\right) \\
& :=\left[\begin{array}{cccc}
0 & 0 & \pi_{H} Y_{X}\left(\varphi_{1} u^{1}, z\right) \varphi_{1} & 0 \\
0 & 0 & 0 & \pi_{H+\alpha} Y_{X}\left(\varphi_{1} u^{1}, z\right) \varphi_{2} \\
\varphi_{1}^{-1} Y_{X}\left(\varphi_{1} u^{1}, z\right) & 0 & 0 & 0 \\
0 & \varphi_{2}^{-1} Y_{X}\left(\varphi_{1} u^{1}, z\right) & 0 & 0
\end{array}\right], \\
& Y_{X^{\prime}}\left(u^{2}, z\right) \\
& :=\left[\begin{array}{cccc}
0 & 0 & 0 & \pi_{H} Y_{X}\left(\varphi_{2} u^{2}, z\right) \varphi_{2} \\
0 & 0 & \pi_{H+\alpha} Y_{X}\left(\varphi_{2} u^{2}, z\right) \varphi_{1} & 0 \\
0 & \varphi_{1}^{-1} Y_{X}\left(\varphi_{2} u^{2}, z\right) & 0 & 0
\end{array}\right],
\end{aligned}
$$

on ${ }^{t}\left[b^{0}, b^{1}, v^{1}, v^{2}\right] \in V_{H} \oplus V_{H+\alpha} \oplus W^{1} \oplus W^{2}$. Note that $Y_{X}(\cdot, z)$ is considered as a $V_{H^{-}}$ intertwining operator and $\pi_{H}: X \rightarrow V_{H}$ and $\pi_{\alpha+H}: X \rightarrow V_{\alpha+H}$ are $V_{H}$-projections on $V_{H}$ and $V_{\alpha+H}$, respectively. By (5.2) and (5.3) , it is straightforward to check that $Y_{X^{\prime}}(\cdot, z)$ satisfies the locality and hence $\left(X^{\prime}, Y_{X^{\prime}}(\cdot, z)\right)$ itself forms a VOA. In fact, we have defined a VOA structure on $X^{\prime}$ such that

$$
V^{1} \cdot W^{1}=W^{2}, V^{1} \cdot W^{2}=W^{1}, W^{1} \cdot W^{1}=W^{2} \cdot W^{2}=V^{0} \quad \text { and } \quad W^{1} \cdot W^{2}=V^{2}
$$

based on the framed VOA structure on $X$. 
Now take a subspace

$$
Z:=\left\{a^{0}+b^{0}+u^{1}+\varphi_{2}^{-1} \varphi_{1} u^{1} \in X^{\prime} \mid a^{0} \in V_{H}, b^{0} \in V_{H+\alpha}, u^{1} \in W^{1}\right\} .
$$

Then it follows from the definition of $Y_{X^{\prime}}(\cdot, z)$ that $Z$ is a subalgebra of $X^{\prime}$ and the linear isomorphism

$$
\psi: a^{0}+b^{0}+u^{1}+\varphi_{2}^{-1} \varphi_{1} u^{1} \longmapsto a^{0}+b^{0}+\sqrt{2} \varphi_{1} u^{1}, \quad a^{0} \in V_{H}, b^{0} \in V_{H+\alpha}, \quad u^{1} \in W^{1},
$$

defines a vertex operator algebra isomorphism between $Z$ and $X$. Since $X$ is a framed VOA and every framed VOA is rational, $X^{\prime}$ is a completely reducible $Z$-module. However, since the quotient $X^{\prime} / Z$ has no 1/16-word component corresponding to a codeword 0 , we obtain $\psi^{-1}\left(M_{K}\left(\beta, \gamma ;\left.\jmath\right|_{K}\right)\right) \cdot\left(X^{\prime} / Z\right)=0$ which is a contradiction by Proposition 11.9 of DL]. This contradiction comes from the assumption that $H \neq H^{\prime}$. Hence, $H=H^{\prime}$ as desired.

Now we present the main theorem of this paper:

Theorem 5.5. Let $V=\oplus_{\alpha \in D} V^{\alpha}$ be a framed VOA with structure codes $(C, D)$. Then

(1) For every non-zero $\alpha \in D$, the subcode $C_{\alpha}$ of $C$ contains a doubly even self-dual subcode w.r.t. $\alpha$.

(2) $C$ is even, every codeword of $D$ has a weight divisible by 8, and $D \subset C \subset D^{\perp}$.

Proof: (1) follows from Proposition 5.4 since $V^{0} \oplus V^{\alpha}$ is a framed sub VOA of $V$ for any non-zero $\alpha \in D$. (2) follows from (1), since a self-dual subcode of $C_{\alpha}$ w.r.t. $\alpha$ always contains the codeword $\alpha$.

As a corollary, we can also prove the following theorem.

Theorem 5.6. Let $V=\oplus_{\alpha \in D} V^{\alpha}$ be a framed VOA with structure codes $(C, D)$. Then $V=\oplus_{\alpha \in D} V^{\alpha}$ is a D-graded simple current extension of the code $V O A V^{0}=V_{C}$.

Proof: The assertion follows immediately from Theorem 5.5 and Corollary 4.18 .

There are many applications of Theorems 5.5 and 5.6.

Corollary 5.7. For a positive integer n, the number of isomorphism classes of framed VOAs with a fixed central charge $n / 2$ is finite.

Proof: By Theorem 5.6, every framed VOA is a simple current extension of a code VOA. A code VOA is uniquely determined by its structure code by Proposition 2.3, and it has finitely many irreducible representations as it is rational. In particular, there are finitely many inequivalent simple current modules over a code VOA. Therefore, the number of isomorphism classes of framed VOAs of given central charge is finite by the uniqueness of a simple current extension in Proposition 2.3.

By Theorem 5.6, we can immediately classify all irreducible (both untwisted and $\mathbb{Z}_{2}$ twisted) modules over a framed VOA. 
Corollary 5.8. Let $V=\oplus_{\alpha \in D} V^{\alpha}$ be a framed VOA with structure codes $(C, D)$. Let $W$ be an irreducible $V^{0}$-module. Then there exists $\eta \in \mathbb{Z}_{2}^{n}$, which is unique modulo $D^{\perp}$, such that $W$ can be uniquely extended to an irreducible $\tau_{\eta}$-twisted $V$-module which is given by $V \bigotimes_{V^{0}} W$ as a $V^{0}$-module. In particular, every irreducible untwisted $V$-module is $D$-stable.

Proof: Let $\beta \in C^{\perp}$ be the $1 / 16$-word of $W$. Since all $V^{\alpha}, \alpha \in D$, are simple current $V^{0}$-submodules, the fusion product $W^{\alpha}:=V^{\alpha} \otimes_{V^{0}} W$ is again irreducible. It is clear that the binary $1 / 16$-word of $W^{\alpha}$ is $\alpha+\beta$ so that all $W^{\alpha}, \alpha \in D$, are inequivalent $V^{0}$ modules. Therefore, there exists a unique untwisted or $\mathbb{Z}_{2}$-twisted $V$-module structure on $\operatorname{Ind}_{V^{0}}^{V} W=V \otimes_{V^{0}} W=\oplus_{\alpha \in D} W^{\alpha}$ by Theorem 2.4. Since any element in the dual group $D^{*} \simeq \mathbb{Z}_{2}^{n} / D^{\perp}$ is realized as a Miyamoto involution $\tau_{\eta}$ associated to a codeword $\eta \in \mathbb{Z}_{2}^{n}$, the induced module $\operatorname{Ind}_{V^{0}}^{V} W$ is indeed a $\tau_{\eta}$-twisted $V$-module.

Remark 5.9. By the corollary above and Proposition 2.5, we can compute the fusion rules of $V$-modules from those of $V_{C}$-modules,

Corollary 5.10. ([DGH, $M 3])$ A framed VOA V with structure codes $(C, D)$ is holomorphic if and only if $C=D^{\perp}$.

Proof: That a framed VOA having a structure code $\left(D^{\perp}, D\right)$ is holomorphic is proved in [M3] by showing that every module contains a vacuum-like vector (cf. [Li1). The converse is also proved in [DGH] by using modular forms. Here we give another, rather representation-theoretical proof. Let $V$ be a holomorphic framed VOA with structure codes $(C, D)$ and the $1 / 16$-word decomposition $V=\oplus_{\alpha \in D} V^{\alpha}$. Take any codeword $\delta \in D^{\perp}$. By the previous corollary, a $V_{C}$-module $M_{C}(0, \delta)$ can be uniquely extended to either an untwisted or $\mathbb{Z}_{2}$-twisted $V$-module. As a $V^{0}$-module, it is given by an induced module

$$
V \underset{V_{C}}{\otimes} M_{C}(0, \delta)=\bigoplus_{\alpha \in D} V^{\alpha} \underset{V_{C}}{\otimes} M_{C}(0, \delta) .
$$

By Lemma 4.14, the top weight of $V^{\alpha}$ and that of $V^{\alpha} \bigotimes_{V_{C}} M_{C}(0, \delta)$ are congruent modulo $\mathbb{Z}$ for all $\alpha \in D$. Therefore, the induced module $V \otimes_{V_{C}} M_{C}(0, \delta)$ is an irreducible untwisted $V$-module and thus isomorphic to $V$ itself, as $V$ is holomorphic. Then by considering the 1/16-word decomposition we see that $M_{C}(0, \delta)=V^{0}=M_{C}(0,0)$. Therefore, $\delta \in C$ by Lemma 4.7 and hence $D^{\perp}=C$.

\subsection{Construction of a framed VOA}

In [M3, Y2], certain constructions of a framed VOA are discussed. Assume the following:

(1) $(C, D)$ is a pair of even linear codes of $\mathbb{Z}_{2}^{n}$ such that 
(1-i) $C \subset D^{\perp}$,

(1-ii) for each $\alpha \in D$, there is a subcode $E^{\alpha} \subset C_{\alpha}$ such that

$E^{\alpha}$ is a direct sum of the $[8,4,4]$-Hamming codes.

(2) $V^{0}$ is a code VOA associated to $C$.

(3) $\left\{V^{\alpha} \mid \alpha \in D\right\}$ is a set of irreducible $V^{0}$-modules such that

(3-i) the $1 / 16$-word of $V^{\alpha}$ is $\alpha$,

(3-ii) all $V^{\alpha}, \alpha \in D$, have integral top weights,

(3-iii) the fusion product $V^{\alpha} \bigotimes_{V^{0}} V^{\beta}$ contains $V^{\alpha+\beta}$ for all $\alpha, \beta \in D$.

Then it is shown in [M3, YY2] that the space $V:=\oplus_{\alpha \in D} V^{\alpha}$ forms a framed VOA with structure codes $(C, D)$. Instead of the condition (1-ii), assume that

(1-iii) for each $\alpha \in D, C_{\alpha}$ contains a doubly even self-dual subcode w.r.t. $\alpha$.

Then we have already shown in Lemma 5.1 that $V^{0} \oplus V^{\alpha}$ forms a framed VOA. So by the extension property of simple current extensions in Theorem 2.7, we can again show that $V=\oplus_{\alpha \in D} V^{\alpha}$ forms a framed VOA with structure codes $(C, D)$ under the other conditions. The key idea in [M3, Y2] is to use a special symmetry of the code VOA associated to the $[8,4,4]$-Hamming code to form a minimal $\mathbb{Z}_{2}$-graded extension $V^{0} \oplus V^{\alpha}$. Thanks to Lemma 5.1, we can transcend this step without the [8,4,4]-Hamming code.

Theorem 5.11. With reference to the conditions (1)-(3) above, assume the condition (1-iii) instead of (1-ii). Then $V=\oplus_{\alpha \in D} V^{\alpha}$ forms a framed VOA with structure codes $(C, D)$.

Proof: Let $\left\{\alpha^{i} \mid 1 \leq i \leq r\right\}$ be a linear basis of $D$ and set $D^{(j)}:=\operatorname{Span}_{\mathbb{Z}_{2}}\left\{\alpha_{j} \mid 1 \leq j \leq\right.$ $i\}$. By induction on $i$, we show that the space $V[i]=\oplus_{\alpha \in D^{(i)}} V^{\alpha}$ forms a framed VOA with structure codes $\left(C, D^{(i)}\right)$. The case $i=0$ is trivial, and the case $i=1$ is done in Lemma 5.1. Now assume that $V[i]$ forms a framed VOA for $i \geq 1$. Then we have two simple current extensions $V[i]=\oplus_{\alpha \in D^{(i)}} V^{\alpha}$ and $V^{0} \oplus V^{\alpha^{i+1}}$. Applying Theorem 2.7 to a set $\left\{V^{\alpha} \mid \alpha \in D^{(i+1)}\right\}$ of simple current $V^{0}$-modules, we obtain a $D^{(i+1)}$-graded simple current extension $V[i+1]=\oplus_{\alpha \in D^{(i+1)}} V^{\alpha}$ of $V^{0}$. Repeating this, finally we shall obtain the desired framed VOA structure on $V[r]=\oplus_{\alpha \in D} V^{\alpha}$.

We can also generalize Theorem 7.4 .9 of [Y2] as follows:

Theorem 5.12. Let $V=\oplus_{\alpha \in D} V^{\alpha}$ be a framed $V O A$ with structure codes $(C, D)$. For any even subcode $E$ such that $C \subset E \subset D^{\perp}$, the space

$$
\operatorname{Ind}_{C}^{E} V:=\bigoplus_{\alpha \in D} \operatorname{Ind}_{V_{C}}^{V_{E}} V^{\alpha}=\bigoplus_{\alpha \in D} V_{E} \underset{V_{C}}{\otimes} V^{\alpha}
$$

forms a framed VOA with structure codes $(E, D)$. 
Proof: The idea of the proof is almost the same as that of Theorem 7.4.9 of Y $\mathrm{Y}$ ]. Let $\left\{\gamma^{i}+C \mid 1 \leq i \leq r\right\}$ be a transversal for $C$ in $E$. It is clear that $V_{E}=\oplus_{i=1}^{r} V_{C+\gamma^{i}}$ is an $E / C$-graded simple current extension of $V_{C}$ by Proposition 2.3. First, we show that $V^{\alpha}$ is uniquely extended to an untwisted $V_{E}$-module. For this, it suffices to show that $V_{C+\gamma^{i}} \otimes_{V_{C}} V^{\alpha}, 1 \leq i \leq r$, are inequivalent $V_{C}$-module. Assume $V_{C+\gamma} \otimes_{V_{C}} V^{\alpha} \simeq$ $V_{C+\delta} \bigotimes_{V_{C}} V^{\alpha}$. It follows from a given framed VOA structure and Theorem 5.5 that $V^{\alpha} \bigotimes_{V_{C}} V^{\alpha} \simeq V^{0} \simeq V_{C}$. Since the fusion product is commutative and associative, by multiplying the both sides of $V_{C+\gamma} \bigotimes_{V_{C}} V^{\alpha} \simeq V_{C+\delta} \bigotimes_{V_{C}} V^{\alpha}$ by $V^{\alpha}$ with respect to the fusion product, we have

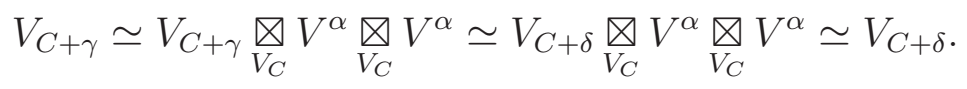

Thus, $\gamma \equiv \delta \bmod C$ and hence all $V_{C+\gamma^{i}} \bigotimes_{V_{C}} V^{\alpha}, 1 \leq i \leq r$, are inequivalent $V_{C}$-modules. Since $E \subset D^{\perp}$, the top weight of $V^{\alpha}$ and that of $V_{C+\gamma^{i}} \bigotimes_{V_{C}} V^{\alpha}$ are congruent modulo $\mathbb{Z}$ by Lemma 4.14. Therefore, $V^{\alpha}$ is uniquely extended to an irreducible untwisted $V_{E}$-module $\operatorname{Ind}_{V_{C}}^{V_{E}} V^{\alpha}=V_{E} \bigotimes_{V_{C}} V^{\alpha}$ by Theorem 2.4. Since all $\operatorname{Ind}_{V_{C}}^{V_{E}} V^{\alpha}, \alpha \in D$, are $E / C$-stable $V_{E}$-modules, we have the fusion rule

$$
\left(\operatorname{Ind}_{V_{C}}^{V_{E}} V^{\alpha}\right) \underset{V_{E}}{\otimes}\left(\operatorname{Ind}_{V_{C}}^{V_{E}} V^{\beta}\right) \simeq \operatorname{Ind}_{V_{C}}^{V_{E}}\left(V^{\alpha} \underset{V_{C}}{\otimes} V^{\beta}\right) \simeq \operatorname{Ind}_{V_{C}}^{V_{E}} V^{\alpha+\beta}
$$

by Proposition 2.5. Therefore, the space

$$
\operatorname{Ind}_{V_{C}}^{V_{E}} V=\bigoplus_{\alpha \in D} \operatorname{Ind}_{V_{C}}^{V_{E}} V^{\alpha}
$$

forms a framed VOA with structure codes $(E, D)$ by Theorem 5.11 ,

By Theorem 5.5, its corollaries and Theorem 5.11, a pair of structure codes $\left(C, C^{\perp}\right)$ of a holomorphic framed VOA satisfies the following conditions.

Condition 1. (F-admissible condition)

(1) The length of $C$ is divisible by 16 .

(2) $C$ is even, every codeword of $C^{\perp}$ has a weight divisible by 8 , and $C^{\perp} \subset C$.

(3) For any $\alpha \in C^{\perp}$, the subcode $C_{\alpha}$ of $C$ contains a doubly even self-dual subcode w.r.t. $\alpha$.

For simplicity, we will call a code $C F$-admissible if it satisfies Condition 1, Indeed, we can construct a holomorphic framed VOA starting from an F-admissible code.

Remark 5.13. A linear code $C$ is F-admissible if and only if its dual $C^{\perp}$ satisfies the following three conditions: 
(i) the length of $C^{\perp}$ is divisible by 16 ,

(ii) $C^{\perp}$ contains the all-one vector,

(iii) $C^{\perp}$ is triply even, that is, $\operatorname{wt}(\alpha)$ is divisible by 8 for any $\alpha \in C^{\perp}$.

For, let $D$ satisfy the conditions (i), (ii) and (iii) above. Then for any $\alpha, \beta \in D$, the weight of their intersection $\alpha \cdot \beta$ is divisible by 4 and so $\alpha \cdot D$ is doubly even. Then there exists a doubly even code $E$ containing $\alpha \cdot D$ such that $E$ is self-dual w.r.t. $\alpha$ by Theorem 5.3. For any $\delta \in(\alpha \cdot D)^{\perp_{\alpha}}$, we have $\langle\delta, D\rangle=\langle\delta \cdot \alpha, D\rangle=\langle\delta, \alpha \cdot D\rangle=0$, showing $E \subset(\alpha \cdot D)^{\perp_{\alpha}} \subset\left(D^{\perp}\right)_{\alpha}$. Therefore, $D^{\perp}$ is F-admissible.

Let $C$ be an $F$-admissible code. Then the all-one vector $1=(11 \ldots 1)$ is contained in $C^{\perp}$. Since $n=\operatorname{wt}(\mathbf{1})$ is divisible by 16 , all irreducible $V_{C}$-modules with the $1 / 16$-word 1 have integral top weights. Let $V^{\mathbf{1}}$ be an irreducible $V_{C}$-modules with the $1 / 16$-word $\mathbf{1}$. Then $V^{1}$ is a self-dual simple current $V_{C^{-}}$module.

Lemma 5.14. Let $C$ be an F-admissible code. For $\alpha \in C^{\perp}, \alpha \notin\{0, \mathbf{1}\}$, there exists an irreducible $V_{C}$-module $W^{\alpha}$ such that $\tau\left(W^{\alpha}\right)=\alpha$ and both $W^{\alpha}$ and the fusion product $V^{1} \otimes_{V_{C}} W^{\alpha}$ have integral top weights.

Proof: Clearly, we can find an irreducible $V_{C}$-module $X$ such that $\tau(X)=\alpha$ and $X$ has an integral top weight. The 1/16-word of the fusion product $V^{1} \otimes_{V_{C}} X$ is then $\mathbf{1}+\alpha$ and its weight is divisible by 8 . Thus, the top weight of $V^{1} \bigotimes_{V_{C}} X$ is in either $\mathbb{Z}$ or $\mathbb{Z}+1 / 2$. In the former case, we just set $W^{\alpha}=X$. If the top weight is in $\mathbb{Z}+1 / 2$, we take a codeword $\delta \in\left(\mathbb{Z}_{2}^{n}\right)_{\alpha}$ such that $\delta$ is odd. By Lemma 4.14, the $V_{C}$-module $\left(V^{1} \bigotimes_{V_{C}} X\right) \bigotimes_{V_{C}} M_{C}(0, \delta)$ has an integral top weight. Set $W^{\alpha}=X \otimes_{V_{C}} M_{C}(0, \delta)$. Since $\operatorname{supp}(\delta) \subset \operatorname{supp}(\alpha)$, the top weight of $X$ is congruent to that of $W^{\alpha}$ modulo $\mathbb{Z}$ by Lemma 4.14, which is integral. Moreover, $V^{1} \otimes_{V_{C}} W^{\alpha} \simeq V^{1} \otimes_{V_{C}}\left(X \otimes_{V_{C}} M_{C}(0, \delta)\right) \simeq\left(V^{1} \otimes_{V_{C}} X\right) \bigotimes_{V_{C}} M_{C}(0, \delta)$ also has an integral top weight as desired.

Proposition 5.15. Let $C$ be an F-admissible code and $D$ a proper subcode of $C^{\perp}$ containing 1. Suppose that we have a framed VOA $V=\oplus_{\alpha \in D} V^{\alpha}$ with structure codes $(C, D)$. Then for $\beta \in C^{\perp} \backslash D$, there exist a self-dual simple current $V$-module $W$ such that $W$ has the 1/16-word decomposition $W=\oplus_{\alpha \in D} W^{\alpha+\beta}$ and $\tilde{V}=V \oplus W$ forms a framed VOA with structure codes $(C, D+\langle\beta\rangle)$.

Proof: By the previous lemma, we can take an irreducible $V_{C^{-}}$-module $W^{\beta}$ such that $\tau\left(W^{\beta}\right)=\beta$ and both $W^{\beta}$ and $V^{\mathbf{1}} \otimes_{V_{C}} W^{\beta}$ are of integral weights. Since $\beta \in C^{\perp}$, it follows from (3) of Condition 1 that $W^{\beta}$ is a self-dual simple current $V_{C^{-}}$-module. Then the induced module $\operatorname{Ind}_{V^{0}}^{V} W^{\beta}=\oplus_{\alpha \in D} V^{\alpha} \nabla_{V_{C}} W^{\beta}$ is an irreducible $\tau_{\eta^{-}}$-twisted $V$-module for some $\eta \in \mathbb{Z}_{2}^{n}$ by Corollary 5.8. If $\eta \in D^{\perp}$, then the space $V \oplus \operatorname{Ind}_{V^{0}}^{V} W^{\beta}$ forms a framed VOA with structure codes $(C, D+\langle\beta\rangle)$ by Theorem 5.11 . 
If $\eta \notin D^{\perp}$, then $\tau_{\eta}$ is not trivial. Set $D^{+}:=\{\alpha \in D \mid\langle\alpha, \eta\rangle=0\}$ and $D^{-}:=\{\alpha \in D \mid$ $\langle\alpha, \eta\rangle=1\}$. Then $D=D^{+} \sqcup D^{-}$and $D^{ \pm} \neq \emptyset$. By our choice of $W^{\beta}$, the all-one vector 1 is in $D^{+}$so that $\eta$ is an even codeword. We set

$$
V^{ \pm}:=\bigoplus_{\alpha \in D^{ \pm}} V^{\alpha}, \quad W^{ \pm}:=\bigoplus_{\alpha \in D^{ \pm}} V^{\alpha} \underset{V_{C}}{\otimes} W^{\beta} .
$$

Then all $V^{ \pm}, W^{ \pm}$are irreducible $V^{+}$-modules. The top weight of $W^{+}$is integral but the top weight of $W^{-}$is in $\mathbb{Z}+1 / 2$. We shall deform $W^{-}$so that it has an integral top weight, also.

Since $\left[\left(D^{+}\right)^{\perp} \cap\langle\beta\rangle^{\perp}, D^{\perp} \cap\langle\beta\rangle^{\perp}\right]=2$, there exists a codeword $\gamma \in\left(D^{+}\right)^{\perp} \cap\langle\beta\rangle^{\perp}$ such that $\left\langle\gamma, D^{-}\right\rangle=1 \bmod 2$. Then it follows from Corollary 5.8 that

$$
\tilde{W}^{ \pm}:=V_{C+\gamma} \underset{V_{C}}{\otimes} W^{ \pm}
$$

are irreducible untwisted $V^{+}$-modules. Moreover, by our choice of $\gamma$, both of $\tilde{W}^{ \pm}$have integral top weights since the top weight of $\tilde{W}^{+}$is congruent to $\langle\gamma, \gamma+\beta\rangle / 2$ modulo $\mathbb{Z}$, whereas the top weight of $\tilde{W}^{-}$is congruent to $\left\langle\gamma, \beta+\gamma+D^{-}\right\rangle / 2+1 / 2$ modulo $\mathbb{Z}$. Therefore, by Theorem 5.11, we have a framed VOA structure on

$$
\tilde{V}:=V^{+} \oplus V^{-} \oplus \tilde{W}^{+} \oplus \tilde{W}^{-}
$$

with structure codes $(C, D+\langle\beta\rangle)$. Now setting $W:=\tilde{W}^{+} \oplus \tilde{W}^{-}$, we have the desired extension of $V$. This completes the proof.

Remark 5.16. In the proof above, we can construct another extension of $V^{+}$which also has the structure codes $(C, D+\langle\beta\rangle)$ in the following way. Take a codeword $\gamma \in D^{\perp}$ with $\langle\gamma, \beta\rangle=1$, which is possible as $\left[D^{\perp}: D^{\perp} \cap\langle\beta\rangle^{\perp}\right]=2$, and set $\tilde{V}^{-}=V_{C+\gamma} \otimes_{V_{C}} V^{-}$and $\tilde{W}^{-}=V_{C+\gamma} \bigotimes_{V_{C}} W^{-}$. Then one can similarly verify that the space $V^{+} \oplus \tilde{V}^{-} \oplus W^{+} \oplus \tilde{W}^{-}$ also forms a framed VOA with structure codes $(C, D+\langle\beta\rangle)$.

Theorem 5.17. There exists a holomorphic framed VOA with structure codes $\left(C, C^{\perp}\right)$ if and only if $C$ is $F$-admissible, i.e., $C$ satisfies Condition 1 .

Proof: Let $\left\{\alpha_{1}, \ldots, \alpha_{r}\right\}$ be a linear basis of $C^{\perp}$ with $\alpha_{1}=1$ and set $D[i]:=\operatorname{Span}_{\mathbb{Z}_{2}}\left\{\alpha_{j} \mid\right.$ $1 \leq j \leq i\}$ for $1 \leq i \leq r$. By Lemma $\left[5.1\right.$ we can construct a framed $V[1]:=V_{C} \oplus V^{1}$ with structure codes $(C, D[1])$. By Proposition [5.15, we can construct a framed VOA $V[2]$ with structure codes $(C, D[2])$ which is a $\mathbb{Z}_{2}$-graded simple current extension of $V[1]$. Recursively, we can construct a $\mathbb{Z}_{2}$-graded simple current extension $V[i+1]$ of $V[i]$ which has structure codes $(C, D[i+1])$ and we shall obtain a holomorphic framed VOA $V[r]$ with structure codes $(C, D[r])=\left(C, C^{\perp}\right)$. 
Remark 5.18. Condition 1, especially (2) and (3), give quite strong restrictions on a code $C$. Roughly speaking, $C$ must be much bigger than its dual $C^{\perp}$ by (3) of Condition 1. In addition, if we assume that the minimum weight of $C$ is greater than 2, then the corresponding framed VOA may have a finite full automorphism group (cf. [LSY], Corollary 3.9]). It seems possible to classify all F-admissible codes $C$ if the length is small. It suggests a possibility for classifying all holomorphic framed VOAs of small central charge. The most interesting (and the first non-trivial) case would be the classification of $c=24$ holomorphic framed VOAs. In fact, one can prove that the moonshine vertex operator algebra $V^{\natural}$ is the unique holomorphic framed VOA of central charge 24 whose weight one subspace is trivial, which is a variant of the famous uniqueness conjecture of the moonshine vertex operator algebra proposed in [FLM] (see also [DGL]). The key point is that the structure codes of $V^{\natural}$ (or any holomorphic framed VOA $V$ of central charge 24 and $\left.V_{1}=0\right)$ are closely related to those of the Leech lattice $V O A V_{\Lambda}$. If $V=\oplus_{\alpha \in C^{\perp}} V^{\alpha}$ with $V^{0} \simeq V_{C}$ is a holomorphic framed VOA of central charge 24 and $V_{1}=0$, then the minimal weight of $C$ is greater than or equal to 4 . In this case, for any $\delta \in \mathbb{Z}_{2}^{48}$ of weight 2 , the $\tau_{\delta}$-twisted orbifold construction yields a VOA

$$
V\left(\tau_{\delta}\right)=\bigoplus_{\alpha \in D}\left(V^{\alpha} \oplus V_{C+\delta} \underset{V_{C}}{\otimes} V^{\alpha}\right), \quad D=\left\{\alpha \in C^{\perp} \mid\langle\alpha, \delta\rangle=0\right\}
$$

which is isomorphic to the Leech lattice $V_{\Lambda}$ and a pair $(C \sqcup(\delta+C), D)$ will be the structure codes of $V_{\Lambda}$. Note that the weight one subspace of $V\left(\tau_{\delta}\right)$ forms an abelian Lie algebra with respect to the bracket $[a, b]=a_{(0)} b$. We shall give more details on this point in our next work [LY].

\section{Frame stabilizers and order four symmetries}

In Section 5, we have seen that structure codes $(C, D)$ of a framed VOA $V=\oplus_{\alpha \in D} V^{\alpha}$ satisfy certain duality conditions. The main property is that for any $\alpha \in D$, the subcode $C_{\alpha}$ contains a doubly even self-dual subcode w.r.t. $\alpha$ and $V^{\alpha}$ is a simple current $V^{0}$ module. However, it is shown in Corollary 4.18 that $V^{\alpha}$ is a simple current module without the assumption on the doubly even property. In this section, we shall discuss the role of the doubly even property. It turns out that by relaxing the doubly even property, we can obtain a refinement of the 1/16-word decomposition and define an automorphism of order four in the pointwise frame stabilizer.

We begin by defining the frame stabilizer and the pointwise frame stabilizer of a framed VOA.

Definition 6.1. Let $V$ be a framed VOA with a frame $F=\operatorname{Vir}\left(e^{1}\right) \otimes \cdots \otimes \operatorname{Vir}\left(e^{n}\right)$. The frame stabilizer of $F$ is the subgroup of all automorphisms of $V$ which stabilizes the 
frame $F$ setwise. The pointwise frame stabilizer is the subgroup of Aut $(V)$ which fixes $F$ pointwise. The frame stabilizer and the pointwise frame stabilizer of $F$ are denoted by $\operatorname{Stab}_{V}(F)$ and $\operatorname{Stab}_{V}^{\mathrm{pt}}(F)$, respectively.

Let $(C, D)$ be the structure code of $V$ with respect to $F$, i.e.,

$$
V=\bigoplus_{\alpha \in D} V^{\alpha}, \quad \tau\left(V^{\alpha}\right)=\alpha \quad \text { and } \quad V^{0}=V_{C}
$$

For any $\theta \in \operatorname{Stab}_{V}^{\mathrm{pt}}(F)$, it is easy to see that $\tau_{e^{i}}=\tau_{\theta e^{i}}=\theta \tau_{e^{i}} \theta^{-1}$ and thus $\theta$ centralizes $\left\langle\tau_{e^{1}}, \ldots, \tau_{e^{n}}\right\rangle$. Therefore, the group $\tau\left(\mathbb{Z}_{2}^{n}\right)=\left\langle\tau_{e^{1}}, \ldots, \tau_{e^{n}}\right\rangle$ generated by the $\tau$-involutions is a central subgroup of $\operatorname{Stab}_{V}^{\mathrm{pt}}(F)$ isomorphic to $\mathbb{Z}_{2}^{n} / D^{\perp}$. In addition, we have $\theta V^{\alpha}=V^{\alpha}$ for all $\alpha \in D$ and hence $\left.\theta\right|_{V^{0}}$ is an automorphism of $V^{0}$.

The following results can be proved easily using the fusion rules.

Lemma 6.2. Let $V=\oplus_{\alpha \in D} V^{\alpha}$ be a framed $V O A$.

(1) Let $\phi \in \operatorname{Aut}\left(V^{0}\right)$ such that $\left.\phi\right|_{F}=\operatorname{id}_{F}$. Then $\phi \in \sigma\left(\mathbb{Z}_{2}^{n}\right)=\left\langle\sigma_{e^{1}}, \ldots, \sigma_{e^{n}}\right\rangle$.

(2) Let $g \in \operatorname{Aut}(V)$ such that $\left.g\right|_{V^{0}}=\mathrm{id}_{V^{0}}$. Then $g \in \tau\left(\mathbb{Z}_{2}^{n}\right)=\left\langle\tau_{e^{1}}, \ldots, \tau_{e^{n}}\right\rangle$.

Proof: (1) Consider the 1/2-word decomposition

$$
V^{0}=\bigoplus_{\beta=\left(\beta_{1}, \ldots, \beta_{n}\right) \in C} L\left(1 / 2, \beta_{1} / 2\right) \otimes \cdots \otimes L\left(1 / 2, \beta_{n} / 2\right)
$$

of $V^{0}$ as an $F=\operatorname{Vir}\left(e^{1}\right) \otimes \cdots \otimes \operatorname{Vir}\left(e^{n}\right)$-module. Since $\left.\phi\right|_{F}=\mathrm{id}_{F}$, it follows from Schur's lemma that $\left.\phi\right|_{V^{0}}$ acts on $L\left(1 / 2, \beta_{1} / 2\right) \otimes \cdots \otimes L\left(1 / 2, \beta_{n} / 2\right)$ by a non-zero scalar $a_{\alpha}$ for each $\alpha \in C$. Moreover, it follows from the fusion rules of $L(1 / 2,0)$-modules in (3.1) that $a_{\alpha} a_{\beta}=a_{\alpha+\beta}$ for all $\alpha, \beta \in C$. Thus the association $C \ni \alpha \mapsto a_{\alpha} \in \mathbb{C}$ defines a character of $C$ and hence there is a codeword $\xi \in \mathbb{Z}_{2}^{n}$ such that $a_{\alpha}=(-1)^{\langle\xi, \alpha\rangle}$. Now it is easy to see that $\left.\phi\right|_{V^{0}}$ is realizable as a product of $\sigma_{e^{i}}, 1 \leq e^{i} \leq n$, that is, $\left.\phi\right|_{V^{0}}=\sigma_{\xi}$.

(2) Since each $V^{\alpha}, \alpha \in D$, is an irreducible $V^{0}$-module, it follows from Schur's lemma that $g$ acts on $V^{\alpha}$ by a non-zero scalar $t_{\alpha} \in \mathbb{C}$. Then again by the fusion rules of $L(1 / 2,0)$ modules in (3.1) we have $t_{\alpha} t_{\beta}=t_{\alpha+\beta}$ so that the map $\alpha \mapsto t_{\alpha}$ defines a character of $D$. Therefore, there exists a codeword $\eta \in \mathbb{Z}_{2}^{n}$ such that $g=\tau_{\eta} \in\left\langle\tau_{e^{1}}, \ldots, \tau_{e^{n}}\right\rangle$.

As a corollary, we have the following theorem.

Theorem 6.3. Let $V$ be a framed VOA with a frame $F=\operatorname{Vir}\left(e^{1}\right) \otimes \cdots \otimes \operatorname{Vir}\left(e^{n}\right)$. For any $\theta \in \operatorname{Stab}_{V}^{\mathrm{pt}}(F)$, there exist $\xi$ and $\eta \in \mathbb{Z}_{2}^{n}$ such that

$$
\left.\theta\right|_{V^{0}}=\sigma_{\xi} \quad \text { and } \quad \theta^{2}=\tau_{\eta} .
$$

In particular, we have $\theta^{4}=1$. 
Let $\theta \in \operatorname{Stab}_{V}^{\mathrm{pt}}(F)$. Then $\left.\theta\right|_{V^{0}}=\sigma_{\xi}$ for some $\xi \in \mathbb{Z}_{2}^{n}$. That means $\theta$ is an extension of a $\sigma$-involution on $V^{0}$ to the whole framed VOA $V$. In this section, we shall give a necessary and sufficient condition on whether a $\sigma$-involution $\sigma_{\xi}$ can be extended to the whole $V$. Our argument is based on the representation theory of code VOAs developed in Section 4 and 5 .

First let us consider $\theta \in \operatorname{Stab}_{V}^{\mathrm{pt}}(F)$ such that $\left.\theta\right|_{V^{0}}=\sigma_{\xi} \neq \mathrm{id}_{V^{0}}$, i.e., $\xi \notin C^{\perp}$. Set $C^{0}:=\{\alpha \in C \mid\langle\xi, \alpha\rangle=0\}$ and $C^{1}:=\{\alpha \in C \mid\langle\xi, \alpha\rangle=1\}$. Then $C^{0}$ is a subcode of $C$, $\left[C: C^{0}\right]=2$ and $C=C^{0} \sqcup C^{1}$. Note also that $V_{C^{0}}$ is fixed by $\theta$ and $\theta$ acts by -1 on $V_{C^{1}}$. In other words, $V^{0}=V_{C^{0}} \oplus V_{C^{1}}$ is the eigenspace decomposition of $\theta$ on $V^{0}$.

Now assume that $\theta^{2}=\tau_{\eta}$ for some $\eta \in \mathbb{Z}_{2}^{n}$. For each non-zero $\alpha \in D$, it is clear that $V^{0} \oplus V^{\alpha}$ is a subalgebra of $V$ and $\theta$ stabilizes $V^{0} \oplus V^{\alpha}$. If $\alpha \in D \cap\langle\eta\rangle^{\perp}$, then $\theta^{2}$ acts as an identity on $V^{0} \oplus V^{\alpha}$ and the eigenvalues of $\theta$ on $V^{\alpha}$ are \pm 1 . Let $V^{\alpha+}$ and $V^{\alpha-}$ be the eigenspaces of $\theta$ with eigenvalues +1 and -1 , respectively. Note that both $V^{\alpha \pm}$ are non-zero inequivalent irreducible $V^{0+}$-submodules. For if $V^{\alpha+}=0$, then $V^{0-} \cdot V^{\alpha-}=V^{\alpha+}=0$, which contradicts Proposition 11.9 of [DL]. Since the subalgebra $V^{0} \oplus V^{\alpha}=V^{0+} \oplus V^{0-} \oplus V^{\alpha+} \oplus V^{\alpha-}$ affords a faithful action of a group $\mathbb{Z}_{2} \times \mathbb{Z}_{2}$ of order 4 , $V^{\alpha \pm}$ are inequivalent irreducible $V^{0+}$-submodules by the quantum Galois theory [DM1].

If $\alpha \in D \backslash\langle\eta\rangle^{\perp}$, then $\theta^{2}=-1$ on $V^{\alpha}$ and the eigenvalues of $\theta$ on $V^{\alpha}$ are $\pm \sqrt{-1}$. Let $V^{\alpha \pm}$ be the eigenspace of $\theta$ of eigenvalues of $\pm \sqrt{-1}$ on $V^{\alpha}$. Then $V^{\alpha}=V^{\alpha+} \oplus V^{\alpha-}$. $V^{\alpha \pm}$ are again non-zero inequivalent irreducible $V^{0+}$-submodules. The argument above actually shows that $\theta$ is of order 2 if and only if $\langle\eta, D\rangle=0$, i.e., $\eta \in D^{\perp}$.

By the observation above, we have

Lemma 6.4. For any $\alpha \in D$, let $V^{\alpha \pm}$ be defined as above. Then the dual $V^{0+}{ }_{-}$module $\left(V^{\alpha \pm}\right)^{*}$ is isomorphic to $V^{\alpha \pm}$ if and only if $\alpha \in\langle\eta\rangle^{\perp}$. Otherwise, $\left(V^{\alpha \pm}\right)^{*}$ is isomorphic to $V^{\alpha \mp}$.

Proof: Since any framed VOA is self-dual, the sub VOA $V^{0} \oplus V^{\alpha}$ of $V$ is also self-dual. Since $V^{\alpha \pm} \cdot V^{\alpha \pm}=V^{0+}$ if and only if $\alpha \in\langle\eta\rangle^{\perp}$, the duality is as in the assertion.

We have shown that for any $\theta \in \operatorname{Stab}_{V}^{\mathrm{pt}}(F) \backslash \tau\left(\mathbb{Z}_{2}^{n}\right),|\theta|=2$ if and only if all irreducible $V^{0+}$-submodules of $V$ are self-dual, and otherwise $|\theta|=4$. We rewrite this condition in terms of the structure codes as follows.

Lemma 6.5. Let $\theta \in \operatorname{Stab}_{V}^{\mathrm{pt}}(F)$ such that $\left.\theta\right|_{V^{0}}=\sigma_{\xi}$ and $\theta^{2}=\tau_{\eta}$ for some $\xi \in \mathbb{Z}_{2}^{n} \backslash C^{\perp}$ and $\eta \in \mathbb{Z}_{2}^{n}$. Set $C^{0}=\{\alpha \in C \mid\langle\xi, \alpha\rangle=0\}$ and $C^{1}=\{\alpha \in C \mid\langle\xi, \alpha\rangle=1\}$.

(1) For $\alpha \in D \cap\langle\eta\rangle^{\perp},\left(C^{0}\right)_{\alpha}$ contains a doubly even self-dual subcode w.r.t. $\alpha$.

(2) For $\alpha \in D \backslash\langle\eta\rangle^{\perp},\left(C^{0}\right)_{\alpha}$ contains a self-dual subcode w.r.t. $\alpha$, but $\left(C^{0}\right)_{\alpha}$ does not contain any doubly even self-dual subcode w.r.t. $\alpha$. 
Proof: (1) For $\alpha \in D \cap\langle\eta\rangle^{\perp}$, let $V^{\alpha}=V^{\alpha+} \oplus V^{\alpha-}$ be the eigenspace decomposition such that $\theta$ acts on $V^{\alpha \pm}$ by \pm 1 . In this case the subspace $V^{0+} \oplus V^{\alpha+}$ forms a framed sub VOA of $V$. By Proposition [5.4, $\left(C^{0}\right)_{\alpha}$ contains a doubly even self-dual subcode w.r.t. $\alpha$.

(2) For $\alpha \in D \backslash\langle\eta\rangle^{\perp}$, let $V^{\alpha}=V^{\alpha+} \oplus V^{\alpha-}$ be the eigenspace decomposition such that $\theta$ acts on $V^{\alpha \pm}$ by $\pm \sqrt{-1}$. In this case the restriction of $\theta$ on the sub VOA

$$
V^{0} \oplus V^{\alpha}=V^{0+} \oplus V^{0-} \oplus V^{\alpha+} \oplus V^{\alpha-}
$$

is of order 4. By the quantum Galois theory [DM1, $V^{\alpha+}$ and $V^{\alpha-}$ are inequivalent irreducible $V^{0+}=V_{C^{0}}$-modules. By Lemma 6.4, $V^{\alpha+}$ and $V^{\alpha-}$ are dual to each other. Therefore, by Proposition 4.11, any maximal self-orthogonal subcode of $\left(C^{0}\right)_{\alpha}$ is not doubly even. Let $H$ be a doubly even self-dual subcode of $C_{\alpha}$ w.r.t. $\alpha$ and $H^{0}$ a maximal self-orthogonal subcode of $\left(C^{0}\right)_{\alpha}$. Since $\left(C^{0}\right)_{\alpha}$ does not contain a doubly even self-dual subcode w.r.t. $\alpha,\left(C^{0}\right)_{\alpha}$ is a proper subgroup of $C_{\alpha}$ so that $\left[C_{\alpha}:\left(C^{0}\right)_{\alpha}\right]=2$. It follows from Theorem 4.3 that $V^{\alpha}$ is a direct sum of $\left[C: C_{\alpha}\right]$ inequivalent $V(0)$-submodules with the multiplicity $\left[C_{\alpha}: H\right]$. Similarly, each of $V^{\alpha \pm}$ is a direct sum of $\left[C^{0}:\left(C^{0}\right)_{\alpha}\right]$ inequivalent irreducible $F$-submodules with the multiplicity $\left[\left(C^{0}\right)_{\alpha}: H^{0}\right]$. Since $V^{\alpha+}$ and $V^{\alpha-}$ are dual to each other, they are isomorphic as F-modules. Therefore, by counting multiplicity of irreducible $F$-submodules of $V^{\alpha}$ and $V^{\alpha \pm}$, one has $\left[C_{\alpha}: H\right]=2\left[\left(C^{0}\right)_{\alpha}: H^{0}\right]$. Combining with $\left|C_{\alpha}\right|=2\left|\left(C^{0}\right)_{\alpha}\right|$, we obtain $|H|=\left|H^{0}\right|=2^{\mathrm{wt}(\alpha) / 2}$. Therefore, $H^{0}$ is a self-dual subcode of $\left(C^{0}\right)_{\alpha}$ w.r.t. $\alpha$.

Lemma 6.6. Let $C$ be an even code and $\beta \in C^{\perp}$. Assume that $V=V_{C} \oplus M_{C}(\beta, \gamma ; \iota)$ forms a framed VOA and $C$ contains a subcode $E$ with index two such that $E$ contains a self-dual subcode w.r.t. $\beta$. Then $V$ decomposes into a direct sum of four inequivalent simple current $V_{E}$-submodules

$$
V=M_{E}(0,0) \oplus M_{E}(0, \delta) \oplus M_{E}(\beta, \gamma ; \jmath) \oplus M_{E}(\beta, \gamma+\delta ; \jmath)
$$

where $\delta$ is an element of $C$ such that $C=E \sqcup(E+\delta)$ and $\jmath: E \cap E^{\perp} \rightarrow \mathbb{C}^{*}$ is a map such that $\left.\jmath\right|_{E \cap C^{\perp}}=\iota$ and $(\alpha, \jmath(\alpha)) \cdot(\beta, \jmath(\beta))=(\alpha+\beta, \jmath(\alpha+\beta)) \in \pi_{\mathbb{C}^{*}}^{-1}(E)$ for all $\alpha, \beta \in E \cap E^{\perp}$. Moreover, all irreducible $V_{E^{-}}$submodules of $V$ are self-dual if and only if $E$ contains a doubly even self-dual subcode w.r.t. $\beta$.

Proof: Let $\delta \in C$ such that $C=E \sqcup(E+\delta)$. Then the decomposition $V_{C}=M_{E}(0,0) \oplus$ $M_{E}(0, \delta)$ is obvious. Let $H$ be a self dual subcode of $E_{\beta}$ w.r.t. $\beta$. Then $H$ is still a maximal self-orthogonal subcode of $C_{\beta}$. Let $\jmath: H \rightarrow \mathbb{C}^{*}$ be an extension of $\iota: C \cap C^{\perp} \rightarrow \mathbb{C}^{*}$ such that $(\alpha, \jmath(\alpha)) \cdot(\beta, \jmath(\beta))=(\alpha+\beta, \jmath(\alpha+\beta))$ for all $\alpha, \beta \in H$. Then $\left.\jmath\right|_{E \cap C^{\perp}}=\iota$ and the decomposition $M_{C}(\beta, \gamma ; \iota)=M_{E}(\beta, \gamma ; \jmath) \oplus M_{E}(\beta, \gamma+\delta ; \jmath)$ follows from Corollary 4.8. That all irreducible $V_{E}$-submodules are simple currents follows from Corollary 4.18, If $E$ 
contains a doubly even self-dual subcode w.r.t. $\beta$, then all irreducible $V_{E \text {-submodules of }}$ $V$ are self-dual by Proposition 4.11. Conversely, if all irreducible $V_{E^{-}}$submodules of $V$ are self-dual, then $V_{E} \oplus M_{E}(\beta, \gamma ; \jmath)$ forms a sub VOA of $V$ so that $E$ contains a doubly even self-dual subcode w.r.t. $\beta$ by Proposition 5.4. This completes the proof.

Theorem 6.7. Let $V$ be a framed $V O A$ with a frame $F=\operatorname{Vir}\left(e^{1}\right) \otimes \cdots \otimes \operatorname{Vir}\left(e^{n}\right)$ and let $(C, D)$ be the corresponding structure codes. For a codeword $\xi \in \mathbb{Z}_{2}^{n} \backslash C^{\perp}$, there exists $\theta \in \operatorname{Stab}_{V}^{\mathrm{pt}}(F)$ such that $\left.\theta\right|_{V^{0}}=\sigma_{\xi}$ if and only if $\alpha \cdot \xi \in C$ for all $\alpha \in D$. Moreover, $|\theta|=2$ if and only if $\operatorname{wt}(\alpha \cdot \xi) \equiv 0 \bmod 4$ for all $\alpha \in D$, and otherwise $|\theta|=4$.

Proof: Let $\theta \in \operatorname{Stab}_{V}^{\mathrm{pt}}(F)$ such that $\left.\theta\right|_{V^{0}}=\sigma_{\xi}$ with $\xi \in \mathbb{Z}_{2}^{n} \backslash C^{\perp}$. Set $C^{0}:=C \cap\langle\xi\rangle^{\perp}$ and $C^{1}:=C \backslash C^{0}$. Then by Lemma 6.5, $\left(C^{0}\right)_{\alpha}$ contains a self-dual subcode w.r.t. $\alpha$ for any $\alpha \in D$. Since

$$
\left(C^{0}\right)_{\alpha}=\{\beta \in C \mid\langle\beta, \xi\rangle=0 \text { and } \operatorname{supp}(\beta) \subset \operatorname{supp}(\alpha)\}
$$

and $\langle\beta, \xi\rangle=\langle\beta, \alpha \cdot \xi\rangle=0$ for all $\beta \in\left(C^{0}\right)_{\alpha}, \alpha \cdot \xi \in\left(\left(C^{0}\right)_{\alpha}\right)^{\perp}$ for all $\alpha \in D$. Therefore, $\alpha \cdot \xi$ is contained in all self-dual subcodes of $\left(C^{0}\right)_{\alpha}$ w.r.t. $\alpha$ and hence $\alpha \cdot \xi \in\left(C^{0}\right)_{\alpha} \subset C$ as claimed.

Conversely, assume that a codeword $\xi \in \mathbb{Z}_{2}^{n} \backslash C^{\perp}$ satisfies $\alpha \cdot \xi \in C$ for all $\alpha \in D$. Then $C^{0}=C \cap\langle\xi\rangle^{\perp}$ is a proper subcode of $C$ with index 2. By definition, $\alpha \cdot \xi \in\left(\left(C^{0}\right)_{\alpha}\right)^{\perp}$ for all $\alpha \in D$. Therefore, any maximal self-orthogonal subcode of $\left(C^{0}\right)_{\alpha}$ contains $\alpha \cdot \xi$. Set $D^{0}:=\{\alpha \in D \mid \operatorname{wt}(\alpha \cdot \xi) \equiv 0 \bmod 4\}$ and $D^{1}:=\{\alpha \in D \mid \operatorname{wt}(\alpha \cdot \xi) \equiv 2 \bmod 4\}$. It is clear that $D=D^{0} \sqcup D^{1}$. If $\alpha \in D^{0}$, then there exists a doubly even self-dual subcode of $\left(C^{0}\right)_{\alpha}$ w.r.t. $\alpha$. For, let $H$ be a doubly even self-dual subcode of $C_{\alpha}$, which exists by Proposition 5.4. If $H$ is contained in $\left(C^{0}\right)_{\alpha}$, then we are done. If not, then $\alpha \cdot \xi \notin H$ and $H \cap\left(C^{0}\right)_{\alpha}=H \cap\langle\xi\rangle^{\perp}$ is a subcode of $H$ with index 2 so that

$$
\left(H \cap\langle\xi\rangle^{\perp}\right) \sqcup\left(H \cap\langle\xi\rangle^{\perp}+\alpha \cdot \xi\right)
$$

gives a doubly even self-dual subcode of $\left(C^{0}\right)_{\alpha}$ w.r.t. $\alpha$. Similarly, we can show that $\left(C^{0}\right)_{\alpha}$ contains a self-dual subcode w.r.t. $\alpha$ for any $\alpha \in D^{1}$. But in this case any self-dual subcode of $\left(C^{0}\right)_{\alpha}$ w.r.t. $\alpha$ is not doubly even, as it always contains $\alpha \cdot \xi$. We have shown that for each $\alpha \in D,\left(C^{0}\right)_{\alpha}$ contains a self-dual subcode w.r.t. $\alpha$ so that one has a $V_{C^{0} \text {-module }}$ decomposition $V^{\alpha}=V^{\alpha, 1} \oplus V^{\alpha, 2}$ and $V^{\alpha, p}, p=1,2$, are simple current $V_{C^{0} \text {-submodules }}$ by Lemma 6.6.

Let $\left\{\alpha^{1}, \ldots, \alpha^{r}\right\}$ be a linear basis of $D^{0}$. For each $i, 1 \leq i \leq r$, choose an irreducible $V_{C^{0}}$-submodule $U^{\alpha^{i}}$ of $V^{\alpha^{i}}$ arbitrary. Then for $\alpha=\alpha^{i_{1}}+\cdots+\alpha^{i_{k}} \in D^{0}$, set

$$
U^{\alpha}:=U^{\alpha^{i_{1}}} \underset{V_{C^{0}}}{\bigotimes} \cdots \underset{V_{C^{0}}}{\bigotimes} U^{\alpha^{i} k}
$$




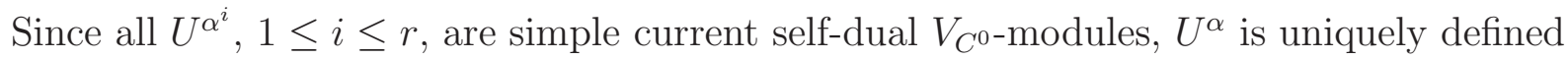
by (6.1) for all $\alpha \in D^{0}$. Note that $U^{0}=V_{C^{0}}$. Since $\oplus_{\alpha \in D^{0}} V^{\alpha}$ is a sub VOA of $V, U^{\alpha}$

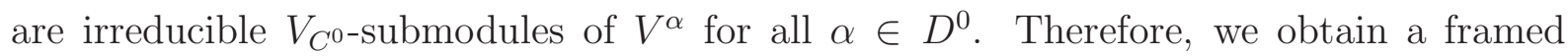
sub VOA $U:=\oplus_{\alpha \in D^{0}} U^{\alpha}$ of $V$ with structure codes $\left(C^{0}, D^{0}\right)$. It is easy to see that $V^{\alpha}=U^{\alpha} \oplus\left(V_{C^{1}} \otimes_{V_{C^{0}}} U^{\alpha}\right)$ for $\alpha \in D^{0}$ by Lemma 6.6.

If $D=D^{0}$, then we have $V=U \oplus\left(V_{C^{1}} \bigotimes_{V_{C^{0}}} U\right)$ as a $V_{C^{0}}$-module. In this case we define a linear automorphism $\theta_{\xi}$ on $V$ by

$$
\theta_{\xi}:=\left\{\begin{aligned}
1 & \text { on } U \\
-1 & \text { on } V_{C^{1}} \otimes_{V_{C^{0}}} U
\end{aligned}\right.
$$

Then it follows from Lemma 6.6 and Proposition 4.15 that $\theta_{\xi} \in \operatorname{Stab}_{V}^{\mathrm{pt}}(F)$ and $\left.\theta_{\xi}\right|_{V^{0}}=\sigma_{\xi}$. Therefore, $\sigma_{\xi}$ can be extended to an involution on $V$.

If $D \neq D^{0}$, then $D=D^{0} \sqcup D^{1}$ with $D^{1} \neq \emptyset$. In this case, take one $\beta \in D^{1}$ and an

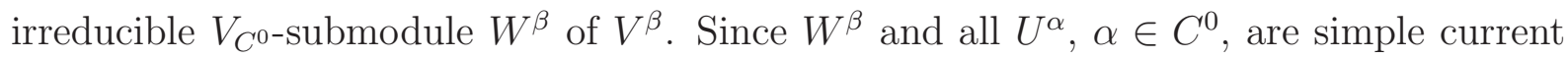
$V_{C^{0}}$-modules, we have a $V_{C^{0}}$-module decomposition

$$
V^{\alpha+\beta}=\left(U^{\alpha} \underset{V_{C^{0}}}{\bigotimes} W^{\beta}\right) \oplus\left(V_{C^{1}} \underset{V_{C^{0}}}{\bigotimes} U^{\alpha} \underset{V_{C^{0}}}{\bigotimes} W^{\beta}\right)
$$

of $V^{\alpha+\beta}$ for all $\alpha \in C^{0}$ by Lemma 6.6. Since $\left(C^{0}\right)_{\alpha+\beta}$ contains no doubly even self-dual subcode w.r.t. $\alpha+\beta$, the decomposition

$$
V^{0} \oplus V^{\alpha+\beta}=V_{C^{0}} \oplus V_{C^{1}} \oplus\left(U^{\alpha} \underset{V_{C^{0}}}{\otimes} W^{\beta}\right) \oplus\left(V_{C^{1}} \underset{V_{C^{0}}}{\otimes} U^{\alpha} \underset{V_{C^{0}}}{\otimes} W^{\beta}\right)
$$

induces an order four automorphism on a sub VOA $V^{0} \oplus V^{\alpha+\beta}$ of $V$ by Lemma 6.6 and Proposition 4.15, Set

$$
W:=\bigoplus_{\alpha \in C^{0}} U^{\alpha} \underset{V_{C^{0}}}{\otimes} W^{\beta}
$$

Then we have obtained the following decomposition of $V$ as a $V_{C^{0}}$-module:

$$
V=U \oplus\left(V_{C^{1}} \underset{V_{C^{0}}}{\otimes} U\right) \oplus W \oplus\left(V_{C^{1}} \underset{V_{C^{0}}}{\bigotimes} W\right)
$$

We define a linear automorphism $\theta_{\xi}$ on $V$ by

$$
\theta_{\xi}:= \begin{cases}1 & \text { on } U \\ -1 & \text { on } V_{C^{1}} \bigotimes_{V_{C^{0}}} U \\ \sqrt{-1} & \text { on } W \\ -\sqrt{-1} & \text { on } V_{C^{1}} \bigotimes_{V_{C^{0}}} W .\end{cases}
$$

Then it follows from the argument above that $\theta_{\xi} \in \operatorname{Stab}_{V}^{\mathrm{pt}}(F)$ and $\left.\theta_{\xi}\right|_{V^{0}}=\sigma_{\xi}$. Therefore, $\sigma_{\xi}$ gives rise to an automorphism of order 4 . 
Summarizing, we have shown that there exists $\theta \in \operatorname{Stab}_{V}^{\mathrm{pt}}(F)$ such that $\left.\theta\right|_{V^{0}}=\sigma_{\xi}$ if and only if $\alpha \cdot \xi \in C$ for all $\alpha \in D$. It remains to show that for such $\theta,|\theta|=2$ if and only if $\operatorname{wt}(\alpha \cdot \xi) \equiv 0 \bmod 4$. But this is almost obvious by the preceding argument.

Remark 6.8. Let $V=\oplus_{\alpha \in D} V^{\alpha}$ be a framed VOA with structure codes $(C, D)$. It was conjectured in [M3, Conjecture 1] that for any codeword $\beta \in C$, the $\sigma$-type involution $\sigma_{\beta} \in$ $\operatorname{Aut}\left(V^{0}\right)$ can be extended to an automorphism of $V$, that means there exists $g \in \operatorname{Stab}_{V}^{\mathrm{pt}}(F)$ such that $\left.g\right|_{V^{0}}=\sigma_{\beta}$. By the theorem above, we know that this is not correct; we have to take a codeword $\beta$ such that $\alpha \cdot \beta \in C$ for all $\alpha \in D$.

Motivated by Theorem 6.7, we define $P:=\left\{\xi \in \mathbb{Z}_{2}^{n} \mid \alpha \cdot \xi \in C\right.$ for all $\left.\alpha \in D\right\}$. It is clear that $P$ is a linear subcode of $C$. Moreover, we have

Lemma 6.9. $C^{\perp} \subset P$.

Proof: Let $\delta \in C^{\perp}$. For $\alpha \in D$, one has $\left\langle\delta, C_{\alpha}\right\rangle=0$ by definition and hence $\left\langle\alpha \cdot \delta, C_{\alpha}\right\rangle=$ 0. Since $C_{\alpha}$ contains a self-dual subcode w.r.t. $\alpha$ by Theorem 5.5, $\alpha \cdot \delta \in C_{\alpha} \subset C$.

For each codeword $\xi \in P$, there exists $\theta_{\xi} \in \operatorname{Stab}_{V}^{\mathrm{pt}}(F)$ such that $\left.\theta_{\xi}\right|_{V^{0}}=\sigma_{\xi}$ by Theorem 6.7. However, the construction of $\theta_{\xi}$ in the proof of Theorem 6.7 is not unique since we

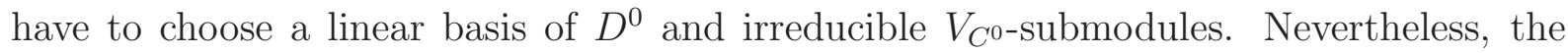
following holds.

Lemma 6.10. Let $\theta, \phi \in \operatorname{Stab}_{V}^{\mathrm{pt}}(F)$ such that $\left.\theta\right|_{V^{0}}=\left.\phi\right|_{V^{0}}=\sigma_{\xi}$. Then $\phi=\theta \tau_{\eta}$ for some $\eta \in \mathbb{Z}_{2}^{n}$.

Proof: Since $\left.\theta\right|_{V^{0}}=\left.\phi\right|_{V^{0}}$, we have $\left.\theta^{-1} \phi\right|_{V^{0}}=\mathrm{id}_{V^{0}}$. By Lemma 6.2, there exists $\eta \in \mathbb{Z}_{2}^{n}$ such that $\theta^{-1} \phi=\tau_{\eta}$ and hence $\phi=\theta \tau_{\eta}$ as desired.

In other words, $\theta_{\xi}$ is only determined modulo $\tau$-involutions. We have also seen in Lemma 6.2 that $\theta_{\xi} \in \tau\left(\mathbb{Z}_{2}^{n}\right)$ if and only if $\xi \in C^{\perp}$. Since $C^{\perp} \subset P$ by Lemma 6.9, the association $\xi+C^{\perp} \mapsto \theta_{\xi} \tau\left(\mathbb{Z}_{2}^{n}\right)$ defines a group isomorphism between $P / C^{\perp}$ and $\operatorname{Stab}_{V}^{\mathrm{pt}}(F) / \tau\left(\mathbb{Z}_{2}^{n}\right)$. Therefore, we have the following central extension:

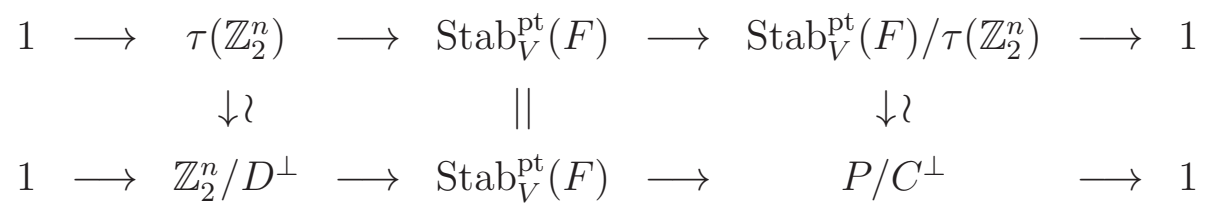

The commutator relation in $\operatorname{Stab}_{V}^{\mathrm{pt}}(F)$ can also be described as follows.

Theorem 6.11. For $\xi^{1}, \xi^{2} \in P$, let $\theta_{\xi^{i}}, i=1,2$, be extensions of $\sigma_{\xi^{i}}$ to $\operatorname{Stab}_{V}^{\mathrm{pt}}(F)$. Then $\left[\theta_{\xi^{1}}, \theta_{\xi^{2}}\right]=1$ if and only if $\left\langle\alpha \cdot \xi^{1}, \alpha \cdot \xi^{2}\right\rangle=0$ for all $\alpha \in D$. 
Proof: Since the case $\theta_{\xi^{1}} \in \theta_{\xi^{2}} \tau\left(\mathbb{Z}_{2}^{n}\right)$ is trivial, we assume that $\sigma_{\xi^{1}} \neq \sigma_{\xi^{2}}$. For $i=1,2$, set $C^{0, \xi^{i}}:=\left\{\alpha \in C \mid\left\langle\alpha, \xi^{i}\right\rangle=0\right\}$ and $E:=C^{0, \xi^{1}} \cap C^{0, \xi^{2}}$. Then $C^{0, \xi^{i}}$ are subcodes of $C$ with index 2 and $E$ is a subcode of $C$ with index 4. Let $\delta^{1}, \delta^{2} \in C$ such that $C^{0, \xi^{i}}=E \sqcup\left(E+\delta^{i}\right)$. By definition, $\alpha \cdot \xi^{1}, \alpha \cdot \xi^{2} \in\left(E_{\alpha}\right)^{\perp}$ for all $\alpha \in D$ so that $E_{\alpha}$ contains a self-dual subcode w.r.t. $\alpha$ if and only if $\left\langle\alpha \cdot \xi^{1}, \alpha \cdot \xi^{2}\right\rangle=0$. We have seen that $\theta_{\xi^{i}}$ acts semisimply on each $V^{\alpha}, \alpha \in D$, with two eigenvalues, and these eigenspaces

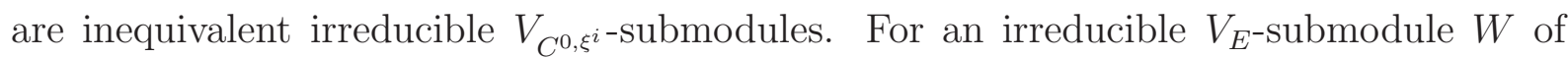
$V^{\alpha}$, the subspace $W+\left(V_{E+\delta^{i}} \cdot W\right)$ forms a $V_{C^{0, \xi^{i}}}$-submodule so that $\theta_{\xi^{i}}$ acts on $W$ by an eigenvalue. Therefore, $\theta_{\xi^{1}}$ commutes with $\theta_{\xi^{2}}$ if and only if $V^{\alpha}$ splits into a direct sum of 4 irreducible $V_{E}$-submodules for all $\alpha \in D$. Let $m_{\alpha}$ be the number of irreducible

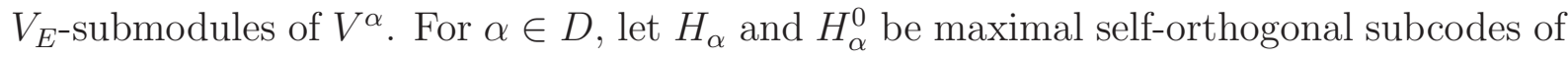
$C_{\alpha}$ and $E_{\alpha}$, respectively. By the structure of irreducible modules over a code VOA shown in Theorem [4.3, $V^{\alpha}$ is a direct sum of $[C: H]$ irreducible $F$-submodules. Moreover,

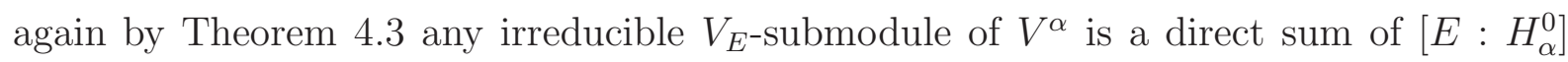
irreducible $F$-submodules. By counting the number of irreducible $F$-submodules of $V^{\alpha}$, we have $m_{\alpha}\left|H_{\alpha}\right|=4\left|H_{\alpha}^{0}\right|$ as $[C: E]=4$. Thus, $E_{\alpha}$ contains a self-dual subcodes w.r.t. $\alpha$ if and only if $m_{\alpha}=4$. Hence, $\theta_{\xi^{1}}$ commutes with $\theta_{\xi^{2}}$ if and only if $\left\langle\alpha \cdot \xi^{1}, \alpha \cdot \xi^{2}\right\rangle=0$.

We have shown that the structure of $\operatorname{Stab}_{V}^{\mathrm{pt}}(F)$ is determined by Theorems 6.7 and 6.11 only in terms of the structure codes $(C, D)$.

Remark 6.12. In [Y2, $[Y 3]$, one of the authors has shown that for any Ising vector $e \in V^{\natural}$, we have no automorphism $g \in \operatorname{Aut}\left(V^{\natural}\right)$ such that $g$ restricted on $\left(V^{\natural}\right)^{\left\langle\tau_{e}\right\rangle}$ is equal to $\sigma_{e}$. Thanks to Theorem [6.7, we can give a simpler proof of this. As shown in [DMZ], the moonshine VOA $V^{\natural}$ is framed. Take any Ising frame $F=\operatorname{Vir}\left(e^{1}\right) \otimes \cdots \otimes \operatorname{Vir}\left(e^{48}\right)$ of $V^{\natural}$ and set $\xi=\left(10^{47}\right) \in \mathbb{Z}_{2}^{48}$. Since $\xi$ is odd, there is no extension of $\sigma_{\xi}=\sigma_{e^{1}}$ to $\operatorname{Stab}_{V^{\natural}}^{\mathrm{pt}}(F)$ by Theorem 6.7. Since all the Ising vectors of $V^{\natural}$ are conjugate under $\operatorname{Aut}\left(V^{\natural}\right)=\mathbb{M}(c f$. $[\mathrm{C}, \mathrm{M1}])$, e and $e^{1}$ are conjugate. Therefore, there is no extension of $\sigma_{e}$ to $V^{\natural}$.

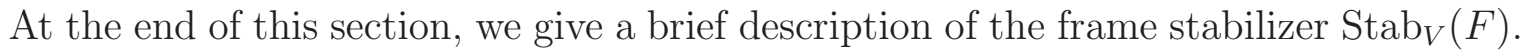
Its structure is also discussed in [DGH]. It is clear that $\operatorname{Stab}_{V}^{\mathrm{pt}}(F)$ is a normal subgroup of $\operatorname{Stab}_{V}(F)$. Let $g \in \operatorname{Stab}_{V}(F)$. Then $g$ induces a permutation $\mu_{g} \in \mathrm{S}_{n}$ on the set of Ising vectors $\left\{e^{1}, \ldots, e^{n}\right\}$ of $F$, namely $g e^{i}=e^{\mu_{g}(i)}$. Since $g$ preserves the $1 / 16$-word decomposition $V=\oplus_{\alpha \in D} V^{\alpha}$, it follows that $g V^{\alpha}=V^{\mu_{g}(\alpha)}$ with $\mu_{g}(\alpha)=\left(\alpha_{\mu_{g}(1)}, \ldots, \alpha_{\mu_{g}(n)}\right)$. In particular, $g$ restricted on $V^{0}$ defines an element of $\operatorname{Aut}\left(V^{0}\right)=\operatorname{Aut}\left(V_{C}\right)$ which is a lift of $\operatorname{Aut}(C)$. Therefore, every element of $\operatorname{Stab}_{V}(F)$ is a lift of $\operatorname{Aut}(C) \cap \operatorname{Aut}(D)$. Conversely, we know that for any $\mu \in \operatorname{Aut}(C)$, there exists $\tilde{\mu} \in \operatorname{Aut}\left(V_{C}\right)$ such that $\tilde{\mu} e^{i}=e^{\mu(i)}$ for $1 \leq i \leq n$ by Theorem 3.3 of [Sh. It is shown in Lemma 3.15 of [SY] that if $\tilde{\mu}$ lifts to an element of $\operatorname{Aut}(V)$ then $\left\{\left(V^{\alpha}\right)^{\tilde{\mu}} \mid \alpha \in D\right\}$ coincides with $\left\{V^{\alpha} \mid \alpha \in D\right\}$ as a set of inequivalent irreducible $V_{C^{-}}$-modules. Therefore, there exists a lift of $\tilde{\mu} \in \operatorname{Aut}\left(V_{C}\right)=\operatorname{Aut}\left(V^{0}\right)$ to 
an element of $\operatorname{Aut}(V)$ if and only if the subgroup $\left\{V^{\alpha} \mid \alpha \in D\right\}$ of the group formed by all the simple current $V_{C}$-module in the fusion algebra is invariant under the conjugation action of $\tilde{\mu}$. If such a lift of $\tilde{\mu}$ exists, it is unique modulo $\operatorname{Stab}_{V}^{\mathrm{pt}}(F)$. For if $\tilde{\mu}$ and $\tilde{\mu}^{\prime}$ are two lifts of $\mu, \tilde{\mu}^{-1} \tilde{\mu}^{\prime}$ fixes $F$ pointwise, showing $\tilde{\mu}^{-1} \tilde{\mu}^{\prime} \in \operatorname{Stab}_{V}^{\mathrm{pt}}(F)$. Thus, the factor group $\operatorname{Stab}_{V}(F) / \operatorname{Stab}_{V}^{\mathrm{pt}}(F)$ is isomorphic to a subgroup of $\operatorname{Aut}(C) \cap \operatorname{Aut}(D)$ which gives a slight refinement of (3) of Theorem 2.8 in [DGH]. The $V_{C^{-}}$-module structure of $\left(V^{\alpha}\right)^{\tilde{\mu}}$ involves some extra information other than $C$ and $D$, namely, if $V^{\alpha} \simeq M_{C}\left(\alpha, \gamma ; \iota_{\alpha}\right)$ for $\alpha \in D$ then $\left(V^{\alpha}\right)^{\tilde{\mu}} \simeq M_{C}\left(\mu^{-1} \alpha, \gamma^{\prime} ; \iota_{\mu^{-1} \alpha}\right)$ for some codeword $\gamma^{\prime} \in \mathbb{Z}_{2}^{n}$, and this $\gamma^{\prime}$ depends not only on $C$ and $D$ but also on $\gamma, \iota_{\alpha}$ and $\iota_{\mu^{-1} \alpha}$. We do not have a general result for the lifting property of $\operatorname{Aut}(C) \cap \operatorname{Aut}(D)$ at present.

\section{4A-twisted orbifold construction}

Let $V^{\natural}$ be the moonshine VOA constructed in [FLM]. In this section, we shall apply Theorem 6.7 to define a $4 \mathrm{~A}$-element of the Monster $\mathbb{M}=\operatorname{Aut}\left(V^{\natural}\right)$ and exhibit that the 4A-twisted orbifold construction of the moonshine VOA $V^{\natural}$ will be $V^{\natural}$ itself.

By [DGH, M3, we can take an Ising frame $F=\operatorname{Vir}\left(e^{1}\right) \otimes \cdots \otimes \operatorname{Vir}\left(e^{48}\right)$ of $V^{\natural}$ such that the associated structure codes $(\mathcal{C}, \mathcal{D})$ are as follows:

$$
\mathcal{C}=\mathcal{D}^{\perp}, \quad \mathcal{D}=\operatorname{Span}_{\mathbb{Z}_{2}}\left\{\left(1^{16} 0^{32}\right),\left(0^{32} 1^{16}\right),(\alpha, \alpha, \alpha) \mid \alpha \in \operatorname{RM}(1,4)\right\},
$$

where $\operatorname{RM}(1,4) \subset \mathbb{Z}_{2}^{16}$ is the first order Reed-Muller code defined by the generator matrix

$\left[\begin{array}{llll}1111 & 1111 & 1111 & 1111 \\ 1111 & 1111 & 0000 & 0000 \\ 1111 & 0000 & 1111 & 0000 \\ 1100 & 1100 & 1100 & 1100 \\ 1010 & 1010 & 1010 & 1010\end{array}\right]$

Note that

$$
\mathcal{C}=\left\{(\alpha, \beta, \gamma) \in \mathbb{Z}_{2}^{48} \mid \alpha, \beta, \gamma \in \mathbb{Z}_{2}^{16} \text { are even and } \alpha+\beta+\gamma \in \mathrm{RM}(2,4)\right\}
$$

Remark 7.1. The weight enumerator of $\operatorname{RM}(1,4)$ is $X^{16}+30 X^{8} Y^{8}+Y^{16}$.

Let $V^{\natural}=\oplus_{\alpha \in \mathcal{D}}\left(V^{\natural}\right)^{\alpha}$ be the 1/16-word decomposition. Set

$$
\mathcal{P}:=\left\{\xi \in \mathbb{Z}_{2}^{48} \mid \alpha \cdot \xi \in \mathcal{C} \text { for all } \alpha \in \mathcal{D}\right\}
$$

Then for each $\xi \in \mathcal{P}$, one can define an automorphism $\theta_{\xi} \in \operatorname{Stab}_{V^{\natural}}^{\mathrm{pt}}(F)$ such that $\left.\theta_{\xi}\right|_{V^{0}}=$ $\sigma_{\xi}$ by Theorem 6.7. Note also that $\mathcal{D}=\mathcal{C}^{\perp}<\mathcal{P}<\mathcal{C}$ and $\sigma_{\xi^{1}}=\sigma_{\xi^{2}}$ if and only if $\xi^{1}+\xi^{2} \in \mathcal{C}^{\perp}=\mathcal{D}$. 
Lemma 7.2. Let $\mathcal{C}, \mathcal{D}$ and $\mathcal{P}$ be defined as above. Then

$$
\mathcal{P}=\left\{(\alpha, \beta, \gamma) \in \mathbb{Z}_{2}^{48} \mid \alpha, \beta, \gamma \in \operatorname{RM}(2,4) \text { and } \alpha+\beta+\gamma \in \operatorname{RM}(1,4)\right\},
$$

where $\operatorname{RM}(2,4)=\operatorname{RM}(1,4)^{\perp} \subset \mathbb{Z}_{2}^{16}$ is the second order Reed-Muller code of length 16 .

Proof: Set $E:=\{(\alpha, \beta, \gamma) \mid \alpha, \beta, \gamma \in \operatorname{RM}(2,4)$ and $\alpha+\beta+\gamma \in \operatorname{RM}(1,4)\}$. We shall first show that $E \subset \mathcal{P}$. It is clear that if $d_{1} \cdot \xi, d_{2} \cdot \xi \in \mathcal{C}$ then $\left(d_{1}+d_{2}\right) \cdot \xi=d_{1} \cdot \xi+d_{2} \cdot \xi \in \mathcal{C}$. Thus, we only need to show that $d \cdot \xi \in \mathcal{C}$ for $\xi \in E$ and $d$ in a generating set of $\mathcal{D}$.

Let $\xi=\left(\xi^{1}, \xi^{2}, \xi^{3}\right) \in E$ with each $\xi^{i} \in \mathbb{Z}_{2}^{16}$. Then $\xi^{1}, \xi^{2}, \xi^{3} \in \operatorname{RM}(2,4)$. Hence, $\alpha \cdot \xi \in \mathcal{C}$ for $\alpha=\left(1^{16} 0^{32}\right),\left(0^{16} 1^{16} 0^{16}\right)$ and $\left(0^{32} 1^{16}\right) \in \mathcal{D}$. Take any two codewords $\beta, \gamma \in \operatorname{RM}(1,4)$ with weight 8 . Then the weight of $\beta \cdot \gamma$ is either 0,4 or 8 by Remark 7.1. By the definition of $E$, we have $\xi^{1}+\xi^{2}+\xi^{3} \in \operatorname{RM}(1,4)$. Therefore,

$$
\left\langle\beta, \gamma \cdot\left(\xi^{1}+\xi^{2}+\xi^{3}\right)\right\rangle \equiv \operatorname{wt}\left(\beta \cdot \gamma \cdot\left(\xi^{1}+\xi^{2}+\xi^{3}\right)\right) \equiv 0 \bmod 2
$$

and hence $\gamma \cdot\left(\xi^{1}+\xi^{2}+\xi^{3}\right) \in \operatorname{RM}(1,4)^{\perp}=\operatorname{RM}(2,4)$. Since $\xi^{i} \in \operatorname{RM}(2,4)=\operatorname{RM}(1,4)^{\perp}$, all $\gamma \cdot \xi^{i}, i=1,2,3$, are even codewords. Therefore, $(\gamma, \gamma, \gamma) \cdot \xi=\left(\gamma \cdot \xi^{1}, \gamma \cdot \xi^{2}, \gamma \cdot \xi^{3}\right) \in \mathcal{C}$ for all $\gamma \in \operatorname{RM}(1,4)$. As $\mathcal{D}$ is generated by the elements of the form: $\left(1^{16} 0^{32}\right),\left(0^{16} 1^{16} 0^{16}\right),\left(0^{32} 1^{16}\right)$ and $(\gamma, \gamma, \gamma)$ with $\gamma \in \operatorname{RM}(1,4)$, we have $E \subset \mathcal{P}$.

Conversely, assume $\left(\alpha^{1}, \alpha^{2}, \alpha^{3}\right) \in \mathcal{P}$ with $\alpha^{i} \in \mathbb{Z}_{2}^{16}$. Then one has $\left(\alpha^{1}, \alpha^{2}, \alpha^{3}\right) \cdot \beta \in \mathcal{C}$ for $\beta=\left(1^{16} 0^{32}\right),\left(0^{16} 1^{16} 0^{16}\right)$ and $\left(0^{32} 1^{16}\right) \in \mathcal{D}$ so that $\alpha^{i} \in \operatorname{RM}(2,4)$ for $i=1,2,3$. Moreover, for $(\gamma, \gamma, \gamma) \in \mathcal{D}$ with $\gamma \in \operatorname{RM}(1,4),\left(\alpha^{1}, \alpha^{2}, \alpha^{3}\right) \cdot(\gamma, \gamma, \gamma) \in \mathcal{C}$ is an even codeword. Then it follows from (7.1) that $\left(\alpha^{1}+\alpha^{2}+\alpha^{3}\right) \cdot \gamma \in \operatorname{RM}(2,4)$ and thus $\alpha^{1}+\alpha^{2}+\alpha^{3} \in \operatorname{RM}(1,4)$. Hence $E=\mathcal{P}$.

Take

$$
\xi=(110000001100000001100000011000001010000010100000) \in \mathbb{Z}_{2}^{48} .
$$

Then $\xi \in \mathcal{P}$ by Lemma 7.2. Set $\mathcal{D}^{0}:=\{\alpha \in \mathcal{D} \mid \operatorname{wt}(\alpha \cdot \xi) \equiv 0 \bmod 4\}$ and $\mathcal{D}^{1}:=\{\alpha \in$ $\mathcal{D} \mid \operatorname{wt}(\alpha \cdot \xi) \equiv 2 \bmod 2\}$. It is easy to see that

$$
\mathcal{D}^{0}=\operatorname{Span}_{\mathbb{Z}_{2}}\left\{\left(1^{16} 0^{32}\right),\left(0^{32} 1^{16}\right),(\alpha, \alpha, \alpha) \mid \begin{array}{cc}
\alpha=\left(1^{16}\right), & \left(\left\{1^{4} 0^{4}\right\}^{2}\right), \\
\left(\left\{1^{2} 0^{2}\right\}^{4}\right) & \text { or }\left(\{10\}^{8}\right)
\end{array}\right\}
$$

and $\mathcal{D}^{1}=\left(\left\{1^{8} 0^{8}\right\}^{3}\right)+\mathcal{D}^{0}$. Therefore, the index $\left[\mathcal{D}: \mathcal{D}^{0}\right]$ is 2 and in this case the involution $\sigma_{\xi} \in \operatorname{Aut}\left(\left(V^{\natural}\right)^{0}\right)$ can be extended to an automorphism $\theta_{\xi} \in \operatorname{Stab}_{V^{\natural}}^{\mathrm{pt}}(F)$ of order 4 by Theorem 6.7. We also set $\mathcal{C}^{0}:=\{\alpha \in \mathcal{C} \mid\langle\alpha, \xi\rangle=0\}$ and $\mathcal{C}^{1}:=\{\alpha \in \mathcal{C} \mid\langle\alpha, \xi\rangle=1\}$. Let us consider a subgroup of $\mathcal{D} \times \mathbb{C}^{*}$ defined by

$$
\tilde{\mathcal{D}}:=\left(\mathcal{D}^{0} \times\{ \pm 1\}\right) \sqcup\left(\mathcal{D}^{1} \times\{ \pm \sqrt{-1}\}\right) .
$$


For $(\alpha, u) \in \mathcal{D} \times \mathbb{C}^{*}$, set $\left(V^{\natural}\right)^{(\alpha, u)}:=\left\{x \in\left(V^{\natural}\right)^{\alpha} \mid \theta_{\xi} x=u x\right\}$. Then we have a $\tilde{\mathcal{D}}$-graded decomposition

$$
V^{\natural}=\bigoplus_{(\alpha, u) \in \tilde{\mathcal{D}}}\left(V^{\natural}\right)^{(\alpha, u)}, \quad\left(V^{\natural}\right)^{(0,1)}=V_{\mathcal{C}^{0}}, \quad\left(V^{\natural}\right)^{(0,-1)}=V_{\mathcal{C}^{1}},
$$

where $V_{\mathcal{C}^{0}}$ denotes the code VOA associated to $\mathcal{C}^{0}$ and $V_{\mathcal{C}^{1}}$ is its module. Since $\left(\mathcal{C}^{0}\right)_{\alpha}$ contains a self-dual subcode w.r.t. $\alpha \in \mathcal{D}$ by Lemma 6.5, all $\left(V^{\natural}\right)^{(\alpha, u)},(\alpha, u) \in \tilde{\mathcal{D}}$, are

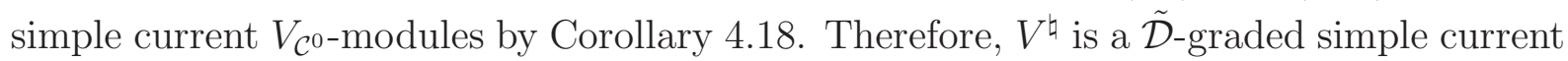
extension of a code $\operatorname{VOA}\left(V^{\natural}\right)^{(0,1)}=V_{\mathcal{C}^{0}}$.

By direct computation, it is not difficult to obtain the following lemma.

Lemma 7.3. For any non-zero $\alpha \in \mathcal{D}^{0}$, the subset $\left(\mathcal{C}^{1}\right)_{\alpha}$ is not empty. In other words, $\left[\mathcal{C}_{\alpha},\left(\mathcal{C}^{0}\right)_{\alpha}\right]=2$.

Remark 7.4. For a general framed VOA with structure codes $(C, D)$, it is possible that the set $\left(C^{1}\right)_{\alpha}$ is empty for some non-zero $\alpha \in D$. For example, we can take $D=$ $\left\{\left(0^{16}\right),\left(1^{8} 0^{8}\right),\left(0^{8} 1^{8}\right),\left(1^{16}\right)\right\}, C=D^{\perp}, \xi=\left(1^{2} 0^{14}\right)$ and $\alpha=\left(0^{8} 1^{8}\right)$. Then, $C_{\alpha}=\left(C^{0}\right)_{\alpha}$ and $\left(C^{1}\right)_{\alpha}$ is empty. Note that $(C, D)$ can be realized as the structure codes of the lattice $V O A V_{E_{8}}(c f .[\underline{D G H}])$.

Theorem 7.5. $\theta_{\xi}$ is a 4 A-element of the Monster.

Proof: We shall compute the McKay-Thompson series of $\theta_{\xi}$ defined by

$$
T_{\theta_{\xi}}(z):=\operatorname{tr}_{V \natural} \theta_{\xi} q^{L(0)-1}, \quad q=e^{2 \pi \sqrt{-1} z} .
$$

Recall the notion of the conformal character of a module $M=\oplus_{n \geq 0} M_{n+h}$ over a VOA $V$ :

$$
\operatorname{ch}_{M}(q):=\operatorname{tr}_{M} q^{L(0)-c / 24}=\sum_{n=0}^{\infty} \operatorname{dim}_{\mathbb{C}} M_{n+h} q^{n+h-c / 24} .
$$

It is clear that

$$
\begin{aligned}
T_{\theta_{\xi}}(z)= & \sum_{\alpha \in \mathcal{D}^{0}} \operatorname{ch}_{\left(V^{\natural}\right)^{(\alpha, 1)}}(q)-\sum_{\alpha \in \mathcal{D}^{0}} \operatorname{ch}_{\left(V^{\natural}\right)^{(\alpha,-1)}}(q) \\
& +\sqrt{-1} \sum_{\alpha \in \mathcal{D}^{1}} \operatorname{ch}_{\left(V^{\natural}\right)^{(\alpha, \sqrt{-1})}}(q)-\sqrt{-1} \sum_{\alpha \in \mathcal{D}^{1}} \operatorname{ch}_{\left(V^{\natural}\right)^{(\alpha,-\sqrt{-1})}}(q) .
\end{aligned}
$$

Let $\alpha \in \mathcal{D}^{1}$. Since $\left(V^{\natural}\right)^{(\alpha, \pm \sqrt{-1})}$ are dual to each other, their conformal characters are the same. Let $\alpha \in \mathcal{D}^{0}$ be a non-zero codeword. By Lemma [7.3, there exists a codeword in $\left(\mathcal{C}^{1}\right)_{\alpha}$. Then by Corollary 4.8 one sees that $\left(V^{\natural}\right)^{(\alpha, 1)}$ and $\left(V^{\natural}\right)^{(\alpha,-1)}$ are isomorphic $F$-modules. Therefore, they have the same conformal characters. Then

$$
\begin{aligned}
T_{\theta_{\xi}}(z) & =\operatorname{ch}_{\left(V^{\natural}\right)(0,1)}(q)-\operatorname{ch}_{\left(V^{\natural}\right)^{(0,-1)}}(q) \\
& =\operatorname{ch}_{V_{\mathcal{C}^{0}}}(q)-\operatorname{ch}_{V_{\mathcal{C}^{1}}}(q) \\
& =2 \operatorname{ch}_{V_{\mathcal{C}^{0}}}(q)-\operatorname{ch}_{V_{\mathcal{C}}}(q) .
\end{aligned}
$$


The conformal character of a code VOA can be easily computed. The following conformal characters are well-known (cf. [FFR]):

$$
\operatorname{ch}_{L(1 / 2,0)}(q) \pm \operatorname{ch}_{L(1 / 2,1 / 2)}(q)=q^{-1 / 48} \prod_{n=0}^{\infty}\left(1 \pm q^{n+1 / 2}\right)
$$

Since $\mathcal{C}=\mathcal{D}^{\perp}$ and $\mathcal{C}^{0}=(\mathcal{D}+\langle\xi\rangle)^{\perp}$, the weight enumerators of these codes are calculated by the MacWilliams identity [McS]:

$$
\begin{aligned}
W_{\mathcal{C}}(x, y) & =\frac{1}{|\mathcal{D}|} W_{\mathcal{D}}(x+y, x-y), \\
W_{\mathcal{C}^{0}}(x, y) & =\frac{1}{|\mathcal{D}+\langle\xi\rangle|} W_{\mathcal{D}+\langle\xi\rangle}(x+y, x-y),
\end{aligned}
$$

where

$$
\begin{aligned}
W_{\mathcal{D}}(x, y)= & x^{48}+3 x^{32} y^{16}+120 x^{24} y^{24}+3 x^{16} y^{32}+y^{48} \\
W_{\mathcal{D}+\langle\xi\rangle}(x, y)= & x^{48}+2 x^{36} y^{12}+3 x^{32} y^{16}+30 x^{28} y^{20}+184 x^{24} y^{24}+30 x^{20} y^{28} \\
& +3 x^{16} y^{32}+2 x^{12} y^{36}+y^{48}
\end{aligned}
$$

Now set

$$
\begin{aligned}
f(x, y) & :=W_{\mathcal{D}+\langle\xi\rangle}(x, y)-W_{\mathcal{D}}(x, y) \\
& =2 x^{36} y^{12}+30 x^{28} y^{20}+64 x^{24} y^{24}+30 x^{20} y^{28}+2 x^{12} y^{36}
\end{aligned}
$$

Then one has

$$
\begin{aligned}
T_{\theta_{\xi}}(z) & =2 \operatorname{ch}_{V_{\mathcal{C}^{0}}}(q)-\operatorname{ch}_{V_{\mathcal{C}}}(q) \\
& =\left[2 W_{\mathcal{C}^{0}}(x, y)-W_{\mathcal{C}}(x, y)\right]_{x=\operatorname{ch}_{L(1 / 2,0)}(q), y=\operatorname{ch}_{L(1 / 2,1 / 2)}(q)} \\
& =\frac{1}{2^{7}}\left[W_{\mathcal{D}+\langle\xi\rangle}(x+y, x-y)-W_{\mathcal{D}}(x+y, x-y)\right]_{\substack{x=\operatorname{ch}_{L(1 / 2,0)}(q), y=\operatorname{ch}_{L(1 / 2,1 / 2)}(q)}} \\
& =\frac{1}{2^{7}} q^{-1} f\left(\prod_{n=0}^{\infty}\left(1+q^{n+1 / 2}\right), \prod_{n=0}^{\infty}\left(1-q^{n+1 / 2}\right)\right) \\
& =q^{-1}+276 q+2048 q^{2}+\cdots
\end{aligned}
$$

Therefore, $\theta_{\xi}$ is a 4 A-element of the Monster by ATLAS].

Next, we shall construct the irreducible $4 \mathrm{~A}$-twisted $V^{\natural}$-module. Let us consider irre-

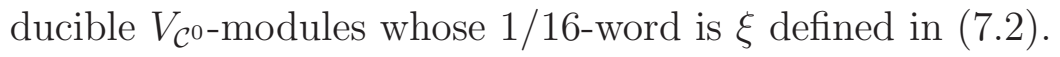

Lemma 7.6. (1) $\left(\mathcal{C}^{0}\right)_{\xi}$ is a self-dual subcode w.r.t. $\xi$.

(2) For any $\gamma \in \mathbb{Z}_{2}^{n}$, the dual of $M_{\mathcal{C}^{0}}(\xi, \gamma ; \iota)$ is isomorphic to $M_{\mathcal{C}^{0}}(\xi, \gamma+\kappa ; \iota)$ with $\kappa=$ $\left(\left\{10^{7}\right\}^{2} 0^{32}\right) \in \mathbb{Z}_{2}^{48}$. 
Proof: By a direct computation, one can show that $\mathcal{C}_{\xi}=\left(\mathcal{C}^{0}\right)_{\xi}$ is generated by the following generator matrix:

$\left[\begin{array}{llllll}11000000 & 11000000 & 00000000 & 00000000 & 00000000 & 00000000 \\ 00000000 & 00000000 & 01100000 & 01100000 & 00000000 & 00000000 \\ 00000000 & 00000000 & 00000000 & 00000000 & 10100000 & 10100000 \\ 10000000 & 10000000 & 01000000 & 01000000 & 00000000 & 00000000 \\ 10000000 & 10000000 & 00000000 & 00000000 & 10000000 & 10000000 \\ 11000000 & 00000000 & 01100000 & 00000000 & 10100000 & 00000000\end{array}\right]$

From this, it is easy to see that $\left(\mathcal{C}^{0}\right)_{\xi}$ is a self-dual code w.r.t. $\xi$. If we set

$$
\kappa=(100000001000000000000000000000000000000000000000) \in \mathbb{Z}_{2}^{48},
$$

then $\operatorname{supp}(\kappa) \subset \operatorname{supp}(\xi)$ and we have $(-1)^{\langle\alpha, \kappa\rangle}=(-1)^{\mathrm{wt}(\alpha) / 2}$ for all $\alpha \in\left(\mathcal{C}^{0}\right)_{\xi}$. Therefore, the dual of $M_{\mathcal{C}^{0}}(\xi, \gamma ; \iota)^{*}$ is isomorphic to $M_{\mathcal{C}^{0}}(\xi, \gamma+\kappa ; \iota)$ by Proposition 4.11,

By the lemma above, we know that $\left(\mathcal{C}^{0}\right)_{\xi}$ is self-dual w.r.t. $\xi$ and thus $\left(\mathcal{C}^{0}\right)_{\xi}$ equals to its own radical. From now on, we shall fix a map $\iota:\left(\mathcal{C}^{0}\right)_{\xi} \rightarrow \mathbb{C}^{*}$ such that the section map $\left(\mathcal{C}^{0}\right)_{\xi} \ni \alpha \mapsto(\alpha, \iota(\alpha)) \in \pi_{\mathbb{C}^{*}}^{-1}\left(\mathcal{C}^{0}\right)$ is a group homomorphism. We shall simply denote $M_{\mathcal{C}^{0}}(\xi, \gamma ; \iota)$ by $M_{\mathcal{C}^{0}}(\xi, \gamma)$ and set $W:=M_{\mathcal{C}^{0}}(\xi, 0)$.

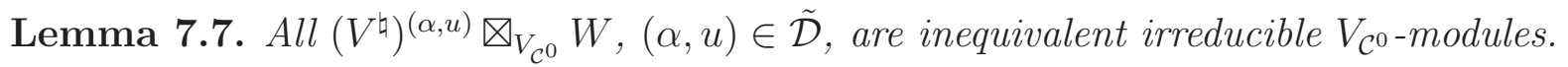

Proof: Suppose $\left(V^{\natural}\right)^{(\alpha, u)} \bigotimes_{V_{\mathcal{C}^{0}}} W \simeq\left(V^{\natural}\right)^{(\beta, v)} \bigotimes_{V_{\mathcal{C}^{0}}} W$ with $(\alpha, u),(\beta, v) \in \tilde{\mathcal{D}}$. Since $\left(\left(V^{\natural}\right)^{(\alpha, u)}\right)^{*} \simeq\left(V^{\natural}\right)^{(\alpha, u)^{-1}}=\left(V^{\natural}\right)^{\left(\alpha, u^{-1}\right)}$, we have

$$
\begin{aligned}
W & =\left(V^{\natural}\right)^{\left(\alpha, u^{-1}\right)} \bigotimes_{V_{\mathcal{C}^{0}}}\left(V^{\natural}\right)^{(\alpha, u)} \bigotimes_{V_{\mathcal{C}^{0}}} W \\
& =\left(V^{\natural}\right)^{\left(\alpha, u^{-1}\right)} \otimes_{V_{\mathcal{C}^{0}}}\left(V^{\natural}\right)^{(\beta, v)} \bigotimes_{V_{\mathcal{C}^{0}}} W \\
& =\left(V^{\natural}\right)^{\left(\alpha+\beta, u^{-1} v\right)} \bigotimes_{V_{\mathcal{C}^{0}}} W
\end{aligned}
$$

in the fusion algebra. By considering 1/16-word decompositions, one has $\alpha=\beta$ and $u^{-1} v \in\{ \pm 1\}$. Let $\delta \in \mathcal{C}$ be such that $\mathcal{C}^{1}=\mathcal{C}^{0}+\delta$. If $u^{-1} v=-1$, then $\left(V^{\natural}\right)^{\left(\alpha+\beta, u^{-1} v\right)}=$ $\left(V^{\natural}\right)^{(0,-1)}=V_{\mathcal{C}^{1}}=M_{\mathcal{C}^{0}}(0, \delta)$ so that by Lemma 4.14 one has

$$
W=\left(V^{\natural}\right)^{\left(\alpha+\beta, u^{-1} v\right)} \underset{V_{\mathcal{C}^{0}}}{\bigotimes} W=M_{\mathcal{C}^{0}}(0, \delta) \underset{V_{\mathcal{C}^{0}}}{\bigotimes} M_{\mathcal{C}^{0}}(\xi, 0)=M_{\mathcal{C}^{0}}(\xi, \delta) .
$$

It is shown in Lemma 7.6 that $\left(\mathcal{C}^{0}\right)_{\xi}$ contains a self-dual subcode w.r.t. $\xi$. Then $W$ is not isomorphic to $M_{\mathcal{C}^{0}}(\xi, \delta)$ by Lemma 4.7 and we obtain a contradiction. Therefore, $u^{-1} v=1$ and hence $(\alpha, u)=(\beta, v)$. This completes the proof.

By Theorem 2.4 and the lemma above, the space

$$
V^{\natural}\left(\theta_{\xi}\right):=V^{\natural} \underset{V_{\mathcal{C}^{0}}}{\otimes} W=\bigoplus_{(\alpha, u) \in \tilde{\mathcal{D}}}\left(V^{\natural}\right)^{(\alpha, u)} \underset{\left(V^{\natural}\right)^{(0,1)}}{\bigotimes} M_{\mathcal{C}^{0}}(\xi, 0)
$$


carries a unique structure of an irreducible $\chi_{W}$-twisted $V^{\natural}$-module for some $\chi_{W} \in(\tilde{\mathcal{D}})^{*} \subset$ M. It is clear that $\chi_{W} \in \operatorname{Stab}_{V^{\natural}}^{\mathrm{pt}}(F)$. Since the top weight of $W$ is $3 / 4, V^{\natural}\left(\theta_{\xi}\right)$ is neither 2A-twisted nor $2 \mathrm{~B}$-twisted $V^{\natural}$-module so that $\chi_{W}$ is an element of $\mathbb{M}$ of order 4 . Hence, there exists $\xi^{\prime} \in \mathcal{P}$ such that $\left.\chi_{W}^{2}\right|_{\left(V^{\natural}\right)^{0}}=\sigma_{\xi^{\prime}}$. By the construction of $V^{\natural}\left(\theta_{\xi}\right)$, we know that $\mathcal{C}^{0} \subset\left\{\alpha \in \mathcal{C} \mid\left\langle\alpha, \xi^{\prime}\right\rangle=0\right\} \subsetneq \mathcal{C}$ so that $\sigma_{\xi}=\sigma_{\xi^{\prime}}$ and $\chi_{W}=\theta_{\xi} \cdot \tau_{\eta}$ for some $\eta \in \mathbb{Z}_{2}^{48}$. Since the definition of $\theta_{\xi}$ is only unique up to a product of $\tau$-involutions, we can take $\theta_{\xi}=\chi_{W}$. Note that the definition of $V^{\natural}\left(\theta_{\xi}\right)$ in (7.6) depends only on the decomposition

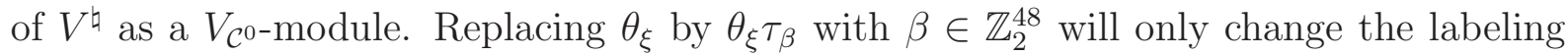
of the $V_{\mathcal{C}^{0} \text {-modules in (7.4) }}$ and does not affect the isotypical decomposition of $V^{\natural}$ as a

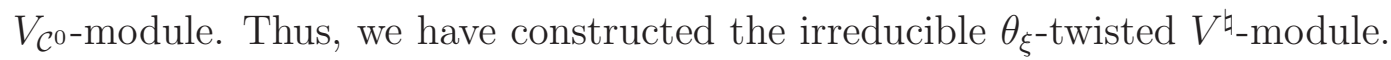

Theorem 7.8. $V^{\natural}\left(\theta_{\xi}\right)$ defined in (7.6) is an irreducible 4 A-twisted module over $V^{\natural}$.

Remark 7.9. By Lemma 7.6 and Corollary 4.8, we see that the top weight of the 4Atwisted module is $3 / 4$ and the dimension of the top level is 1.

Let us consider the dual module $W^{*}$ of $W$. It is clear that $W$ and $W^{*}$ are inequivalent

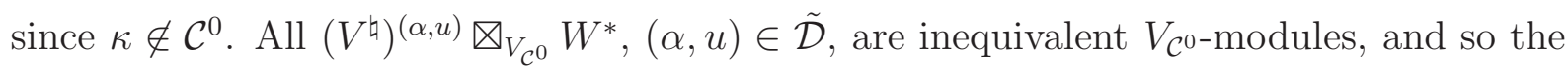
space

$$
V^{\natural}\left(\theta_{\xi}^{3}\right):=V^{\natural} \underset{V_{\mathcal{C}^{0}}}{\bigotimes} W^{*}=\bigoplus_{(\alpha, u) \in \tilde{\mathcal{D}}}\left(V^{\natural}\right)^{(\alpha, u)} \underset{\left(V^{\natural}\right)^{(0,1)}}{\bigotimes} M_{\mathcal{C}^{0}}(\xi, \kappa)
$$

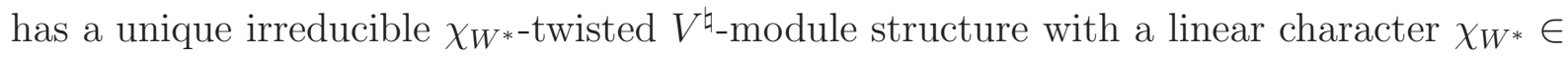
$\tilde{\mathcal{D}}^{*}$ (cf. Theorem 2.4). It is also shown DLM2 that the dual of $\chi_{W^{-}}$-twisted module forms a $\chi_{W}^{-1}$-twisted module. The dual $V^{\natural}$-module of $V^{\natural}\left(\theta_{\xi}\right)$ contains $W^{*}$ as a $V_{\mathcal{C}^{0} \text {-submodule, }}$ and $V^{\natural}\left(\theta_{\xi}^{3}\right)$ is uniquely determined by $W^{*}$, so $\chi_{W^{*}}$ is actually equal to $\theta_{\xi}^{3}=\theta_{\xi}^{-1}$ by our choice of $\theta_{\xi}$. Therefore, $V^{\natural}\left(\theta_{\xi}^{3}\right)$ is the irreducible $\theta_{\xi}^{3}$-twisted $V^{\natural}$-module.

In order to perform the $4 \mathrm{~A}$-twisted orbifold construction of $V^{\natural}$, we classify the irreducible representations of $\left(V^{\natural}\right)^{\left\langle\theta_{\xi}\right\rangle}$. By (7.4), the fixed point subalgebra $\left(V^{\natural}\right)^{\left\langle\theta_{\xi}\right\rangle}$ is a framed VOA with structure codes $\left(\mathcal{C}^{0}, \mathcal{D}^{0}\right)$.

Proposition 7.10. There are 16 inequivalent irreducible $\left(V^{\natural}\right)^{\left\langle\theta_{\xi}\right\rangle}$-modules. Every irreducible $\left(V^{\natural}\right)^{\left\langle\theta_{\xi}\right\rangle}$-module is a submodule of an irreducible $\theta_{\xi}^{i}$-twisted $V^{\natural}$-module for $0 \leq i \leq$ 3. Among them, 8 irreducible modules have integral top weights.

Proof: Since $V^{\natural}$ and $\left(V^{\natural}\right)^{\left\langle\theta_{\xi}\right\rangle}$ are simple current extensions of the code VOA $V_{\mathcal{C}^{0}}, V^{\natural}$ is a $\mathbb{Z}_{4}$-graded simple current extension of $\left(V^{\natural}\right)^{\left\langle\theta_{\xi}\right\rangle}$ by Proposition 2.3. Then by Theorem 2.4, every irreducible $\left(V^{\natural}\right)^{\left\langle\theta_{\xi}\right\rangle}$-module is a submodule of a $\theta_{\xi}^{i}$-twisted module for $0 \leq i \leq 3$. It is shown in [DLM3] that $V^{\natural}$ has a unique irreducible $\theta_{\xi^{i}}^{i}$ twisted module for $0 \leq i \leq 3$ as $V^{\natural}$ is holomorphic. Therefore, we only have to show that each irreducible $\theta_{\xi}^{i}$-twisted $V^{\natural}$-module decomposes into a direct sum of 4 inequivalent irreducible $\left(V^{\natural}\right)^{\left\langle\theta_{\xi}\right\rangle}$-submodules. Since $V^{\natural}\left(\theta_{\xi}\right)$ and $V^{\natural}\left(\theta_{\xi}^{3}\right)$ are dual to each other (cf. [DLM2]), we know each of them has four 


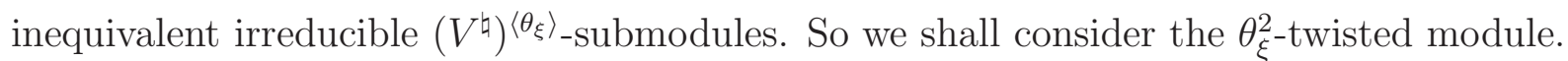
Let $\kappa \in \mathbb{Z}_{2}^{48}$ be defined as in (7.5). Then one can easily verify that $\theta_{\xi}^{2}=\tau_{\kappa}$. We consider an irreducible $V_{\mathcal{C}^{0}}$-module $M_{\mathcal{C}^{0}}(0, \kappa)$. We claim that $\left(V^{\natural}\right)^{(\alpha, u)} \otimes_{V_{\mathcal{C}^{0}}} M_{\mathcal{C}^{0}}(0, \kappa),(\alpha, u) \in \tilde{\mathcal{D}}$, are inequivalent irreducible $V_{\mathcal{C}^{0}}$-modules. The irreducibility of $\left(V^{\natural}\right)^{(\alpha, u)} \bigotimes_{V_{\mathcal{C}^{0}}} M_{\mathcal{C}^{0}}(0, \kappa)$

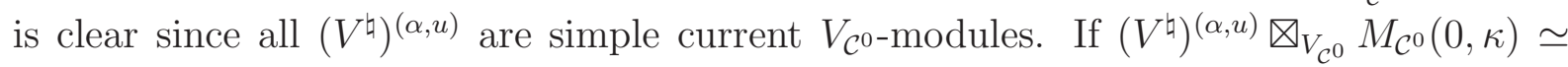
$\left(V^{\natural}\right)^{(\beta, v)} \otimes_{V_{\mathcal{C}^{0}}} M_{\mathcal{C}^{0}}(0, \kappa)$, then by the 1/16-word decomposition, we have $\alpha=\beta$ and $u^{-1} v \in$ $\{ \pm 1\}$. Let $\delta \in \mathbb{Z}_{2}^{48}$ such that $\mathcal{C}^{1}=\mathcal{C}^{0}+\delta$. If $u^{-1} v=-1$, then $\left(V^{\natural}\right)^{\left(\alpha+\beta, u^{-1} v\right)}=M_{\mathcal{C}^{0}}(0, \delta)$ and one has

$$
\begin{aligned}
M_{\mathcal{C}^{0}}(0, \kappa) & =\left(V^{\natural}\right)^{\left(\alpha, u^{-1}\right)} \bigotimes_{V_{\mathcal{C}^{0}}}\left(V^{\natural}\right)^{(\beta, v)} \bigotimes_{V_{\mathcal{C}^{0}}} M_{\mathcal{C}^{0}}(0, \kappa) \\
& =\left(V^{\natural}\right)^{\left(\alpha+\beta, u^{-1} v\right)} \bigotimes_{V_{\mathcal{C}^{0}}} M_{\mathcal{C}^{0}}(0, \kappa) \\
& =M_{\mathcal{C}^{0}}(0, \delta) \bigotimes_{V_{\mathcal{C}^{0}}} M_{\mathcal{C}^{0}}(0, \kappa) \\
& =M_{\mathcal{C}^{0}}(0, \kappa+\delta) .
\end{aligned}
$$

But this is a contradiction by Lemma 4.7. Therefore, all $\left(V^{\natural}\right)^{(\alpha, u)} \bigotimes_{V_{\mathcal{C}^{0}}} M_{\mathcal{C}^{0}}(0, \kappa),(\alpha, u) \in$ $\tilde{\mathcal{D}}$, are inequivalent irreducible $V_{\mathcal{C}^{0}}$-modules. Now by Theorem 2.4 and Lemma 4.14 the space

$$
V^{\natural}\left(\theta_{\xi}^{2}\right):=\bigoplus_{(\alpha, u) \in \tilde{\mathcal{D}}}\left(V^{\natural}\right)^{(\alpha, u)} \underset{V_{\mathcal{C}^{0}}}{\otimes} M_{\mathcal{C}^{0}}(0, \kappa)
$$

forms an irreducible $\tau_{\kappa}=\theta_{\xi}^{2}$-twisted $V^{\natural}$-module. Thus $V^{\natural}\left(\theta_{\xi}^{2}\right)$ splits into four irreducible $\left.\left(V^{\natural}\right)^{\left\langle\theta_{\xi}\right.}\right\rangle_{\text {-submodules as follows. }}$

$$
\begin{aligned}
& V^{\natural}\left(\theta_{\xi}^{2}\right)=\left\{\bigoplus_{\alpha \in \mathcal{D}^{0}}\left(V^{\natural}\right)^{(\alpha, 1)} \underset{V_{\mathcal{C}^{0}}}{\otimes} M_{\mathcal{C}^{0}}(0, \kappa)\right\} \bigoplus\left\{\bigoplus_{\alpha \in \mathcal{D}^{0}}\left(V^{\natural}\right)^{(\alpha,-1)} \underset{V_{\mathcal{C}^{0}}}{\otimes} M_{\mathcal{C}^{0}}(0, \kappa)\right\} \\
& \bigoplus\left\{\bigoplus_{\alpha \in \mathcal{D}^{1}}\left(V^{\natural}\right)^{(\alpha, \sqrt{-1})} \underset{V_{\mathcal{C}^{0}}}{\otimes} M_{\mathcal{C}^{0}}(0, \kappa)\right\} \bigoplus\left\{\bigoplus_{\alpha \in \mathcal{D}^{1}}\left(V^{\natural}\right)^{(\alpha,-\sqrt{-1})} \underset{V_{\mathcal{C}^{0}}}{\otimes} M_{\mathcal{C}^{0}}(0, \kappa)\right\} .
\end{aligned}
$$

Therefore, all irreducible $\theta_{\xi}^{i}$-twisted $V^{\natural}$-modules are direct sums of 4 inequivalent irreducible $\left(V^{\natural}\right)^{\left\langle\theta_{\xi}\right\rangle}$-submodules and we have obtained 16 irreducible $\left(V^{\natural}\right)^{\left\langle\theta_{\xi}\right\rangle}$-modules. It remains to show that these 16 modules are inequivalent. Since every irreducible $\left(V^{\natural}\right)^{\left\langle\theta_{\xi}\right\rangle}$ module can be uniquely extended to a $\theta_{\xi}^{i}$-twisted $V^{\natural}$-module by Theorem 2.4 these 16 irreducible modules are actually inequivalent.

Among these 16 irreducible $\left.\left(V^{\natural}\right)^{\left\langle\theta_{\xi}\right.}\right\rangle$-modules, we have 4 modules having integral top weights from $V^{\natural}, 2$ from $\theta_{\xi}^{2}$-twisted $V^{\natural}$-module, 1 from $\theta_{\xi^{-}}$-twisted and 1 from $\theta_{\xi^{3}}^{3}$-twisted modules, respectively. This completes the proof.

We have also shown that every irreducible $\theta_{\xi}^{i}$-twisted $V^{\natural}$-module has a $\mathbb{Z}_{4}$-grading which agrees with the action of $\theta_{\xi}$ on $V^{\natural}$. By this fact, we adopt the following notation.

For $u \in \mathbb{C}^{*}$ satisfying $u^{4}=1$, we set $V^{\natural}(1, u):=\left\{a \in V^{\natural} \mid \theta_{\xi} a=u a\right\}$. For $i=1$ or 3, we define $V^{\natural}\left(\theta_{\xi}^{i}, 1\right)$ to be the unique irreducible $\left(V^{\natural}\right)^{\left\langle\theta_{\xi}\right\rangle}$-submodule of $V^{\natural}\left(\theta_{\xi}^{i}\right)$ which has 
an integral top weight. They can be defined explicitly as follows.

$$
\begin{aligned}
& V^{\natural}\left(\theta_{\xi}, 1\right):=\bigoplus_{\alpha \in \mathcal{D}^{1}}\left(V^{\natural}\right)^{(\alpha,-\sqrt{-1})} \bigotimes_{V_{\mathcal{C}^{0}}} M_{\mathcal{C}^{0}}(\xi, 0), \\
& V^{\natural}\left(\theta_{\xi}^{3}, 1\right):=\bigoplus_{\alpha \in \mathcal{D}^{1}}\left(V^{\natural}\right)^{(\alpha, \sqrt{-1})} \bigotimes_{V_{\mathcal{C}^{0}}} M_{\mathcal{C}^{0}}(\xi, \kappa) .
\end{aligned}
$$

For $i=2$, there are two irreducible $\left(V^{\natural}\right)^{\left\langle\theta_{\xi}\right\rangle}$-submodules in $V^{\natural}\left(\theta_{\xi}^{2}\right)$ having integral top weights. We shall define

$$
\begin{aligned}
& V^{\natural}\left(\theta_{\xi}^{2}, 1\right):=\bigoplus_{\alpha \in \mathcal{D}^{0}}\left(V^{\natural}\right)^{(\alpha,-1)} \bigotimes_{V_{\mathcal{C}^{0}}} M_{\mathcal{C}^{0}}(0, \kappa), \\
& V^{\natural}\left(\theta_{\xi}^{2},-1\right):=\bigoplus_{\alpha \in \mathcal{D}^{0}}\left(V^{\natural}\right)^{(\alpha, 1)} \bigotimes_{V_{\mathcal{C}^{0}}} M_{\mathcal{C}^{0}}(0, \kappa) .
\end{aligned}
$$

In addition, we define

$$
V^{\natural}\left(\theta_{\xi}^{i}, u\right):=V^{\natural}(1, u) \underset{\left(V^{\natural}\right)^{\left\langle\theta_{\xi}\right\rangle}}{\bigotimes} V^{\natural}\left(\theta_{\xi}^{i}, 1\right) \quad \text { for } 1 \leq i \leq 3 .
$$

Set $G:=\left\langle\theta_{\xi}\right\rangle \times\left\{u \in \mathbb{C}^{*} \mid u^{4}=1\right\}$. Then $\left\{V^{\natural}(g, u) \mid(g, u) \in G\right\}$ is the set of all inequivalent irreducible $\left(V^{\natural}\right)^{\left\langle\theta_{\xi}\right\rangle}$-modules.

Proposition 7.11. The fusion algebra associated to $\left(V^{\natural}\right)^{\left\langle\theta_{\xi}\right\rangle}$ is isomorphic to the group algebra of $G$. The isomorphism is given by $V^{\natural}(g, u) \mapsto(g, u)$.

Proof: Since the structure codes of $\left(V^{\natural}\right)^{\left\langle\theta_{\xi}\right\rangle}$ is $\left(\mathcal{C}^{0}, \mathcal{D}^{0}\right),\left(V^{\natural}\right)^{\left\langle\theta_{\xi}\right\rangle}$ is a $\mathcal{D}^{0}$-graded simple current extension of $V_{\mathcal{C}^{0}}$. So we have the following fusion rules:

$$
V^{\natural}(1, u) \underset{\left(V^{\natural}\right)^{\left\langle\theta_{\xi}\right\rangle}}{\bigotimes} V^{\natural}(1, v)=V^{\natural}(1, u v) \quad \text { for } u, v \in \mathbb{C}^{*}, u^{4}=v^{4}=1 .
$$

Since all $V^{\natural}(g, u),(g, u) \in G$, are $\mathcal{D}^{0}$-stable, we can use Proposition 2.5. The following fusion rules of $V_{\mathcal{C}^{0}}$-modules are already known:

$$
\begin{array}{ll}
M_{\mathcal{C}^{0}}(0, \kappa) \bigotimes_{V_{\mathcal{C}^{0}}} M_{\mathcal{C}^{0}}(0, \kappa)=M_{\mathcal{C}^{0}}(0,0), & M_{\mathcal{C}^{0}}(0, \kappa) \bigotimes_{V_{\mathcal{C}^{0}}} M_{\mathcal{C}^{0}}(\xi, 0)=M_{\mathcal{C}^{0}}(\xi, \kappa), \\
M_{\mathcal{C}^{0}}(\xi, 0) \bigotimes_{V_{\mathcal{C}^{0}}} M_{\mathcal{C}^{0}}(\xi, 0)=M_{\mathcal{C}^{0}}(0, \kappa), & M_{\mathcal{C}^{0}}(\xi, 0) \bigotimes_{V_{\mathcal{C}^{0}}} M_{\mathcal{C}^{0}}(\xi, \kappa)=M_{\mathcal{C}^{0}}(0,0) .
\end{array}
$$

Therefore, we have the following fusion rules of $\left(V^{\natural}\right)^{\left\langle\theta_{\xi}\right\rangle}$-modules:

$$
\begin{array}{ll}
V^{\natural}\left(\theta^{2}, 1\right) \bigotimes_{\left(V^{\natural}\right)^{\left\langle\theta_{\xi}\right\rangle}} V^{\natural}\left(\theta^{2}, 1\right)=V^{\natural}(1,1), & V^{\natural}\left(\theta^{2}, 1\right) \bigotimes_{\left(V^{\natural}\right)^{\left\langle\theta_{\xi}\right\rangle}} V^{\natural}(\theta, 1)=V^{\natural}\left(\theta^{3}, 1\right), \\
V^{\natural}(\theta, 1) \bigotimes_{\left(V^{\natural}\right)^{\left\langle\theta_{\xi}\right\rangle}} V^{\natural}(\theta, 1)=V^{\natural}\left(\theta^{2}, 1\right), & V^{\natural}(\theta, 1) \bigotimes_{\left(V^{\natural}\right)^{\left\langle\theta_{\xi}\right\rangle}} V^{\natural}\left(\theta^{3}, 1\right)=V^{\natural}(1,1) .
\end{array}
$$

Since the fusion algebra is commutative and associative, the remaining fusion rules are deduced from the above and we can establish the isomorphism.

A $\theta_{\xi}$-twisted orbifold construction of $V^{\natural}$ refers to a construction of a $\mathbb{Z}_{4}$-graded (simple current) extension of the $\theta_{\xi^{-}}$-fixed point subalgebra $\left(V^{\natural}\right)^{\left\langle\theta_{\xi}\right\rangle}$ by using the irreducible submodules of $V^{\natural}\left(\theta_{\xi}^{i}\right)$ with integral weights. By Proposition 7.10, such modules are denoted by

$$
V^{\natural}(1, \pm 1), \quad V^{\natural}(1, \pm \sqrt{-1}), \quad V^{\natural}\left(\theta_{\xi}^{2}, \pm 1\right), \quad V^{\natural}\left(\theta_{\xi}, 1\right) \quad \text { and } \quad V^{\natural}\left(\theta_{\xi}^{3}, 1\right) .
$$


By the fusion rules in Proposition 17.11, there are three possible extensions of $\left(V^{\natural}\right)^{\left\langle\theta_{\xi}\right\rangle}$, namely,

$$
\begin{aligned}
V^{\natural} & =V^{\natural}(1,1) \oplus V^{\natural}(1,-1) \oplus V^{\natural}(1, \sqrt{-1}) \oplus V^{\natural}(1,-\sqrt{-1}), \\
V_{2 B} & =V^{\natural}(1,1) \oplus V^{\natural}(1,-1) \oplus V^{\natural}\left(\theta_{\xi}^{2}, 1\right) \oplus V^{\natural}\left(\theta_{\xi}^{2},-1\right), \\
V_{4 A} & =V^{\natural}(1,1) \oplus V^{\natural}\left(\theta_{\xi}, 1\right) \oplus V^{\natural}\left(\theta_{\xi}^{2}, 1\right) \oplus V\left(\theta_{\xi}^{3}, 1\right) .
\end{aligned}
$$

Consider the fixed point subalgebra $\left(V^{\natural}\right)^{\left\langle\theta_{\xi}^{2}\right\rangle}=V^{\natural}(1,1) \oplus V^{\natural}(1,-1)$. Since $\theta_{\xi}$ is a $4 \mathrm{~A}$ element of $\mathbb{M}$, its square $\theta_{\xi}^{2}$ belongs to the $2 \mathrm{~B}$ conjugacy class of $\mathbb{M}$ [ATLAS]. Therefore, by the original construction of the moonshine VOA in [FLM], the subalgebra $\left(V^{\natural}\right)^{\left\langle\theta_{\xi}^{2}\right\rangle}$ is isomorphic to the fixed point subalgebra $V_{\Lambda}^{+}$of the Leech lattice VOA $V_{\Lambda}$. It is shown in [D] that $V_{\Lambda}^{+}$has four inequivalent irreducible modules which are denoted by $V_{\Lambda}^{ \pm}$and $V_{\Lambda}^{T \pm}$ in [FLM]. Since the top weights of irreducible $V_{\Lambda}^{+}$-modules belong to $\mathbb{Z} / 2$, the inequivalent irreducible $\left(V^{\natural}\right)^{\left\langle\theta_{\xi}^{2}\right\rangle}$-modules are given by the list below:

$$
\begin{array}{ll}
V^{\natural}(1,1) \oplus V^{\natural}(1,-1), & V^{\natural}(1, \sqrt{-1}) \oplus V^{\natural}(1,-\sqrt{-1}), \\
V^{\natural}\left(\theta_{\xi}^{2}, 1\right) \oplus V^{\natural}\left(\theta_{\xi}^{2},-1\right), & V^{\natural}\left(\theta_{\xi}^{2}, \sqrt{-1}\right) \oplus V^{\natural}\left(\theta_{\xi}^{2},-\sqrt{-1}\right) .
\end{array}
$$

It is shown in [H2] that there are two inequivalent simple extensions of $V_{\Lambda}^{+}$; one is the moonshine VOA $V^{\natural}=V_{\Lambda}^{+} \oplus V_{\Lambda}^{T+}$ and the other is $V_{\Lambda}=V_{\Lambda}^{+} \oplus V_{\Lambda}^{-}$. So we have the following isomorphisms:

$$
V_{\Lambda}^{+} \simeq V^{\natural}(1,1) \oplus V^{\natural}(1,-1), \quad V_{\Lambda}^{T+} \simeq V^{\natural}(1, \sqrt{-1}) \oplus V^{\natural}(1,-\sqrt{-1})
$$

We shall prove that $\theta_{\xi}^{2}$-twisted orbifold construction $V_{2 B}$ in (7.11) is isomorphic to the Leech lattice VOA $V_{\Lambda}$. For this, it is enough to show the following.

Lemma 7.12. The top weight of $V^{\natural}\left(\theta_{\xi}^{2},-1\right)$ is 1 and the dimension of the top level is 24 .

Proof: Recall the 1/16-word decomposition (17.9) of $V^{\natural}\left(\theta_{\xi}^{2},-1\right)$ as a module over $V_{\mathcal{C}^{0}}$. It contains a $V_{\mathcal{C}^{0} \text {-submodule }}$

$$
\left(V^{\natural}\right)^{(0,1)} \underset{V_{\mathcal{C}}}{\otimes} M_{\mathcal{C}^{0}}(0, \kappa)=M_{\mathcal{C}^{0}}(0, \kappa)=V_{\mathcal{C}^{0}+\kappa}
$$

By a straightforward computation we see that there are exactly 24 weight two codewords in the coset $\mathcal{C}^{0}+\kappa$ and the support of each is one of the following:

$$
\begin{aligned}
& \{1,9\},\{2,10\},\{3,11\},\{4,12\},\{5,13\},\{6,14\},\{7,15\},\{8,16\}, \\
& \{17,25\},\{18,26\},\{19,27\},\{20,28\},\{21,29\},\{22,30\},\{23,31\},\{24,32\}, \\
& \{33,41\},\{34,42\},\{35,43\},\{36,44\},\{37,45\},\{38,46\},\{39,47\},\{40,48\} .
\end{aligned}
$$

Therefore, the top weight of $V^{\natural}\left(\theta^{2},-1\right)$ is 1 . Then by the list of irreducible $V_{\Lambda}^{+}$-modules in (7.12), the dimension of the top level of $V^{\natural}\left(\theta^{2},-1\right)$ must be 24 . Or, one can directly 
check that all $\left(V^{\natural}\right)^{(\alpha, 1)} \bigotimes_{V_{\mathcal{C}^{0}}} M_{\mathcal{C}^{0}}(0, \kappa)$ has the top weight greater than 1 for any non-zero $\alpha \in \mathcal{D}^{0}$ by considering their $F$-module structures.

Since the top weights of $V_{\Lambda}^{-}$and $V_{\Lambda}^{T-}$ are 1 and $3 / 2$, we have the remaining isomorphisms as follows.

$$
V_{\Lambda}^{-} \simeq V^{\natural}\left(\theta_{\xi}^{2}, 1\right) \oplus V^{\natural}\left(\theta_{\xi}^{2},-1\right), \quad V_{\Lambda}^{T-} \simeq V^{\natural}\left(\theta_{\xi}^{2}, \sqrt{-1}\right) \oplus V^{\natural}\left(\theta_{\xi}^{2},-\sqrt{-1}\right) .
$$

Therefore, the $\theta_{\xi}^{2}$-twisted orbifold $V_{2 B}$ is isomorphic to $V_{\Lambda}$.

Remark 7.13. One can identify $V_{2 B}$ with the Leech lattice VOA without the isomorphisms in (7.13) and (7.15). For, $V_{2 B}$ can be defined as a framed VOA with structure codes $\left(\mathcal{C}^{0} \sqcup \mathcal{C}^{1} \sqcup\left(\mathcal{C}^{0}+\kappa\right) \sqcup\left(\mathcal{C}^{1}+\kappa\right), \mathcal{D}^{0}\right)$ by Theorem 5.11, which is holomorphic by Corollary 5.10. It follows from (7.14) that $V_{2 B}$ contains a free bosonic VOA associated to a vector space of rank 24. Then $V_{2 B}$ is isomorphic to a lattice VOA associated to an even unimodular lattice by [LiX]. Since the weight one subspace of $V_{2 B}$ is 24-dimensional, $V_{2 B}$ is actually isomorphic to the lattice VOA associated to the Leech lattice. Similarly, one can also show that $\left(V^{\natural}\right)^{\left\langle\theta_{\xi}\right\rangle}=V^{\natural}(1,1)$ is isomorphic to a $\mathbb{Z}_{2}$-orbifold $V_{L}^{+}$of a lattice VOA $V_{L}$ for certain sublattice $L$ of $\Lambda$. For, we know that $V^{\natural}(1,1) \oplus V^{\natural}\left(\theta_{\xi}^{2},-1\right)$ forms a sub VOA of $V_{2 B}$ isomorphic to a lattice VOA again by [LiX]. Since $V^{\natural}(1,1)$ is a $\mathbb{Z}_{2}$-fixed point subalgebra of $V^{\natural}(1,1) \oplus V^{\natural}\left(\theta_{\xi}^{2},-1\right)$ under an involution acting on $V^{\natural}\left(\theta_{\xi}^{2},-1\right)$ by -1 , we have the isomorphism as claimed. This isomorphism is first pointed out by Shimakura from a different view point.

Next we consider the proper $\theta_{\xi}$-twisted orbifold construction $V_{4 A}$ in (17.11). Let $\alpha \in \tilde{\mathcal{D}}^{1}$

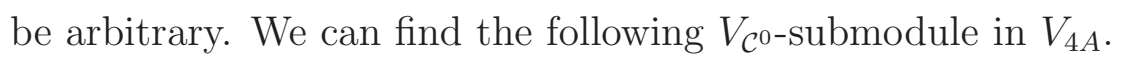

$$
U:=V_{\mathcal{C}^{0}} \oplus V_{\mathcal{C}^{1}+\kappa} \oplus\left\{\left(V^{\natural}\right)^{(\alpha,-\sqrt{-1})} \underset{V_{\mathcal{C}^{0}}}{\bigotimes} M_{\mathcal{C}^{0}}(\xi, 0)\right\} \oplus\left\{\left(V^{\natural}\right)^{(\alpha, \sqrt{-1})} \underset{V_{\mathcal{C}^{0}}}{\bigotimes} M_{\mathcal{C}^{0}}(\xi, \kappa)\right\} .
$$

Lemma 7.14. There exists a unique structure of a framed VOA on $U$.

Proof: Set $U^{0}:=V_{\mathcal{C}^{0}} \oplus V_{\mathcal{C}^{1}+\kappa}$ and

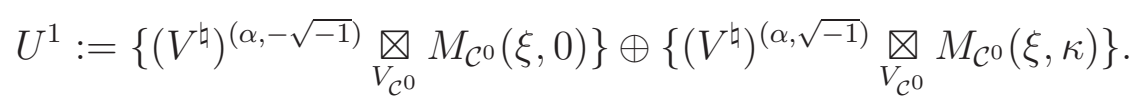

Then $U^{0}$ is a code VOA associated to $\mathcal{C}^{0} \sqcup\left(\mathcal{C}^{1}+\kappa\right)$ and $U^{1}$ is an irreducible $U^{0}$-module with the $1 / 16$-word $\alpha+\xi$. Clearly the top weight of $U^{1}$ is integral. The dual code of $\mathcal{C}^{0} \sqcup\left(\mathcal{C}^{1}+\kappa\right)$ is given by $\mathcal{D}^{0} \sqcup\left(\mathcal{D}^{1}+\xi\right)$ and it is straightforward to verify that $\mathcal{D}^{0} \sqcup\left(\mathcal{D}^{1}+\xi\right)$ is triply even, i.e., $\operatorname{wt}(\alpha)$ is divisible by 8 for any $\alpha \in \mathcal{D}^{0} \sqcup\left(\mathcal{D}^{1}+\xi\right)$. Therefore, $\left(\mathcal{C}^{0} \sqcup\left(\mathcal{C}^{1}+\kappa\right)\right)_{\alpha+\xi}$ contains a doubly even self-dual subcode w.r.t. $\alpha+\xi$ by Remark 5.13. Then by Lemma 5.1, $U=U^{0} \oplus U^{1}$ forms a framed VOA. 
By the lemma above, we can apply the extension property of simple current extensions in Theorem 2.7 to define a framed VOA structure on $V_{4 A}$ with structure codes $\left(\mathcal{C}^{0} \sqcup\left(\mathcal{C}^{1}+\right.\right.$ $\left.\kappa), \mathcal{D}^{0} \sqcup\left(\mathcal{D}^{1}+\xi\right)\right)$. We know that $V_{4 A}$ is holomorphic by Corollary 5.10, We shall prove that $V_{4 A}$ is isomorphic to the moonshine VOA $V^{\natural}$. On $V_{2 B}=V^{\natural}(1,1) \oplus V^{\natural}(1,-1) \oplus$ $V^{\natural}\left(\theta_{\xi}^{2}, 1\right) \oplus V^{\natural}\left(\theta_{\xi}^{2},-1\right)$, define $\psi_{1}, \psi_{2} \in \operatorname{Aut}\left(V_{2 B}\right)$ by

$$
\psi_{1}:=\left\{\begin{array}{c}
1 \text { on } V^{\natural}(1,1) \oplus V^{\natural}(1,-1), \\
-1 \text { on } V^{\natural}\left(\theta_{\xi}^{2}, 1\right) \oplus V^{\natural}\left(\theta_{\xi}^{2},-1\right),
\end{array}\right.
$$

and

$$
\psi_{2}:=\left\{\begin{array}{c}
1 \text { on } V^{\natural}(1,1) \oplus V^{\natural}\left(\theta_{\xi}^{2}, 1\right), \\
-1 \text { on } V^{\natural}(1,-1) \oplus V^{\natural}\left(\theta_{\xi}^{2},-1\right) .
\end{array}\right.
$$

Then both of $\psi_{1}, \psi_{2}$ are involutions on $V_{2 B}$ by the fusion rules in Proposition 7.11 ,

Lemma 7.15. The fixed point subalgebras $V_{2 B}^{\left\langle\psi_{1}\right\rangle}$ and $V_{2 B}^{\left\langle\psi_{2}\right\rangle}$ are isomorphic to the $\mathbb{Z}_{2}$ orbifold subalgebra $V_{\Lambda}^{+}$of the Leech lattice VOA.

Proof: We have shown that $V_{2 B}$ is isomorphic to the Leech lattice VOA $V_{\Lambda}$. By (7.13), the fixed point subalgebra $V_{2 B}^{\left\langle\psi_{1}\right\rangle}$ is isomorphic to the $\mathbb{Z}_{2}$-orbifold $V_{\Lambda}^{+}$. So it remains to prove that $V_{2 B}^{\left\langle\psi_{2}\right\rangle}$ is isomorphic to $V_{2 B}^{\left\langle\psi_{1}\right\rangle}$. Since the weight one subspace $\left(V_{2 B}\right)_{1}$ of $V_{2 B}$ is a subspace of $V^{\natural}\left(\theta^{2},-1\right)$ by Lemma 7.12, both $\psi_{1}$ and $\psi_{2}$ acts as -1 on $\left(V_{2 B}\right)_{1}$. The weight one subspace of $V_{2 B}$ generates a sub VOA isomorphic to the free bosonic VOA $M_{\mathbb{C} \Lambda}(0)$ associated to the linear space $\mathbb{C} \Lambda=\mathbb{C} \otimes_{\mathbb{Z}} \Lambda$. Since $\psi_{1} \psi_{2}^{-1}$ trivially acts on the weight one subspace of $V_{2 B}, \psi_{1} \psi_{2}^{-1}$ commute with the action of $M_{\mathbb{C} \Lambda}(0)$ on $V_{2 B}$. Therefore, $\psi_{1} \psi_{2}^{-1}$ is a linear character $\rho_{h}=\exp \left(2 \pi \sqrt{-1} h_{(0)}\right) \in \operatorname{Aut}\left(V_{2 B}\right)$ induced by a weight one vector $h \in\left(V_{2 B}\right)_{1}$. Since $\psi_{1}=\psi_{2}=-1$ on the weight one subspace, we have $\psi_{i} \rho_{h}=\rho_{-h} \psi_{i}=\rho_{h}^{-1} \psi_{i}$ for $i=0,1$. Then $\psi_{1}=\rho_{h} \psi_{2}=\rho_{h / 2} \rho_{h / 2} \psi_{2}=\rho_{h / 2} \psi_{2} \rho_{h / 2}^{-1}$ so that $\psi_{1}$ and $\psi_{2}$ are conjugate in $\operatorname{Aut}\left(V_{2 B}\right)$. From this we have the desired isomorphism $\rho_{h / 2}:\left(V_{2 B}\right)^{\left\langle\psi_{2}\right\rangle} \stackrel{\sim}{\rightarrow}\left(V_{2 B}\right)^{\left\langle\psi_{1}\right\rangle}$.

Corollary 7.16. There exists $\rho \in \operatorname{Aut}\left(\left(V^{\natural}\right)^{\left\langle\theta_{\xi}\right\rangle}\right)$ such that $V^{\natural}(1,-1)^{\rho} \simeq V^{\natural}\left(\theta_{\xi}^{2}, 1\right)$.

Proof: Since $V^{\natural}(1,1) \subset \rho_{h / 2}\left(V_{2 B}\right)^{\left\langle\psi_{2}\right\rangle} \cap\left(V_{2 B}\right)^{\left\langle\psi_{2}\right\rangle}=\left(V_{2 B}\right)^{\left\langle\psi_{1}\right\rangle} \cap\left(V_{2 B}\right)^{\left\langle\psi_{2}\right\rangle}, \rho_{h / 2}$ keeps $\left(V^{\natural}\right)^{\left\langle\theta_{\xi}\right\rangle}=V^{\natural}(1,1)$ invariant. Thus the restriction of $\rho_{h / 2}$ on $\left(V^{\natural}\right)^{\left\langle\theta_{\xi}\right\rangle}$ is the desired automorphism.

By the classification of irreducible modules over $V_{\Lambda}^{+}$and the fusion rules in Proposition 7.11, the irreducible untwisted $\left(V_{2 B}\right)^{\left\langle\psi_{2}\right\rangle}$-modules are as follows.

$$
\begin{array}{ll}
V_{\Lambda}^{+} \simeq V^{\natural}(1,1) \oplus V^{\natural}\left(\theta_{\xi}^{2}, 1\right), & V_{\Lambda}^{T+} \simeq V^{\natural}\left(\theta_{\xi}, 1\right) \oplus V^{\natural}\left(\theta_{\xi}^{3}, 1\right), \\
V_{\Lambda}^{-} \simeq V^{\natural}(1,-1) \oplus V^{\natural}\left(\theta_{\xi}^{2},-1\right), & V_{\Lambda}^{T-} \simeq V^{\natural}\left(\theta_{\xi},-1\right) \oplus V^{\natural}\left(\theta_{\xi}^{3},-1\right) .
\end{array}
$$


Actually, the isomorphisms above are induced by $\rho \in \operatorname{Aut}\left(\left(V^{\natural}\right)^{\left\langle\theta_{\xi}\right\rangle}\right)$ defined in Corollary 7.16. By the isomorphisms above, the space

$$
V_{4 A}=V^{\natural}(1,1) \oplus V^{\natural}\left(\theta_{\xi}, 1\right) \oplus V^{\natural}\left(\theta_{\xi}^{2}, 1\right) \oplus V^{\natural}\left(\theta_{\xi}^{3}, 1\right)
$$

is a $\mathbb{Z}_{4}$-graded simple current extension of $\left(V^{\natural}\right)^{\left\langle\theta_{\xi}\right\rangle}$ and isomorphic to $V^{\natural}=V_{\Lambda}^{+} \oplus V_{\Lambda}^{T+}$ as a $\left(V^{\natural}\right)^{\left\langle\theta_{\xi}\right\rangle}$-module. Since both $V_{4 A}$ and $V^{\natural}$ are simple current extensions of $\left(V^{\natural}\right)^{\left\langle\theta_{\xi}\right\rangle}$, these two VOA structures are isomorphic. Therefore, we have obtained our main result in this section.

Theorem 7.17. The VOA $V_{4 A}$ obtained by the $4 A$-twisted orbifold construction of $V^{\natural}$ is isomorphic to $V^{\natural}$.

\section{References}

[ATLAS] J.H. Conway, R.T. Curtis, S.P. Norton, R.A. Parker and R.A. Wilson, ATLAS of finite groups, Clarendon Press, Oxford, 1985.

[ABD] T. Abe, G. Buhl and C. Dong, Rationality, regularity, and $\mathrm{C}_{2}$-cofiniteness, Trans. Amer. Math. Soc. 356 (2004), 3391-3402.

[C] J.H. Conway, A simple construction for the Fischer-Griess monster group, Invent. Math. 79 (1985), 513-540.

[D] C. Dong, Representations of the moonshine module vertex operator algebra, in: Mathematical aspects of conformal and topological field theories and quantum groups, Proc. Joint Summer Research Conference, Mount Holyoke, 1992, ed. P. Sally, M. Flato, J. Lepowsky, N. Reshetikhin and G. Zuckerman, Contemporary Math. 175, Amer. Math. Soc., Providence, 1994, 27-36.

[DGH] C. Dong, R.L. Griess and G. Höhn, Framed vertex operator algebras, codes and the moonshine module, Comm. Math. Phys. 193 (1998), 407-448.

[DGL] C. Dong, R.L. Griess and C.H. Lam, Uniqueness results for the moonshine vertex operator algebra, to appear in Amer. J. Math.; math.QA/0506321.

[DL] C. Dong and J. Lepowsky, Generalized vertex algebras and relative vertex operators, Progress in Math. 112, Birkhäuser, Boston, 1993.

[DLM1] C. Dong, H. Li and G. Mason, Some twisted sectors for the moonshine module, Moonshine, the Monster, and related topics (South Hadley, MA, 1994), 25-43, Contemp. Math., 193, Amer. Math. Soc., Providence, RI, 1996. 
[DLM2] C. Dong, H. Li and G. Mason, Twisted representations of vertex operator algebras, Math. Ann. 310 (1998), 571-600.

[DLM3] C. Dong, H. Li and G. Mason, Modular-invariance of trace functions in orbifold theory and generalized moonshine, Comm. Math. Phys. 214 (2000), 1-56.

[DM1] C. Dong and G. Mason, On quantum Galois theory, Duke Math. J. 86 (1997), 305-321.

[DM2] C. Dong and G. Mason, Rational vertex operator algebras and the effective central charge, Internat. Math. Res. Notices 56 (2004), 2989-3008.

[DMZ] C. Dong, G. Mason and Y. Zhu, Discrete series of the Virasoro algebra and the moonshine module, Proc. Symp. Pure. Math., American Math. Soc. 56 II (1994), 295-316.

[FFR] A. J. Feingold, I. B. Frenkel and J. F.X. Ries, Spinor construction of vertex operator algebras, triality, and $E_{8}^{(1)}$, Contemp. Math. 121 (1991).

[FHL] I. Frenkel, Y.-Z. Huang and J. Lepowsky, On axiomatic approaches to vertex operator algebras and modules, Memoirs Amer. Math. Soc. 104, 1993.

[FLM] I.B. Frenkel, J. Lepowsky and A. Meurman, Vertex Operator Algebras and the Monster, Academic Press, New York, 1988.

[FZ] I.B. Frenkel and Y. Zhu, Vertex operator algebras associated to representation of affine and Virasoro algebras, Duke Math. J. 66 (1992), 123-168.

[GKO] P. Goddard, A. Kent and D. Olive, Unitary representations of the Virasoro and super-Virasoro algebras, Comm. Math. Phys. 103 (1986), 105-119.

[Go] Daniel Gorenstein, Finite groups, Harper \& Row, Publishers, New YorkLondon, 1968.

[H1] Y.-Z. Huang, Virasoro vertex operator algebras, (non-meromorphic) operator product expansion and the tensor product theory, J. Algebra 182 (1996), 201234.

[H2] Y.-Z. Huang, A nonmeromorphic extension of the moonshine module vertex operator algebra, in "Moonshine, the Monster and Related Topics, Proc. Joint Summer Research Conference, Mount Holyoke, 1994" (C. Dong and G. Mason, Eds.), Contemporary Math., pp. 123-148, Amer. Math. Soc., Providence, RI, 1996. 
[H3] Y.-Z. Huang, Differential equations and intertwining operators, Comm. Contemp. Math. 7 (2005), 375-400.

[HL] Y.-Z. Huang and J. Lepowsky, A theory of tensor products for module categories for a vertex operator algebra, III, J. Pure Appl. Algebra. 100 (1995), 141-171.

[L1] C.H. Lam, Twisted representations of code vertex operator algebras, J. Algebra 217 (1999), 275-299.

[L2] C.H. Lam, Some twisted module for framed vertex operator algebras, J. Algebra 231 (2000), 331-341.

[L3] C.H. Lam, Induced modules for orbifold vertex operator algebras, J. Math. Soc. Japan 53 (2001), 541-557.

[LSY] C.H. Lam, S. Sakuma and H. Yamauchi, Ising vectors and automorphism groups of commutant subalgebras related to root systems, to appear in Math. Z.

[LY] C.H. Lam and H. Yamauchi, A characterization of the Moonshine vertex operator algebra by means of Virasoro frames, preprint; math.QA/0609718.

[LYY1] C.H. Lam, H. Yamada and H. Yamauchi, Vertex operator algebras, extended $E_{8}$-diagram, and McKay's observation on the Monster simple group, to appear in Trans. Amer. Math. Soc.

[LYY2] C.H. Lam, H. Yamada and H. Yamauchi, McKay's observation and vertex operator algebras generated by two conformal vectors of central charge $1 / 2$, Internat. Math. Res. Papers 3 (2005), 117-181.

[Li1] H. Li, Symmetric invariant bilinear forms on vertex operator algebras, J. Pure Appl. Algebra 96 (1994), 279-297.

[Li2] H. Li, Local systems of vertex operators, vertex superalgebras and modules, $J$. Pure Appl. Algebra 109 (1996), 143-195.

[LiX] H. Li and X. Xu, A characterization of vertex algebras associated to even lattices, J. Algebra 173 (1995), 253-270.

[McS] F.J. MacWilliams and N.J.A Sloane, The theory of error-correcting codes, North-Holland, 1998.

[McST] F.J. MacWilliams, N.J.A Sloane and J.G. Thompson, Good self-dual codes exist, Discrete Mathematics 3 (1972), 153-162. 
[M1] M. Miyamoto, Griess algebras and conformal vectors in vertex operator algebras, J. Algebra 179 (1996), 528-548.

[M2] M. Miyamoto, Representation theory of code vertex operator algebras, J. Algebra 201 (1998), 115-150.

[M3] M. Miyamoto, A new construction of the moonshine vertex operator algebra over the real number field, Ann. of Math 159 (2004), 535-596.

[R] M. Rosellen, A course in Vertex Algebra, math.QA/0607270.

[SY] S. Sakuma and H. Yamauchi, Vertex operator algebra with two Miyamoto involutions generating $S_{3}$, J. Algebra 267 (2003), 272-297.

[Sh] H. Shimakura, Automorphism group of the vertex operator algebra $V_{L}^{+}$for an even lattice L without roots, J. Algebra 280 (2004), 29-57.

[Y1] H. Yamauchi, Module categories of simple current extensions of vertex operator algebras, J. Pure Appl. Algebra 189 (2004), 315-328.

[Y2] H. Yamauchi, A theory of simple current extensions of vertex operator algebras and applications to the moonshine vertex operator algebra, Ph.D. thesis, University of Tsukuba, 2004.; available on the author's web site:

http://www.ms.u-tokyo.ac.jp/ `yamauchi

[Y3] H. Yamauchi, 2A-orbifold construction and the baby-monster vertex operator superalgebra, J. Algebra 284 (2005), 645-668.

[Z] Y.Zhu, Modular invariance of characters of vertex operator algebras, J. Amer. Math. Soc. 9 (1996), 237-302. 